\title{
Umbral moonshine and the Niemeier lattices
}

\author{
Miranda CN Cheng ${ }^{1 *}$, John FR Duncan² and Jeffrey A Harvey ${ }^{3}$
}

\footnotetext{
*Correspondence: mcheng@uva.nl

1 Korteweg-de Vries Institute of Mathematics, University of

Amsterdam, the Netherlands Full list of author information is available at the end of the article
}

\begin{abstract}
In this paper, we relate umbral moonshine to the Niemeier lattices - the 23 even unimodular positive-definite lattices of rank 24 with non-trivial root systems. To each Niemeier lattice, we attach a finite group by considering a naturally defined quotient of the lattice automorphism group, and for each conjugacy class of each of these groups, we identify a vector-valued mock modular form whose components coincide with mock theta functions of Ramanujan in many cases. This leads to the umbral moonshine conjecture, stating that an infinite-dimensional module is assigned to each of the Niemeier lattices in such a way that the associated graded trace functions are mock modular forms of a distinguished nature. These constructions and conjectures extend those of our earlier paper and in particular include the Mathieu moonshine observed by Eguchi, Ooguri and Tachikawa as a special case. Our analysis also highlights a correspondence between genus zero groups and Niemeier lattices. As a part of this relation, we recognise the Coxeter numbers of Niemeier root systems with a type $\mathrm{A}$ component as exactly those levels for which the corresponding classical modular curve has genus zero.
\end{abstract}

AMS subject classification: 11F22; 11F37; 11F46; 11F50; 20C34; 20C35

Keywords: Mock modular form; Niemeier lattice; Umbral moonshine

\section{Background}

In this paper, we relate umbral moonshine to the Niemeier lattices. This relation associates one case of umbral moonshine to each of the 23 Niemeier lattices and in particular constitutes an extension of our previous work [1], incorporating 17 new instances. Moreover, this prescription displays interesting connections to certain interesting genus zero groups (subgroups $\Gamma<S L_{2}(\mathbb{R})$ that define a genus zero quotient of the upper-half plane) and extended Dynkin diagrams via McKay's correspondence.

To explain this moonshine relation, let us first recall what Niemeier lattices are. In 1973, Niemeier classified the even unimodular positive-definite lattices of rank 24 [2]. There are 24 of them, including the so-called Leech lattice discovered almost a decade earlier in the context of optimal lattice sphere packing in 24 dimensions [3]. Niemeier proved that the Leech lattice is the unique even, unimodular and positive-definite lattice of rank 24 with no root vectors (lattice vectors with norm-square 2), while the other 23 even unimodular rank 24-dimensional lattices all have root systems of the full rank 24. Moreover, these 23 lattices are uniquely labelled by their root systems, which are in turn uniquely specified by the following two conditions: first, they are unions of simply laced root systems with the same Coxeter numbers; second, the total rank is 24.

(c) 2014 Cheng et al.; licensee Springer. This is an Open Access article distributed under the terms of the Creative Commons

Attribution License (http://creativecommons.org/licenses/by/4.0), which permits unrestricted use, distribution, and reproduction in any medium, provided the original work is properly credited. 
We will refer to these 23 root systems as the Niemeier root systems and the 23 corresponding lattices as the Niemeier lattices. In this paper, we associate a finite group and a set of vector-valued mock modular forms to each of these 23 Niemeier lattices. The main result of the present paper is then the umbral moonshine conjecture relating the two.

To understand this statement, let us recall what one means by moonshine. This term was first introduced in mathematics to describe the remarkable monstrous moonshine phenomenon. The study of monstrous moonshine was initiated by John McKay's observation that $196,883+1=196,884$, where the summands on the left are degrees of irreducible representations of the Fischer-Griess monster $\mathbb{M}$ and the number on the right is the coefficient of $q$ in the Fourier expansion of the normalised elliptic modular invariant

$$
J(\tau)=\sum_{m \geq-1} a(m) q^{m}=q^{-1}+196,884 q+21,493,760 q^{2}+\cdots,
$$

where we write $q=e^{2 \pi i \tau}$. Following Thompson's idea [4] that $J(\tau)$ should be the graded dimension of an infinite-dimensional module for $\mathbb{M}$, this observation was later expanded into the full monstrous moonshine conjecture by Conway and Norton [5], conjecturing that the graded character $T_{g}(\tau)$ attached to the monster module

$$
V=\bigoplus_{m \geq-1} V_{m}
$$

and $g \in \mathbb{M}$ should be a principal modulus for a certain genus zero group $\Gamma_{g}<S L_{2}(\mathbb{R})$. (When a discrete group $\Gamma<S L_{2}(\mathbb{R})$ has the property that $\Gamma \backslash \mathbb{H}$ is isomorphic to the Riemann sphere minus finitely many points, there exists a holomorphic function $f$ on $\mathbb{H}$ that generates the field of $\Gamma$-invariant functions on $\mathbb{H}$. Such a function $f$ is called a principal modulus, or Hauptmodul, for $\Gamma$ ). We refer to [6] or the introduction of [1] for a more detailed account of monstrous moonshine.

In 2010, the study of a new type of moonshine was triggered by an observation of Eguchi, Ooguri and Tachikawa, which constituted an analogue of McKay's observation in monstrous moonshine. In the work of Eguchi and Taormina and Eguchi et al. in the 1980s [7-9], these authors encountered a $q$-series

$$
H^{(2)}(\tau)=2 q^{-1 / 8}\left(-1+45 q+231 q^{2}+770 q^{3}+2277 q^{4}+\cdots\right)
$$

in the decomposition of the elliptic genus of a $K 3$ surface into irreducible characters of the $\mathcal{N}=4$ superconformal algebra. It was later understood by Eguchi and Hikami [10] that the above $q$-series is a mock modular form. See the 'Automorphic forms' section for the definition of mock modular forms. Subsequently, the coincidence between the numbers 45, 231, 770, 2277, .. and the dimensions of irreducible representations of $M_{24}$ was pointed out in [11]. This observation was later extended into a Mathieu moonshine conjecture in [12-15] by providing the corresponding twisted characters and the mock modular forms $H_{g}^{(2)}$, and was moreover related in a more general context to the K3-compactification of superstring theory in [12]. Very recently, the existence of an infinite-dimensional $M_{24}$-module underlying the mock modular form (3) and those constructed in [12-15] was shown by T. Gannon [16], although the nature of this $M_{24}$-module 
remains mysterious. See [17] and [18] for a review of this $M_{24}$-mock modular relation, and see [19-23] for recent developments in this direction.

Meanwhile, it was found that Mathieu moonshine is but one example of a more general phenomenon, umbral moonshine. In [1], we associated a finite group $G^{(\ell)}$ and a vectorvalued mock modular form $H_{g}^{(\ell)}=\left(H_{g, r}^{(\ell)}\right)$ with $(\ell-1)$-components for every conjugacy class $[g]$ of $G^{(\ell)}$ to each of the six positive integers $\ell$ such that $\ell-1$ divides 12 , and conjectured that there exists an infinite-dimensional $G^{(\ell)}$-module, the umbral module, with the property that its graded character coincides with the mock modular form $H_{g}^{(\ell)}$ for every conjugacy class $[g] \subset G^{(\ell)}$.

Despite the discovery of this more general framework of umbral moonshine, encompassing Mathieu moonshine as a special case and displaying various beautiful properties, many questions remained unanswered. For example, why these specific umbral groups $G^{(\ell)}$ ? Why are they labelled by divisors of the number 12 ? What is the structure underlying all these instances of moonshine?

In the present paper, we provide partial answers to the above questions. We present evidence that there exists an instance of umbral moonshine naturally associated to each of the 23 Niemeier lattices. As a Niemeier lattice is uniquely determined by its root system $X$, in the main text, we shall use $X$ (or equivalently the corresponding lambency; see Tables 1 and 2) to label the instances of umbral moonshine. In particular, we construct in each instance an umbral group $G^{X}$ as the quotient of the automorphism group of the corresponding Niemeier lattice $L^{X}$ by the normal subgroup generated by refections in root vectors. This property gives a uniform construction as well as a concrete understanding of the umbral groups.

Similarly, we provide a prescription that attaches to each of the 23 Niemeier lattices a distinguished vector-valued modular form - the umbral mock modular form $H^{X}$ which conjecturally encodes the dimensions of the homogeneous subspaces of the

Table 1 Niemeier root systems and principal moduli

\begin{tabular}{cccccccc}
\hline$X$ & $A_{1}^{24}$ & $A_{2}^{12}$ & $A_{3}^{8}$ & $A_{4}^{6}$ & $A_{5}^{4} D_{4}$ & $A_{6}^{4}$ & $A_{7}^{2} D_{5}^{2}$ \\
\hline$\ell$ & 2 & 3 & 4 & 5 & 6 & 7 & 8 \\
\hline$\pi^{X}$ & $\frac{2^{24}}{1^{24}}$ & $\frac{3^{12}}{1^{12}}$ & $\frac{4^{8}}{1^{8}}$ & $\frac{5^{6}}{1^{6}}$ & $\frac{2^{1} 5^{5}}{1^{5} 3^{1}}$ & $\frac{7^{4}}{1^{4}}$ & $\frac{2^{2} 4^{4}}{1^{4} 4^{2}}$ \\
\hline$\Gamma^{X}$ & $2 B$ & $3 B$ & $4 C$ & $5 B$ & $6 E$ & $7 B$ & $8 E$ \\
$X$ & $A_{8}^{3}$ & $A_{9}^{2} D_{6}$ & $A_{11} D_{7} E_{6}$ & $A_{12}^{2}$ & $A_{15} D_{9}$ & $A_{17} E_{7}$ & $A_{24}$ \\
\hline$\ell$ & 9 & 10 & 12 & 13 & 16 & 18 & 25 \\
\hline$\pi^{X}$ & $\frac{9^{3}}{1^{3}}$ & $\frac{2^{1} 10^{3}}{1^{3} 5^{1}}$ & $\frac{2^{2} 3^{1} 12^{3}}{1^{3} 4^{1} 6^{2}}$ & $\frac{13^{2}}{1^{2}}$ & $\frac{2^{1} 16^{2}}{1^{2} 8^{1}}$ & $\frac{2^{1} 3^{1} 18^{2}}{1^{2} 6^{1} 9^{1}}$ & $\frac{25^{1}}{11}$ \\
\hline$\Gamma^{X}$ & $9 B$ & $10 E$ & 121 & $13 B$ & $16 B$ & $18 D$ & $(25 Z)$ \\
$X$ & $D_{4}^{6}$ & $D_{6}^{4}$ & $D_{8}^{3}$ & $D_{10} E_{7}^{2}$ & $D_{12}^{2}$ & $D_{16} E_{8}$ & $D_{24}$ \\
\hline$\ell$ & $6+3$ & $10+5$ & $14+7$ & $18+9$ & $22+11$ & $30+15$ & $46+23$ \\
\hline$\pi^{X}$ & $\frac{2^{6} 6^{6}}{1^{6} 3^{6}}$ & $\frac{2^{4} 10^{4}}{1^{4} 5^{4}}$ & $\frac{2^{3} 14^{3}}{1^{3} 7^{3}}$ & $\frac{2^{3} 3^{2} 18^{3}}{1^{3} 6^{2} 9^{3}}$ & $\frac{2^{2} 22^{2}}{1^{2} 11^{2}}$ & $\frac{2^{2} 3^{1} 5^{1} 30^{2}}{1^{2} 6^{1} 10^{1} 15^{2}}$ & $\frac{2^{1} 46^{1}}{1^{1} 23^{1}}$ \\
\hline$\Gamma^{X}$ & $6 C$ & $10 B$ & $14 B$ & $18 C$ & $22 B$ & $30 G$ & $46 A B$
\end{tabular}

\begin{tabular}{ccc}
$X$ & $E_{6}^{4}$ & $E_{8}^{3}$ \\
\hline$\ell$ & $12+4$ & $30+6,10,15$ \\
\hline$\pi^{X}$ & $\frac{2^{4} 3^{4} 12^{4}}{1^{4} 4^{4} 6^{4}}$ & $\frac{2^{3} 3^{3} 5^{3} 30^{3}}{1^{3} 6^{3} 10^{3} 15^{3}}$ \\
\hline$\Gamma^{X}$ & $12 B$ & $30 A$
\end{tabular}


Table 2 Umbral groups

\begin{tabular}{|c|c|c|c|c|c|c|c|}
\hline$x$ & $A_{1}^{24}$ & $A_{2}^{12}$ & $A_{3}^{8}$ & $A_{4}^{6}$ & $A_{5}^{4} D_{4}$ & $A_{6}^{4}$ & $A_{7}^{2} D_{5}^{2}$ \\
\hline$\ell$ & 2 & 3 & 4 & 5 & 6 & 7 & 8 \\
\hline$G^{X}$ & $M_{24}$ & 2. $M_{12}$ & 2. $A G L_{3}(2)$ & $G L_{2}(5) / 2$ & $G L_{2}(3)$ & $S L_{2}(3)$ & $\mathrm{Dih}_{4}$ \\
\hline $\bar{G}^{x}$ & $M_{24}$ & $M_{12}$ & $A G L_{3}(2)$ & $P G L_{2}(5)$ & $P G L_{2}$ (3) & $P S L_{2}(3)$ & $2^{2}$ \\
\hline$x$ & $A_{8}^{3}$ & $A_{9}^{2} D_{6}$ & $A_{11} D_{7} E_{6}$ & $A_{12}^{2}$ & $A_{15} D_{9}$ & $A_{17} E_{7}$ & $A_{24}$ \\
\hline$\ell$ & 9 & 10 & 12 & 13 & 16 & 18 & 25 \\
\hline$G^{X}$ & Dih $_{6}$ & 4 & 2 & 4 & 2 & 2 & 2 \\
\hline $\bar{G}^{x}$ & $\mathrm{Sym}_{3}$ & 2 & 1 & 2 & 1 & 1 & 1 \\
\hline$x$ & $D_{4}^{6}$ & $D_{6}^{4}$ & $D_{8}^{3}$ & $D_{10} E_{7}^{2}$ & $D_{12}^{2}$ & $D_{16} E_{8}$ & $D_{24}$ \\
\hline$\ell$ & $6+3$ & $10+5$ & $14+7$ & $18+9$ & $22+11$ & $30+15$ & $46+23$ \\
\hline$G^{X}$ & 3.Sym 6 & $\mathrm{Sym}_{4}$ & $\mathrm{Sym}_{3}$ & 2 & 2 & 1 & 1 \\
\hline $\bar{G}^{x}$ & Sym$_{6}$ & $\mathrm{Sym}_{4}$ & $\mathrm{Sym}_{3}$ & 2 & 2 & 1 & 1 \\
\hline$x$ & $E_{6}^{4}$ & $E_{8}^{3}$ & & & & & \\
\hline$\ell$ & $12+4$ & $30+6,10,15$ & & & & & \\
\hline$G^{x}$ & $G L_{2}(3)$ & $\mathrm{Sym}_{3}$ & & & & & \\
\hline $\bar{G}^{x}$ & $P G L_{2}$ (3) & $\mathrm{Sym}_{3}$ & & & & & \\
\hline
\end{tabular}

corresponding umbral module. The Niemeier lattice uniquely specifies the shadow of the mock modular form through a map which associates a unary theta series of a specific type to each of the irreducible simply laced ADE root systems, as well as unions of such root systems where all the irreducible components have the same Coxeter number. As will be explained in the 'The Eichler-Zagier operators and an ADE classification' section, this map bears a strong resemblance to the ADE classification by Cappelli et al. of modular invariant combinations of the characters of the $\widehat{A}_{1}$ affine Lie algebra [24]. When applied to the Niemeier root systems, we dictate the resulting unary theta series to be the shadow of the corresponding umbral mock modular form. Together with the natural requirement that $H^{X}$ satisfies an optimal growth condition, the specification of the shadow uniquely fixes the desired umbral form ( $c f$. Theorem 2 and Corollary 2).

By associating a case of umbral moonshine to each Niemeier lattice, we extend our earlier work on umbral moonshine to include 17 more instances. In fact, the six instances discussed in the earlier paper, labelled by the 6 divisors of 12, correspond to pure A-type Niemeier root systems containing only A-type irreducible components. There are eight pure A-type Niemeier root systems, one for each divisor $\ell-1$ of 24 , and they are given simply as the union of $\frac{24}{\ell-1}$ copies of $A_{\ell-1}$. This new proposal relating Niemeier lattices and umbral moonshine can be regarded as a completion of our earlier work [1], in that it includes Niemeier root systems with D- or/and E-components and sheds important light on the underlying structure of umbral moonshine.

More properties of umbral moonshine revealing themselves as new instances are included and as the structure of umbral moonshine is examined in light of the connection to Niemeier lattices. Recall that in [1], we observed a connection between the (extended) Dynkin diagrams and some of the groups $G^{(\ell)}$ via McKay's correspondence for subgroups of $S U(2)$. In the present paper, we observe that the same holds for many of the new instances of umbral moonshine, and the result presents itself as a natural sequence of extended Dynkin diagrams with decreasing rank, starting with $\widehat{E}_{8}$ and ending with $\widehat{A}_{1}$. Moreover, we observe an interesting relation between umbral moonshine and the genus 
zero groups $\Gamma<S L_{2}(\mathbb{R})$ through the shadows of the former and the principal moduli for the latter. As will be discussed in 'Genus zero groups' section and 'From Niemeier lattices to principal moduli' section, our construction attaches a principal modulus for a genus zero group to each Niemeier lattice. In particular, we recognise the Coxeter numbers of the root systems with an A-type component as exactly those levels for which the corresponding classical modular curve has genus zero.

The outline of this paper is as follows. In the 'Groups' section, we give some background on Niemeier lattices, define the umbral finite groups and discuss the mysterious relation to extended ADE diagrams and genus zero quotients of the upper-half plane. In the 'Automorphic forms' section, we introduce various automorphic objects that play a role in umbral moonshine, including (mock) modular forms and Jacobi forms of the weak, meromorphic and mock type. For later use, we also introduce the Eichler-Zagier (or Atkin-Lehner) map on Jacobi forms, and an ADE classification of such maps. In the 'The umbral mock modular forms' section, we focus on the umbral mock modular forms, which are conjecturally the generating functions of the dimensions of the homogeneous subspaces of the umbral modules. In the 'The umbral mock modular forms' and 'The umbral McKay-Thompson series' sections, we give explicit formulas for these umbral mock modular forms as well as most of the umbral McKay-Thompson series. This is achieved partially with the help of multiplicative relations, relating McKay-Thompson series in different instances of umbral moonshine corresponding to Niemeier lattices with one Coxeter number being the multiple of the nother. In the 'Conjectures' section, we present the main results of the paper, which are the umbral moonshine conjectures relating the umbral groups and umbral mock modular forms, and a counterpart for umbral moonshine of the genus zero property of monstrous moonshine. We also observe certain discriminant properties relating the exponents of the powers of $q$ in the mock modular forms and the imaginary quadratic number fields over which the homogeneous submodules of the umbral modules are defined, extending the discriminant properties observed in [1]. Finally, we present some conclusions and discussions in the 'Conclusions and discussion' section.

To provide the data and evidence in support of our conjectures, this paper also contains two Additional files. In Appendix 1, we describe some modular forms and Jacobi forms which are utilised in the paper. In Appendix 2, we present tables of irreducible characters as well as the characters of certain naturally defined (signed) permutation representations of the 23 umbral groups. In Additional file 1, we provide the first few dozen coefficients of all the umbral McKay-Thompson series. In Additional file 2, using the tables in Appendix 2 and 1, we explicitly present decompositions into irreducible representations for the first 10 or so homogeneous subspaces of the umbral modules for all instances of umbral moonshine.

\section{Notation}

We conclude this introduction with a guide to the most important and frequently used notation, and indications as to where the relevant definitions can be found.

$X \quad$ A root system (cf. 'Root systems' section). Usually, $X$ is a union of irreducible simply laced root systems with the same Coxeter number; for example, a Niemeier root system (cf. 'Lattices' section) 
$m$ The Coxeter number of an irreducible root system $X$ (cf. 'Root systems' section), or the Coxeter number of any irreducible component of $X$ when all such numbers coincide

$r \quad$ The rank of a root system $X$ (cf. 'Root systems' section)

$\pi^{X} \quad$ The (formal) product of Frame shapes of Coxeter elements of irreducible components of a root system $X$ (cf. 'Root systems' section)

$W^{X} \quad$ The Weyl group of a root system $X$ (cf. 'Root systems' section)

$L^{X} \quad$ The Niemeier lattice attached to the Niemeier root system $X$ (cf. 'Lattices' section)

$X_{A} \quad$ The union of irreducible components of type A in a Niemeier root system $X$ (cf. 'Lattices' section). Similarly for $X_{D}$ and $X_{E}$

$d_{A}^{X} \quad$ The number of irreducible components of type $\mathrm{A}$ in the root system $X$ (cf. 'Lattices' section). Similarly for $d_{D}^{X}$ and $d_{E}^{X}$

$d^{X} \quad$ The total number of irreducible components of the root system $X$ (cf. 'Genus zero groups' section)

$\Gamma^{X} \quad$ The genus zero subgroup of $S L_{2}(\mathbb{R})$ attached to the Niemeier root system $X$ (cf. 'Genus zero groups' section)

$T^{X} \quad$ A certain principal modulus for $\Gamma^{X}$, for $X$ a Niemeier root system (cf. 'Genus zero groups' section)

$f^{X} \quad$ A certain modular form of weight 2 for $\Gamma^{X}$, for $X$ Niemeier root system (cf. 'Genus zero groups' section and 'From Niemeier lattices to principal moduli' section)

$\ell \quad$ A lambency. A symbol that encodes a genus zero group $\Gamma^{X}$ attached to a Niemeier root system $X$, and thereby also $X$ ( $c f$. 'Genus zero groups' section).

$G^{X} \quad$ The umbral group attached to the Niemeier root system $X$ (cf. 'Umbral groups' section). Also denoted $G^{(\ell)}$ for $\ell$ the lambency corresponding to $X$

$\bar{G}^{X} \quad$ A naturally defined quotient of $G^{X}$ (cf. 'Umbral groups' section). Also denoted $\bar{G}^{(\ell)}$

$\tilde{\chi}^{X} \quad$ A twisted Euler character. A certain naturally defined character of $G^{X}$ (cf.' Umbral groups' section). Similarly for $\tilde{\chi}^{X_{A}}, \chi^{X_{A}}, \bar{\chi}^{X_{A}}, \& \mathrm{c}$

$D^{(\ell)} \quad$ The finite subgroup of $S U(2)$ attached to the umbral group $G^{(\ell)}$ for $2<\ell<11$ (cf. 'McKay correspondence' section)

$\Delta^{(\ell)} \quad$ The extended Dynkin diagram of rank $11-\ell$ attached to the umbral group $G^{(\ell)}$ for $\ell$ as above (cf. 'McKay correspondence' section)

$m$ Usually the index of a Jacobi form (cf. 'Jacobi forms' section). Often, this is chosen to coincide with the Coxeter number $m$ of some root system $X$, in which case we write $m$ for both ( $c f$.'The Eichler-Zagier operators and an ADE classification' section)

$\theta_{m} \quad$ The vector-valued function whose components are the standard index $m$ theta functions $\theta_{m, r}$ for $r \in \mathbb{Z} / 2 m \mathbb{Z}$ ( $c f$. 'Jacobi forms' section)

$\Omega^{X} \quad$ The $2 m \times 2 m$ matrix attached to a simply laced root system $X$ with all irreducible components having Coxeter number $m$ ( $c f$. 'The Eichler-Zagier operators and an ADE classification' section)

$\mathcal{W}^{X} \quad$ The Eichler-Zagier operator on Jacobi forms of index $m$ attached to a simply laced root system $X$ with all irreducible components having Coxeter number $m$ (cf. 'The Eichler-Zagier operators and an ADE classification' section)

$\psi^{P} \quad$ The polar part of a meromorphic Jacobi form $\psi$ (cf. 'From Meromorphic Jacobi forms to mock modular forms' section) 
$\psi^{F} \quad$ The finite part of a meromorphic Jacobi form $\psi$ (cf. 'From Meromorphic Jacobi forms to mock modular forms' section)

$\mu_{m, j} \quad$ A generalised Appell-Lerch sum of index m (cf. 'From Meromorphic Jacobi forms to mock modular forms' section). The function $\mu_{1,0}$ is a meromorphic Jacobi form of weight 1 and index 1 with vanishing finite part. More generally, scalar multiples of $\mu_{m, 0}$ arise as polar parts of certain meromorphic Jacobi forms of weight 1 and index $m$

$h \quad$ Usually a vector-valued mock modular form, with components $h_{r}$ for $r \in \mathbb{Z} / 2 m \mathbb{Z}$, obtained from the theta expansion of the finite part of a meromorphic Jacobi form of weight 1 and index $m$ (cf. 'From Meromorphic Jacobi forms to mock modular forms' section)

$S_{m} \quad$ The vector-valued cusp form of weight $3 / 2$ whose components are the unary theta series $S_{m, r}$ for $r \in \mathbb{Z} / 2 m \mathbb{Z}$ (cf. 'From Meromorphic Jacobi forms to mock modular forms' section), related to $\theta_{m}$ by $S_{m, r}(\tau)=\left.\frac{1}{2 \pi i} \partial_{z} \theta_{m, r}(\tau, z)\right|_{z=0}$

$S^{X} \quad$ The vector-valued cusp form of weight $3 / 2$ attached to a simply laced root system $X$ with all irreducible components having the same Coxeter number (cf. 'The umbral shadows' section). An umbral shadow in case $X$ is a Niemeier root system.

$\psi^{X} \quad$ The unique meromorphic Jacobi form of weight 1 and index $m$ satisfying the conditions of Theorem 2, if such a function exists, where $X$ is a simply laced root system for which all irreducible components have Coxeter number $m$ (cf. 'The umbral shadows' section)

$H^{X} \quad$ The unique vector-valued mock modular form with shadow $S^{X}$ whose components furnish the theta expansion of the finite part of $\psi^{X}$, if $\psi^{X}$ exists ( $c f$. 'The umbral shadows' section). An umbral mock modular form in case $X$ is a Niemeier root system ( $c f$. 'From weight 0 Jacobi forms to umbral mock modular forms' section), and in this situation, also denoted $H^{(\ell)}$ for $\ell$ the lambency corresponding to $X$

$\sigma^{X} \quad$ The skew-holomorphic Jacobi cusp form of weight 2 and index $m$ naturally attached to $X$, where $X$ is a simply laced root system for which all irreducible components have Coxeter number $m$ ( $c f$. 'From Niemeier lattices to principal moduli' section)

$H_{g}^{X} \quad$ The umbral McKay-Thompson series attached to $g \in G^{X}$ for $X$ a Niemeier root system ( $c f$. 'The umbral McKay-Thompson series' section). A vector-valued mock modular form of weight $1 / 2$. Also denoted $H_{g}^{(\ell)}$ for $\ell$ the lambency corresponding to $X$

$S_{g}^{X} \quad$ The vector-valued cusp form conjectured to be the shadow of $H_{g}^{X}$, for $g \in G^{X}$ and $X$ a Niemeier root system (cf. 'Shadows' section and 'Modularity' section).

$\Omega_{g}^{X} \quad$ The $2 m \times 2 m$ matrix attached to $g \in G^{X}$ for $X$ a Niemeier root system with Coxeter number $m$ (cf. 'Shadows' section)

$K^{X} \quad$ The umbral module attached to the Niemeier root system $X$. A conjectural $G^{X}$-module with graded-super-characters given by the $H_{g}^{X}$ ( $c f$. 'Modules' section)

$n_{g} \quad$ The order of the image of an element $g \in G^{X}$ in the quotient group $\bar{G}^{X}$ (cf. 'Modularity' section)

$h_{g} \quad$ The unique positive integer such that $n_{g} h_{g}$ is the product of the shortest and longest cycle lengths in the cycle shape $\widetilde{\Pi}_{g}^{X}$ for $g \in G^{X}$ and $X$ a Niemeier root system (cf. 'Modularity' section) 
$\widetilde{\Pi}_{g}^{X} \quad$ The cycle shape attached to $g \in G^{X}$ via the permutation representation of $G^{X}$ with twisted Euler character $\tilde{\chi}^{X}$ (cf. 'Umbral groups' section and 'Modularity' section, 'Euler characters' in Appendix 2). Similarly for $\widetilde{\Pi}_{g}^{X_{A}}, \widetilde{\Pi}_{g}^{X_{D}}, \& \mathrm{c}$

$v_{g}^{X} \quad$ The multiplier system of $H_{g}^{X}$ (cf. 'Modularity' section)

\section{Groups}

\section{Root systems}

In this subsection, we give a brief summary of simply laced root systems and their corresponding Dynkin diagrams. Standard references for this material include $[25,26]$.

Let $V$ be a finite-dimensional vector space of rank $r$ over $\mathbb{R}$ equipped with an inner product $\langle\cdot, \cdot\rangle$. For $v \in V$, define the hyperplane $H_{v}$ to be the set of elements of $V$ orthogonal to $v$ and the reflection in the hyperplane $H_{v}$ to be the linear map $r_{v}: V \rightarrow V$ defined by

$$
r_{v}\left(v^{\prime}\right)=v^{\prime}-2 \frac{\left\langle v, v^{\prime}\right\rangle}{\langle v, v\rangle} v
$$

A finite subset $X \subset V$ of non-zero vectors is a rank $r$ crystallographic root system if

- $X$ spans $V$,

- $r_{\alpha}(X) \in X$ for all $\alpha \in X$,

- $X \cap \mathbb{R} \alpha=\{\alpha,-\alpha\}$ for all $\alpha \in X$,

- $2\langle\alpha, \beta\rangle /\langle\alpha, \alpha\rangle \in \mathbb{Z}$ for all $\alpha, \beta \in X$.

Given a root system $X$, we say that $X$ is irreducible provided that it cannot be partitioned into proper subsets $X=X_{1} \cup X_{2}$ with $\left\langle\alpha_{1}, \alpha_{2}\right\rangle=0$ for all $\alpha_{1} \in X_{1}$ and $\alpha_{2} \in X_{2}$. If $X$ is an irreducible root system, then there are at most two values for the length $\langle\alpha, \alpha\rangle^{1 / 2}$ that occur. If all roots have the same length, then the irreducible root system is called simply laced.

It is possible to choose a subset of roots in $X$ that form a basis of $V$. We define a subset $\Phi=\left\{f_{1}, f_{2}, \cdots, f_{\mathrm{r}}\right\} \subset X$ to be a set of simple roots provided that

- $\Phi$ is a basis for $V$,

- each root $\alpha \in X$ can be written as a linear combination of the $f_{i}$ with integer coefficients

$$
\alpha=\sum_{i=1}^{r} n_{i} f_{i}
$$

and with either all $n_{i} \leq 0$ or all $n_{i} \geq 0$.

Given a choice of simple roots, we define the positive roots of $X$ to be those $\alpha$ for which all $n_{i} \geq 0$ in (5). The negative roots are those for which all $n_{i} \leq 0$. We also define the height of $\alpha$ as in (5) by setting

$$
\operatorname{ht}(\alpha)=\sum_{i=1}^{\mathrm{r}} n_{i} .
$$

To each irreducible root system, we can associate a connected Dynkin diagram as follows. We associate a node to each simple root. The nodes associated to two distinct simple roots $f_{i}, f_{j}$ are then either not connected if $\left\langle f_{i}, f_{j}\right\rangle=0$ or connected by $N_{i j}$ lines with

$$
N_{i j}=\frac{2\left\langle f_{i}, f_{j}\right\rangle}{\left\langle f_{i}, f_{i}\right\rangle} \frac{2\left\langle f_{j}, f_{i}\right\rangle}{\left\langle f_{j}, f_{j}\right\rangle} \in\{1,2,3\} .
$$


The Dynkin diagrams associated to simply laced irreducible root systems all have $N_{i j}=$ $\{0,1\}$ and are of type $A_{n}, D_{n}, E_{6}, E_{7}, E_{8}$ as shown in Figure 1 . Here, the subscript indicates the rank of the associated root system, and in the figure, we choose a specific enumeration of simple roots for later use in the 'Umbral groups' section.

The height function defines a $\mathbb{Z}$-gradation on the set of roots. Every irreducible root system has a unique root $\theta$ of largest height with respect to a given set of simple roots $\Phi=\left\{f_{i}\right\}$ with an expansion

$$
\theta=\sum_{i=1}^{r} a_{i} f_{i}
$$

where the $a_{i}$ are a set of integers known as the Coxeter labels of the root system or Dynkin diagram. If we append the negative of this highest root (the lowest root) to the simple roots of the simply laced root system, we obtain the extended Dynkin diagrams of type $\widehat{A}_{n}, \widehat{D}_{n}, \widehat{E}_{6}, \widehat{E}_{7}, \widehat{E}_{8}$. These are shown in Figure 2, where we indicate the lowest root with a filled-in circle and the simple roots with empty circles.

Given an irreducible root system $X$, its Coxeter number $\mathrm{m}=\mathrm{m}(X)$ is the sum

$$
\mathrm{m}=1+\sum_{i=1}^{\mathrm{r}} a_{i}
$$

An equivalent definition of the Coxeter number may be given in terms of the Weyl group of $X$. The Weyl group $W^{X}$ is the group generated by the reflections $r_{\alpha}$ for $\alpha \in X$. The product $w=r_{1} r_{2} \ldots r_{\mathrm{r}}$ of reflections $r_{i}:=r_{f_{i}}$ in simple roots $f_{i} \in \Phi$ is called a Coxeter element of $W^{X}$ and is uniquely determined up to conjugacy in $W^{X}$, meaning that different choices of simple roots and different orderings of the simple roots chosen lead

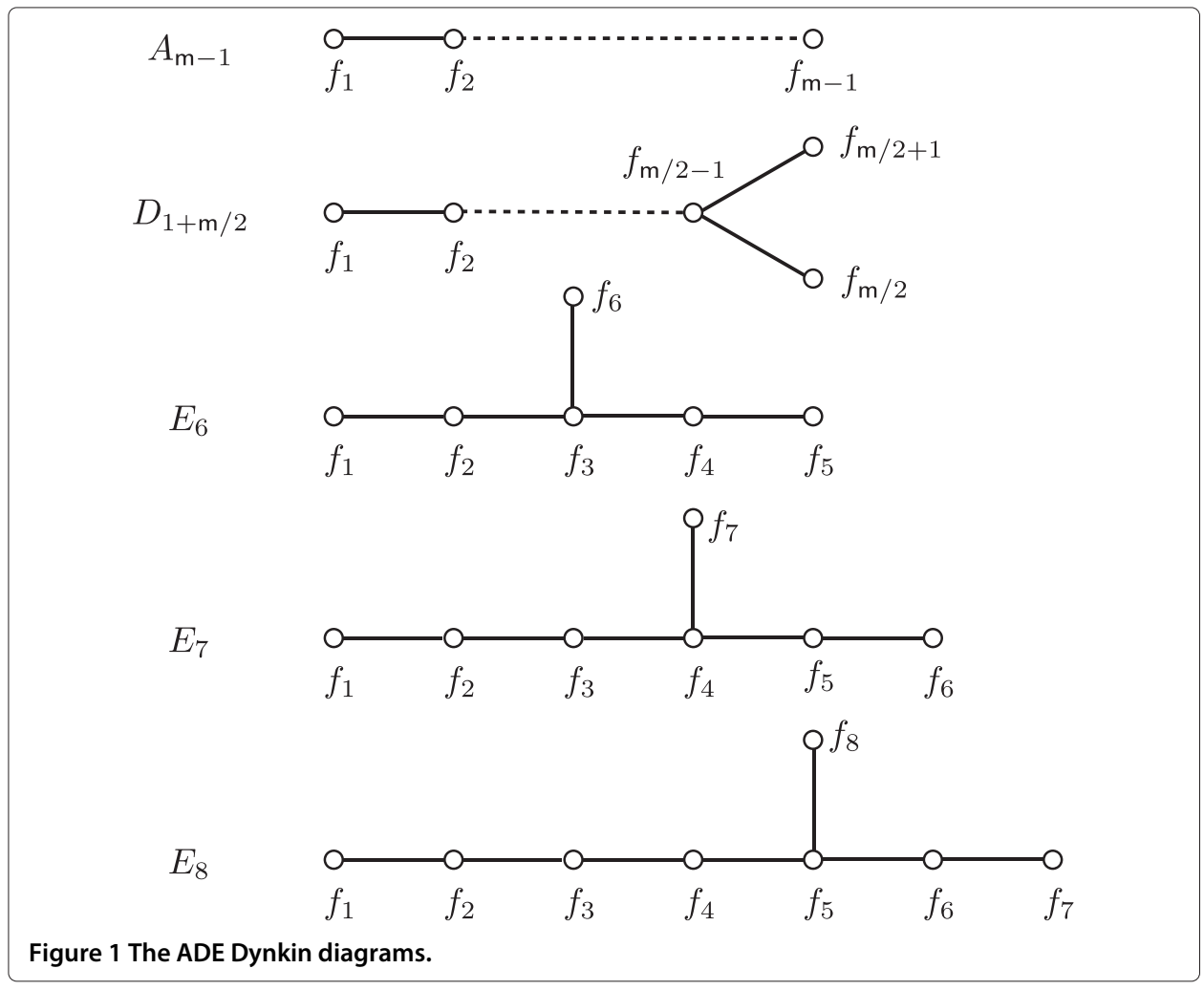




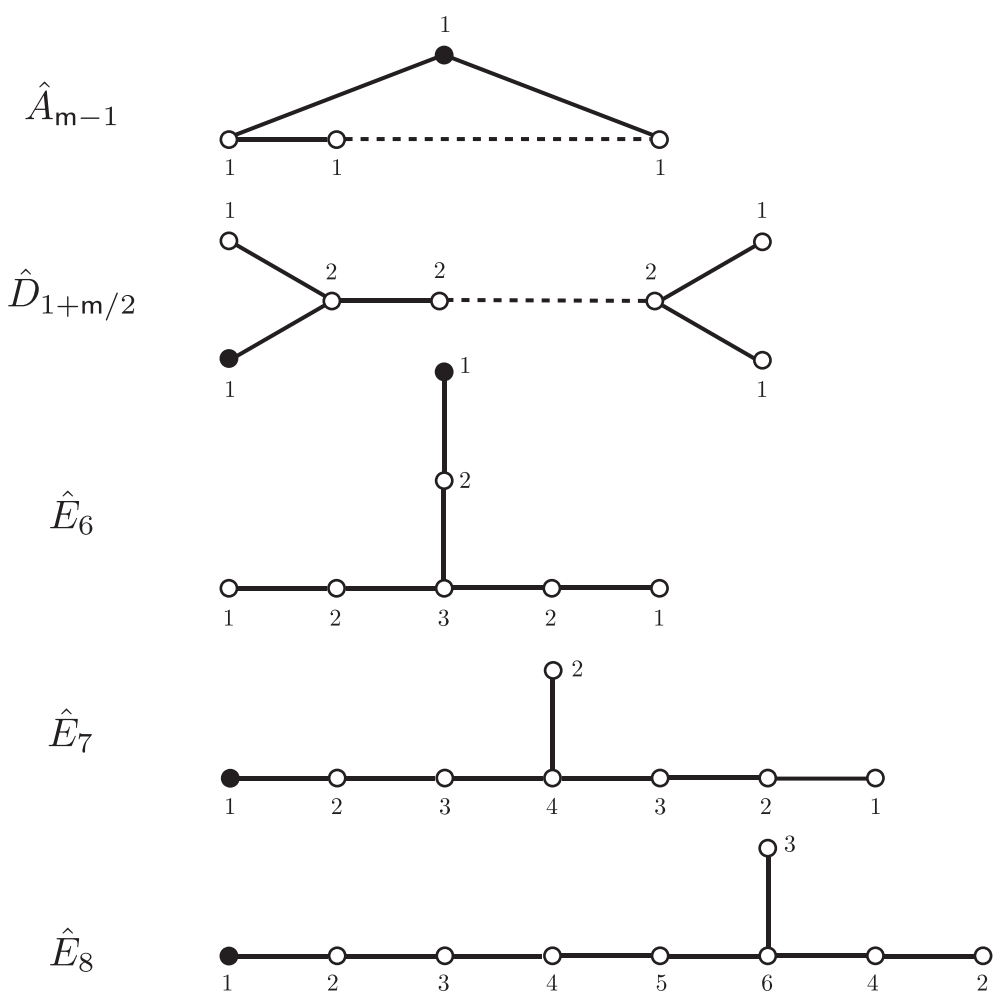

Figure 2 The extended ADE Dynkin diagrams.

to conjugate elements of $W^{X}$. The Coxeter number $m=m(X)$ is then the order of any Coxeter element of $X$.

We obtain a finer invariant of $X$ by considering the eigenvalues of a Coxeter element of $W^{X}$. Say $u_{1}, \ldots, u_{\mathrm{r}}$ are the Coxeter exponents of $X$ if a Coxeter element $w$ has eigenvalues $e^{2 \pi i u_{1} / \mathrm{m}}, \ldots, e^{2 \pi i u_{\mathrm{r}} / \mathrm{m}}$ (counting multiplicity). This data is conveniently recorded using the notion of Frame shape, whereby a formal product $\prod_{i} n_{i}^{k_{i}}$ (with $n_{i}, k_{i} \in \mathbb{Z}$ and $n_{i}>0$ ) serves as a shorthand for the rational polynomial $\prod_{i}\left(x^{n_{i}}-1\right)^{k_{i}}$. For each Coxeter element, there is a Frame shape $\pi^{X}$ - the Coxeter Frame shape of $X$ - such that the corresponding polynomial function coincides with the characteristic polynomial $\prod_{i=1}^{\mathrm{r}}\left(x-e^{2 \pi i u_{i} / \mathrm{m}}\right)$ of $w$. These Frame shapes will play a prominent role in what follows. They are given along with the corresponding Coxeter numbers in Table 3.

Table 3 Coxeter numbers, exponents and frame shapes

\begin{tabular}{|c|c|c|c|c|c|}
\hline & $A_{\mathrm{m}-1}$ & $D_{1+\mathrm{m} / 2}$ & $E_{6}$ & $E_{7}$ & $E_{8}$ \\
\hline $\begin{array}{l}\text { Coxeter } \\
\text { number }\end{array}$ & $\mathrm{m}$ & $\mathrm{m}$ & 12 & 18 & 30 \\
\hline $\begin{array}{l}\text { Coxeter } \\
\text { exponents }\end{array}$ & $1,2,3, \ldots, m-1$ & $\begin{array}{c}1,3,5, \ldots, m-1 \\
m / 2\end{array}$ & $\begin{array}{r}1,4,5 \\
7,8,11\end{array}$ & $\begin{array}{c}1,5,7,9 \\
11,13,17\end{array}$ & $\begin{array}{c}1,7,11,13 \\
17,19,23,29\end{array}$ \\
\hline $\begin{array}{l}\text { Coxeter } \\
\text { frame shapes }\end{array}$ & $\frac{m}{T}$ & $\frac{2 . \mathrm{m}}{1 .(\mathrm{m} / 2)}$ & $\frac{2.3 .12}{1.4 .6}$ & $\frac{2.3 .18}{1.6 .9}$ & $\frac{2.3 .5 .30}{1 \cdot 6 \cdot 10.15}$ \\
\hline
\end{tabular}




\section{Lattices}

A lattice is a free $\mathbb{Z}$-module equipped with a symmetric bilinear form $\langle\cdot, \cdot\rangle$. We say that a lattice $L$ is positive-definite if $\langle\cdot, \cdot\rangle$ induces a positive-definite inner product on the vector space $L_{\mathbb{R}}=L \otimes_{\mathbb{Z}} \mathbb{R}$. Since $L$ is a free $\mathbb{Z}$-module, the natural map $L \rightarrow L_{\mathbb{R}}$ is an embedding and we may identify $L$ with its image in $L_{\mathbb{R}}$. Say that $L$ is integral if we have $\langle\lambda, \mu\rangle \in \mathbb{Z}$ for all $\lambda, \mu \in L$ and say that $L$ is even if we have $\langle\lambda, \lambda\rangle \in 2 \mathbb{Z}$ for each $\lambda \in \Lambda$. (An even lattice is necessarily integral). The dual of $L$ is the lattice $L^{*} \subset L_{\mathbb{R}}$ defined by setting

$$
L^{*}=\left\{\lambda \in L_{\mathbb{R}} \mid\langle\lambda, L\rangle \subset \mathbb{Z}\right\} .
$$

Clearly, if $L$ is integral, then $L^{*}$ contains $L$. In the case that $L^{*}$ coincides with (the image of) $L$ (in $L_{\mathbb{R}}$ ), we say that $L$ is unimodular. For an even lattice $L$, we call $L_{2}=\{\lambda \in L$ | $\langle\lambda, \lambda\rangle=2\}$ the set of roots of $L$.

The Leech lattice is the unique (up to isomorphism) even, unimodular, positive-definite lattice of rank 24 with no roots [27] and is named for its discoverer, John Leech [3,28]. Shortly after Leech's work, the unimodular even positive-definite lattices of rank 24 were classified by Niemeier [2]; we refer to those with non-empty root sets as the Niemeier lattices. There are exactly 23 Niemeier lattices up to isomorphism, and if $L$ is such a lattice, then its isomorphism type is determined by its root set $L_{2}$, which is a union of irreducible simply laced root systems ( $c f$. 'Root systems' section). Say a root system $X$ is a Niemeier root system if it occurs as $L_{2}$ for some Niemeier lattice $L$. The Niemeier root systems are precisely the 23 root systems satisfying the two conditions that first, they are unions of simply laced root systems with the same Coxeter numbers, and second, the total rank is 24. Explicitly, they are

$$
\begin{aligned}
& A_{1}^{24}, A_{2}^{12}, A_{3}^{8}, A_{4}^{6}, A_{6}^{4}, A_{8}^{3}, A_{12}^{2}, A_{24}, A_{5}^{4} D_{4}, A_{7}^{2} D_{5}^{2}, A_{9}^{2} D_{6}, A_{11} D_{7} E_{6}, A_{15} D_{9}, A_{17} E_{7} \\
& D_{4}^{6}, D_{6}^{4}, D_{8}^{3}, D_{10} E_{7}^{2}, D_{12}^{2}, D_{16} E_{8}, D_{24} \\
& E_{6}^{4}, E_{8}^{3} .
\end{aligned}
$$

In (11) we list the Niemeier root systems containing a type $A$ component, in (12) we list the root systems containing a type $D$ component but no type $A$ component, and the remaining two root systems, having only type $E$ components, appear in (13). We will call them the A-type, D-type and the E-type Niemeier root systems, respectively. We say that a Niemeier root system $X$ has Coxeter number $\mathrm{m}$ if $\mathrm{m}$ is the Coxeter number of any simple component of $X$.

Since all the simple components of a Niemeier root system have the same Coxeter number, all the type $A$ components appearing have the same rank, and similarly for components of type $D$ and $E$. So we can write

$$
X=X_{A} X_{D} X_{E}
$$

where $X_{A}=A_{\mathrm{m}-1}^{d_{A}^{X}}$ for some non-negative integer $d_{A}^{X}$ (or $X_{A}=\emptyset$ ), and $\mathrm{m}$ the Coxeter number of $X$, and similarly for $X_{D}$ and $X_{E}$. For example, if $X=A_{7}^{2} D_{5}^{2}$, then $\mathrm{m}=8$, $X_{A}=A_{7}^{2}, X_{D}=D_{5}^{2}, d_{A}^{X}=d_{D}^{X}=2$ and $X_{E}=\emptyset$.

Before finishing this subsection, we will comment on the relation between the Niemeier lattices and the Leech lattice. The covering radius of the Leech lattice is $\sqrt{2}$ according to 
[29], meaning that $R=\sqrt{2}$ is the minimal positive $R$ such that the 24-dimensional vector space $\Lambda_{\mathbb{R}}=\Lambda \otimes_{\mathbb{Z}} \mathbb{R}$ is covered by placing a closed ball of radius $R$ at each point of $\Lambda$,

$$
\sqrt{2}=\sup _{x \in \Lambda_{\mathbb{R}}} \inf _{\lambda \in \Lambda}\|x-\lambda\| .
$$

A point $x \in \Lambda_{\mathbb{R}}$ that realizes the maximum value $\sqrt{2}=\inf _{\lambda \in \Lambda}\|x-\lambda\|$ is called a deep hole of $\Lambda$. Let $x \in \Lambda_{\mathbb{R}}$ be a deep hole and let $V_{x}$ be the set of vertices of $x$,

$$
V_{x}=\{\lambda \in \Lambda \mid\|x-\lambda\|=\sqrt{2}\} .
$$

It is shown in [29] that if $\lambda, \lambda^{\prime} \in V_{x}$ with $\lambda \neq \lambda^{\prime}$, then $\left\|\lambda-\lambda^{\prime}\right\|^{2} \in\{4,6,8\}$. Following [29], define the hole diagram attached to $x$ by joining vertices $\lambda, \lambda^{\prime} \in V_{x}$ with a single edge if $\left\|\lambda-\lambda^{\prime}\right\|^{2}=6$ and by joining them with a double edge if $\left\|\lambda-\lambda^{\prime}\right\|^{2}=8$. The vertices $\lambda$ and $\lambda^{\prime}$ are disjoined in case $\left\|\lambda-\lambda^{\prime}\right\|^{2}=4$. Then, the diagram so obtained is the extended Dynkin diagram corresponding to a Niemeier root system, and all Niemeier root systems arise in this way [29]. Conversely, from each Niemeier lattice, one can obtain a different 'holy' construction of the Leech lattice [30].

\section{Genus zero groups}

In this section, we attach a genus zero subgroup of $S L_{2}(\mathbb{R})$ to each of the 23 Niemeier root systems.

If $\Gamma$ is a discrete subgroup of $S L_{2}(\mathbb{R})$ that is commensurable with the modular group $S L_{2}(\mathbb{Z})$, then its natural action on the boundary $\widehat{\mathbb{R}}=\mathbb{R} \cup\{\mathbf{i} \infty\}$ of the upper-half plane $\mathbb{H}$ restricts to $\widehat{\mathbb{Q}}=\mathbb{Q} \cup\{\mathbf{i} \infty\}$. The orbits of $\Gamma$ on $\widehat{\mathbb{Q}}$ are called the cusps of $\Gamma$, and the quotient space

$$
X_{\Gamma}=\Gamma \backslash \mathbb{H} \cup \widehat{\mathbb{Q}}
$$

is naturally a compact Riemann surface (cf., e.g. in [31], Section 1.5). We adopt the common practice of saying that $\Gamma$ has genus zero in case $X_{\Gamma}$ is a genus zero surface.

For $n$, a positive integer, the Hecke congruence group of level $n$, denoted $\Gamma_{0}(n)$, is defined by setting

$$
\Gamma_{0}(n)=\left\{\left(\begin{array}{cc}
a & b \\
c n & d
\end{array}\right) \mid a, b, c, d \in \mathbb{Z}, a d-b c n=1\right\} .
$$

Say $e$ is an exact divisor of $n$, and write $e \| n$, if $e \mid n$ and $(e, n / e)=1$. According to [5], the normaliser $N\left(\Gamma_{0}(n)\right)$ of $\Gamma_{0}(n)$ in $S L_{2}(\mathbb{R})$ is commensurable with $S L_{2}(\mathbb{Z})$ and admits the description

$$
N\left(\Gamma_{0}(n)\right)=\left\{\frac{1}{\sqrt{e}}\left(\begin{array}{cc}
a e & \frac{b}{h} \\
\frac{c n}{h} & d e
\end{array}\right) \mid a, b, c, d \in \mathbb{Z}, e \| \frac{n}{h}, a d e-b c \frac{n}{e h^{2}}=1\right\}
$$

where $h$ is the largest divisor of 24 such that $h^{2}$ divides $n$. So if $e \| n$, then we obtain a coset $W_{n}(e)$ for $\Gamma_{0}(n)$ in its normaliser by setting

$$
W_{n}(e)=\left\{\frac{1}{\sqrt{e}}\left(\begin{array}{cc}
a e & b \\
c n & d e
\end{array}\right) \mid a, b, c, d \in \mathbb{Z}, e \| n, a d e-b c \frac{n}{e}=1\right\} .
$$

Observe that the product of any two elements of $W_{n}(e)$ lies in $W_{n}(1)=\Gamma_{0}(n)$. More generally, the operation $e * f=e f /(e, f)^{2}$ equips the set of exact divisors of $n$ with a group structure isomorphic to that obtained by multiplication of Atkin-Lehner involutions, $W_{n}(e) W_{n}(f)=W_{n}(e * f)$. So for $S$, a subgroup of the group of exact divisors of $n$, 
we may define a group $\Gamma_{0}(n)+S$, containing and normalizing $\Gamma_{0}(n)$, by taking the union of the Atkin-Lehner cosets $W_{n}(e)$ for $e \in S$. It is traditional [5] to simplify this notation by writing $\Gamma_{0}(n)+e, f, \ldots$ in place of $\Gamma_{0}(n)+\{1, e, f, \ldots\}$. Thus, we have

$$
\Gamma_{0}(n)+S=\left\{\frac{1}{\sqrt{e}}\left(\begin{array}{cc}
a e & b \\
c n & d e
\end{array}\right) \mid a, b, c, d \in \mathbb{Z}, e \in S, a d e-b c \frac{n}{e}=1\right\} .
$$

The positive integers occurring as Coxeter numbers of the A-type Niemeier root systems (cf. (11)) are

$$
2,3,4,5,6,7,8,9,10,12,13,16,18,25 .
$$

Observe that these are exactly the positive integers $n>1$ for which the Hecke congruence group $\Gamma_{0}(n)$ has genus zero ( $c f$., e.g. [32]). The Coxeter numbers of the D-type Niemeier root systems $(c f .(12))$ are

$$
6,10,14,18,22,30,46 \text {, }
$$

and these are exactly the even integers $2 n$ such that the group $\Gamma_{0}(2 n)+n$ has genus zero [32]. We will demonstrate momentarily that the root system $E_{6}^{4}$, having Coxeter number 12 , is naturally attached to the genus zero group $\Gamma_{0}(12)+4$, and $E_{8}^{3}$, having Coxeter number 30 , is naturally attached to the genus zero group $\Gamma_{0}(30)+6,10,15$. As such, we obtain a correspondence between the 23 Niemeier root systems and the genus zero groups of the form

$$
\Gamma_{0}(n), \quad \Gamma_{0}(2 n)+n, \quad \Gamma_{0}(12)+4, \quad \Gamma_{0}(30)+6,10,15 .
$$

Write $\Gamma^{X}$ for the genus zero subgroup of $S L_{2}(\mathbb{R})$ associated in this way to a Niemeier root system $X$. Write $T^{X}$ for the unique principal modulus for $\Gamma^{X}$ that has an expansion

$$
T^{X}=q^{-1}-d^{X}+O(q)
$$

about the infinite cusp, where $d^{X}$ denotes the number of irreducible components of $X$. Then, we may recover $T^{X}$ and hence also $\Gamma^{X}$ directly as follows, from the Coxeter Frame shapes ( $c f$. 'Root systems' section) of the irreducible components of $X$.

Define the Coxeter Frame shape $\pi^{X}$ of an arbitrary root system $X$ to be the product of Coxeter Frame shapes of the irreducible components of $X$. Next, for a Frame shape $\pi=\prod_{i} n_{i}^{k_{i}}$, define the associated eta product $\eta_{\pi}$ by setting

$$
\eta_{\pi}(\tau)=\prod_{i} \eta\left(n_{i} \tau\right)^{k_{i}},
$$

and observe that if $X$ is simply laced and $\pi^{X}$ is the Coxeter Frame shape of $X$, then

$$
\frac{1}{\eta_{\pi^{X}}(\tau)}=q^{-\mathrm{r} / 24}\left(1-d^{X} q+O\left(q^{2}\right)\right)
$$

where $r$ denotes the rank of $X$ and $d^{X}$ is the number of irreducible components. We may also consider the lambda sum $\lambda_{\pi}$ attached to a Frame shape $\pi$, which is the function

$$
\lambda_{\pi}(\tau)=\sum_{i} k_{i} \lambda_{n_{i}}(\tau)
$$

where $\lambda_{n}(\tau)$ is defined in (237) Appendix 1. Observe that if $\pi=\prod_{i} n_{i}^{k_{i}}$ is such that $\sum_{i} k_{i}=0$, then $\lambda_{\pi}=q \frac{d}{d q} \log \eta_{\pi}$.

The Coxeter Frame shapes of the Niemeier root systems are given in Table 1. By inspection, we obtain the following result. 
Proposition 1. If $X$ is a Niemeier root system and $\pi^{X}$ is the Coxeter Frame shape of $X$, then

$$
T^{X}=\frac{1}{\eta_{\pi^{X}}}
$$

is the unique principal modulus for $\Gamma^{X}$ satisfying $T^{X}=q^{-1}-d^{X}+O(q)$ as $\tau \rightarrow i \infty$.

Remark 1. Niemeier's classification of even unimodular positive-definite lattices of rank 24 together with Proposition 1 implies that if $X$ is the root system of an even unimodular positive-definite lattice of rank 24, then the eta product of the Coxeter Frame shape of $X$ is a principal modulus for a genus zero subgroup of $S L_{2}(\mathbb{R})$. It would be desirable to have a conceptual proof of this fact.

The relation between the $T^{X}$ and umbral moonshine will be discussed in 'From Niemeier lattices to principal moduli' section, where the weight two Eisenstein forms

$$
f^{X}=\lambda_{\pi^{X}}
$$

will play a prominent role ( $c f$. Table 4). We have $\sum_{i} k_{i}=0$ when $\pi^{X}=\prod_{i} n_{i}^{k_{i}}$ for every Niemeier root system $X$, so the functions $f^{X}$ and $T^{X}$ are related by

$$
f^{X}=\lambda_{\pi}=q \frac{d}{d q} \log \eta_{\pi}=-q \frac{d}{d q} \log T^{X}
$$

for $\pi=\pi^{X}$.

It is interesting to note that all of the $\Gamma^{X}$, except for $X=A_{24}$, appear in monstrous moonshine as genus zero groups for whom monstrous McKay-Thompson series serve as principal moduli. Indeed, all of the Frame shapes $\pi^{X}$, except for $X=A_{24}$, are Frame shapes of elements of Conway's group $\mathrm{Co}_{0}$, the automorphism group of the Leech lattice (cf. [5], Section 7). We observe that for the cases that $\pi^{X}$ is the Frame shape of an element in $\mathrm{Co}_{0}$, the corresponding centralizer in $\mathrm{Co}_{0}$ typically contains a subgroup isomorphic to $G^{X}$.

We include the ATLAS names [33] (see also [5]) for the monstrous conjugacy classes corresponding to the groups $\Gamma^{X}$ via monstrous moonshine in the rows labelled $\Gamma^{X}$ in Table 1. Extending the notation utilised in [1], we assign lambencies $\ell$ - now symbols rather than integers - to each Niemeier system $X$ according to the prescription of Table 1 . The lambencies then serve to name the groups $\Gamma^{X}$ also, according to the convention that $n$ corresponds to $\Gamma_{0}(n)$, and $12+4$ corresponds to $\Gamma_{0}(12)+4, \&$ c. It will be convenient in what follows to sometimes use $(\ell)$ in place of $X$, writing $G^{(\ell)}, H^{(\ell)}$, \&c., to label the finite groups and mock modular forms associated to the corresponding Niemeier root system.

Table 4 The weight 2 modular forms $f^{X}$

\begin{tabular}{lccc}
\hline $\boldsymbol{X}$ & $\boldsymbol{m}(\boldsymbol{X})$ & $\boldsymbol{\pi}^{\boldsymbol{X}}$ & $\boldsymbol{f}^{\boldsymbol{X}}$ \\
\hline$A_{m-1}$ & $m$ & $\frac{m}{1}$ & $\lambda_{m}$ \\
$D_{m / 2+1}$ & $m$ & $\frac{2 . m}{1 .(m / 2)}$ & $\lambda_{2}+\lambda_{m}-\lambda_{m / 2}$ \\
$E_{6}$ & 12 & $\frac{2.3 .12}{1.4 .6}$ & $\lambda_{2}+\lambda_{3}+\lambda_{12}-\lambda_{4}-\lambda_{6}$ \\
$E_{7}$ & $\frac{2.3 .18}{1.6 .9}$ & $\lambda_{2}+\lambda_{3}+\lambda_{18}-\lambda_{6}-\lambda_{9}$ \\
$E_{8}$ & 18 & $\frac{2.3 .5 .30}{1.6 .10 .15}$ & $\lambda_{2}+\lambda_{3}+\lambda_{5}+\lambda_{30}-\lambda_{6}-\lambda_{10}-\lambda_{15}$ \\
\hline
\end{tabular}




\section{Umbral groups}

Given a Niemeier root system $X$, we may consider the automorphism group of the associated Niemeier lattice $L^{X}$. The reflections in roots of $L^{X}$ generate a normal subgroup of the full automorphism group of $L^{X}$ - the Weyl group of $X$ - which we denote $W^{X}$. We define $G^{X}$ to be the corresponding quotient,

$$
G^{X}=\operatorname{Aut}\left(L^{X}\right) / W^{X} \text {. }
$$

The particular groups $G^{X}$ arising in this way are displayed in Table 2. Observe ${ }^{\text {a }}$ that the group $G^{(\ell)}$ of [1] appears here as $G^{X}$ for $X$ the unique root system with a component $A_{\ell-1}$. In fact, the $G^{(\ell)}$ of [1] are exactly those $G^{X}$ for which $X$ is of the form $X=A_{\ell-1}^{d}$ with even $d$. It will develop that, for every Niemeier root system $X$, the representation theory of $G^{X}$ is intimately related to a set of vector-valued mock modular forms $H_{g}^{X}$, to be introduced in 'The umbral mock modular forms' and 'The umbral McKay-Thompson series' sections.

As mentioned in the 'Genus zero groups' section, it will often be useful to use the lambencies to label the groups and mock modular forms associated to a given Niemeier root system. To this end, we define $G^{(n)}=G^{X}$ in case $\Gamma_{0}(n)$ has genus zero and $X$ is the unique A-type Niemeier root system with Coxeter number $n\left(c f\right.$. (11)). We define $G^{(2 n+n)}=G^{X}$ when $\Gamma_{0}(2 n)+n$ has genus zero and $X$ is the unique D-type Niemeier root system with Coxeter number $2 n\left(c f\right.$. (12)). We write $G^{(12+4)}$ for $G^{X}$ when $X=E_{6}^{4}$, and we write $G^{(30+6,10,15)}$ for $G^{X}$ when $X=E_{8}^{3}$.

Observe that the subgroup $\widehat{W}^{X}<\operatorname{Aut}\left(L^{X}\right)$ consisting of automorphisms of $L^{X}$ that stabilize the irreducible components of $X$ is also normal in $\operatorname{Aut}\left(L^{X}\right)$. Define $\bar{G}^{X}$ to be the corresponding quotient,

$$
\bar{G}^{X}=\operatorname{Aut}\left(L^{X}\right) / \widehat{W}^{X},
$$

so that $\bar{G}^{X}$ is precisely the group of permutations of the irreducible components of $X$ induced by automorphisms of $L^{X}$ and is a quotient of $G^{X}$ (viz., the quotient by $\widehat{W}^{X} / W^{X}$ ) since $W^{X}<\widehat{W}^{X}$. It turns out that $\widehat{W}^{X} / W^{X}$ has order 2 when $X_{A} \neq \emptyset$ or $X=X_{E}=E_{6}^{4}$, has order 3 when $X=X_{D}=D_{4}^{6}$, and is trivial otherwise.

Remark 2. In terms of the notation of [30], we have $W^{X}=G_{0}, \widehat{W}^{X} / W^{X} \simeq G_{1}, \bar{G}^{X} \simeq G_{2}$ and $G^{X} \simeq G_{1} G_{2}$.

The groups $G^{X}$ and $\bar{G}^{X}$ come naturally equipped with various permutation representations. To see, this choose a set $\Phi$ of simple roots for $L^{X}$, meaning a set which is the union of sets of simple roots for each irreducible root sublattice of $L^{X}$. Then, $\Phi$ constitutes a basis for the 24-dimensional space $L_{\mathbb{R}}^{X}$, and for each $g \in G^{X}$, there is a unique element in the pre-image of $g$ under $\operatorname{Aut}\left(L^{X}\right) \rightarrow G^{X}$ that belongs to the subgroup $\operatorname{Aut}\left(L^{X}, \Phi\right)$, consisting of automorphisms of $L^{X}$ that stabilize $\Phi$ as a set (i.e. act as permutations of the irreducible root subsystems followed by permutations - corresponding to Dynkin diagram automorphisms - of simple roots within irreducible root subsystems). Thus, we obtain a section $G^{X} \rightarrow \operatorname{Aut}\left(L^{X}\right)$ of the projection $\operatorname{Aut}\left(L^{X}\right) \rightarrow G^{X}$ whose image is $\operatorname{Aut}\left(L^{X}, \Phi\right)$, and composition with the natural map $\operatorname{Aut}\left(L^{X}, \Phi\right) \rightarrow \operatorname{Sym}_{\Phi}$ defines a permutation representation of $G^{X}$ on $\Phi$. Write $\Phi=\Phi_{A} \cup \Phi_{D} \cup \Phi_{E}$ where $\Phi_{A}$ contains the roots in $\Phi$ belonging to type $A$ components of $X$, and similarly for $\Phi_{D}$ and $\Phi_{E}$. Then, the decomposition $\Phi=\Phi_{A} \cup \Phi_{D} \cup \Phi_{E}$ is stable under $G^{X}$, since $\operatorname{Aut}\left(L^{X}\right)$ cannot mix roots that 
belong to non-isomorphic root systems, so we obtain maps $G^{X} \rightarrow \operatorname{Sym}_{\Phi_{A}}, G^{X} \rightarrow \operatorname{Sym}_{\Phi_{D}}$ and $G^{X} \rightarrow \operatorname{Sym}_{\Phi_{E}}$. Write $g \mapsto \tilde{\chi}_{g}^{X}$ for the character of $G^{X}$ attached to the representation $G^{X} \rightarrow \operatorname{Sym}_{\Phi}$, write $g \mapsto \tilde{\chi}_{g}^{X_{A}}$ for that attached to $G^{X} \rightarrow \operatorname{Sym}_{\Phi_{A}}$, and interpret $\tilde{\chi}^{X_{D}}$ and $\tilde{\chi}^{X_{E}}$ similarly. Observe that $\tilde{\chi}^{X}$ (and hence also $\tilde{\chi}^{X_{A}}, \tilde{\chi}^{X_{D}}$ and $\tilde{\chi}^{X_{E}}$ ) are independent of the choice of $\Phi$. We set $\tilde{\chi}^{X_{A}}=0$ in case $\Phi_{A}$ is empty, and similarly for $\tilde{\chi}^{X_{D}}$ and $\tilde{\chi}^{X_{E}}$. We have

$$
\tilde{\chi}^{X}=\tilde{\chi}^{X_{A}}+\tilde{\chi}^{X_{D}}+\tilde{\chi}^{X_{E}} .
$$

The characters $\tilde{\chi}^{X_{A}}, \&$ c., are naturally decomposed further as follows. Suppose that $X_{A} \neq \emptyset$. Then, $X_{A}=A_{\mathrm{m}-1}^{d_{A}}$ for $d_{A}=d_{A}^{X}(c f .(14))$, and we may write

$$
\Phi_{A}=\left\{f_{j}^{i} \mid 1 \leq i \leq d_{A}, 1 \leq j \leq \mathrm{m}-1\right\}
$$

where the superscript indicates the $A_{\mathrm{m}-1}$ component to which the simple $\operatorname{root} f_{j}^{i}$ belongs, and the inner products between the $f_{j}^{i}$ for varying $j$ are as described by the labeling in Figure 1 (so that $\left\langle f_{j}^{i}, f_{k}^{i}\right\rangle$ is -1 or 0 accordingly as the nodes labelled $f_{j}$ and $f_{k}$ are joined by an edge or not). Then, for fixed $j$, the vectors $\left\{f_{j}^{i}+f_{\mathrm{m}-j}^{i} \mid 1 \leq i \leq d_{A}\right\}$ define a permutation representation of degree $d_{A}$ for $G^{X}$. We denote the corresponding character $g \mapsto \bar{\chi}_{g}^{X_{A}}$ since the isomorphism type of the representation is evidently independent of the choice of $j$. Observe that $\bar{\chi}^{X_{A}}$ is generally not a faithful character since permutations of $\Phi_{A}$ arising from diagram automorphisms, exchanging $f_{j}^{i}$ with $f_{\mathrm{m}-j}^{i}$ for some $i$, act trivially. The vectors $\left\{f_{j}^{i}-f_{\mathrm{m}-j}^{i} \mid 1 \leq i \leq d_{A}\right\}$ also span $G^{X}$-invariant subspaces of $\operatorname{Span}_{\mathbb{R}} \Phi_{A}<L_{\mathbb{R}}^{X}$, with different $j$ in the range $0<j<\mathrm{m} / 2$ furnishing isomorphic (signed permutation) representations; we denote the corresponding character $g \mapsto \chi_{g}^{X_{A}}$. Since the $f_{j}^{i}$ are linearly independent, we can conclude that

$$
\tilde{\chi}^{X_{A}}=\left\lceil\frac{\mathrm{m}-1}{2}\right\rceil \bar{\chi}^{X_{A}}+\left\lfloor\frac{\mathrm{m}-1}{2}\right\rfloor \chi^{X_{A}}
$$

by counting the possibilities for $j$ in each case.

If $\Phi_{D}$ is non-empty, then $\mathrm{m}$ is even and $X_{D}=D_{\mathrm{m} / 2+1}^{d_{D}}$ for $d_{D}=d_{D}^{X}(c f .(14))$. Write now

$$
\Phi_{D}=\left\{f_{j}^{i} \mid 1 \leq i \leq d_{D}, 1 \leq j \leq \mathrm{m} / 2+1\right\}
$$

where, similar to the above, the superscript indicates the $D_{\mathrm{m} / 2+1}$ component to which the simple root $f_{j}^{i}$ belongs, and the inner products between the $f_{j}^{i}$ for varying $j$ are as described in Figure 1. Suppose first that $m>6$. Then, $m / 2+1>4$ and the only non-trivial diagram automorphism of $D_{\mathrm{m} / 2+1}$ has order 2 and interchanges $f_{\mathrm{m} / 2}^{i}$ and $f_{\mathrm{m} / 2+1}^{i}$. So we find that for $1 \leq j<\mathrm{m} / 2$, the sets $\left\{f_{j}^{i} \mid 1 \leq i \leq d_{D}\right\}$ serve as bases for isomorphic permutation representations of degree $d_{D}$ for $G^{X}$, as does $\left\{f_{\mathrm{m} / 2}^{i}+f_{\mathrm{m} / 2+1}^{i} \mid 1 \leq i \leq d_{D}\right\}$; we denote the character of this (i.e. any one of these) permutation representation(s) by $g \mapsto \bar{\chi}_{g}^{X_{D}}$. We define $\chi^{X_{D}}$ to be the (signed permutation) character of the representation spanned by the vectors $\left\{f_{\mathrm{m} / 2}^{i}-f_{\mathrm{m} / 2+1}^{i} \mid 1 \leq i \leq d_{D}\right\}$, and we have

$$
\tilde{\chi}^{X_{D}}=\frac{\mathrm{m}}{2} \bar{\chi}^{X_{D}}+\chi^{X_{D}}
$$

when $X_{D} \neq \varnothing$ and $\mathrm{m}>6$. In case $\mathrm{m}=6$, the group of diagram automorphisms of $D_{\mathrm{m} / 2+1}=D_{4}$ is a copy of $S_{3}$, acting transitively on the sets $\left\{f_{1}^{i}, f_{3}^{i}, f_{4}^{i}\right\}$ (for fixed $i$ ), so we define $\bar{\chi}^{X_{D}}$ to be the character attached to the (permutation) representation of $G^{X}$ spanned by $\left\{f_{2}^{i} \mid 1 \leq i \leq d_{D}\right\}$ (or equivalently, $\left\{f_{1}^{i}+f_{3}^{i}+f_{4}^{i} \mid 1 \leq i \leq d_{D}\right\}$ ) and define 
$\check{\chi}^{X_{D}}$ to be the character of the representation spanned by the vectors $\left\{f_{1}^{i}-f_{3}^{i}, f_{1}^{i}-f_{4}^{i} \mid 1 \leq\right.$ $\left.i \leq d_{D}\right\}$. Evidently,

$$
\tilde{\chi}^{X_{D}}=2 \bar{\chi}^{X_{D}}+\check{\chi}^{X_{D}}
$$

in case $m=6$. In preparation for the 'Shadows' section, where the characters defined here will be used to specify certain vector-valued cusp forms of weight $3 / 2$, we define $\chi_{g}^{X_{D}}=\operatorname{sgn}_{g}^{X_{D}} \bar{\chi}_{g}^{X_{D}}$ for $g \in G^{X}$ when $X=A_{4}^{5} D_{4}$ or $X=D_{4}^{6}$ - the two cases for which $X$ involves $D_{4}$ - where $\operatorname{sgn}_{g}^{X_{D}}= \pm 1$ is the function defined as follows. Write the image of $g \in G^{X}$ in $\operatorname{Sym}_{\Phi_{D}}$ as a product $g_{d} \circ g_{p}$ where $g_{p} \cdot f_{j}^{i}=f_{j}^{\pi(i)}$ for all $j \in\{1,2,3,4\}$, for some permutation $\pi \in \operatorname{Sym}_{d_{D}^{X}}$, and $g_{d} \cdot f_{j}^{i}=f_{\sigma_{i}(j)}^{i}$ for some permutations $\sigma_{i} \in \operatorname{Sym}_{4}$. Then, set $\operatorname{sgn}_{g}^{X_{D}}=\prod_{i=1}^{d_{D}^{X}} \operatorname{sgn}\left(\sigma_{i}\right)$.

If $\Phi_{E}=E_{n}^{d_{E}}$ for $d_{E}=d_{E}^{X}>0$, then we may identify $f_{j}^{i} \in \Phi_{E}$ such that

$$
\Phi_{E}=\left\{f_{j}^{i} \mid 1 \leq i \leq d_{E}, 1 \leq j \leq n\right\}
$$

and, as above, the superscripts enumerate simple components of $X_{E}$ and the subscripts indicate inner products for simple vectors within a component as per Figure 1. Define $\bar{\chi}^{X_{E}}$ to be the character of $G^{X}$ attached to the permutation representation spanned by the set $\left\{f_{3}^{i} \mid 1 \leq i \leq d_{E}\right\}$ (for example). In case $n=6$, write $\chi^{X_{E}}$ for the character of $G^{X}$ attached to the representation afforded by $\operatorname{Span}_{\mathbb{R}}\left\{f_{1}^{i}-f_{5}^{i} \mid 1 \leq i \leq d_{E}\right\}$. We have

$$
\tilde{\chi}^{X_{E}}=n \bar{\chi}^{X_{E}}
$$

when $n \in\{7,8\}$ since for each $1 \leq j \leq n$, the set $\left\{f_{j}^{i} \mid 1 \leq i \leq d_{E}\right\}$ spans a representation with character $\bar{\chi}^{X_{E}}$ in these cases, and

$$
\tilde{\chi}^{X_{E}}=4 \bar{\chi}^{X_{E}}+2 \chi^{X_{E}}
$$

when $n=6$, the invariant subspace with character $2 \chi^{X_{E}}$ being spanned by the vectors $f_{1}^{i}-f_{5}^{i}$ and $f_{2}^{i}-f_{4}^{i}$ for $1 \leq i \leq d_{E}$.

We call the functions $\bar{\chi}_{g}^{X_{A}}, \chi_{g}^{X_{A}}, \bar{\chi}_{g}^{X_{D}}, \chi_{g}^{X_{D}}$, \&c. the twisted Euler characters attached to $G^{X}$. They are given explicitly in the tables of 'Euler characters' in Appendix 2. As mentioned above, we will use them to attach a vector-valued cusp form $S_{g}^{X}$ to each $g \in G^{X}$ for $X$ a Niemeier root system in the 'Shadows' section.

\section{McKay correspondence}

The McKay correspondence [34] relates finite subgroups of $S U(2)$ to the extended Dynkin diagrams of ADE type by associating irreducible representations of the finite groups to nodes of the corresponding diagrams. A beautiful explanation for this can be given in terms of resolutions of simple singularities $\mathbb{C}^{2} / G$ for $G<S U(2)[35,36]$. In Section 3.5 of [1], we observed a curious connection between the umbral groups $G^{(\ell)}$ and certain finite subgroups $D^{(\ell)}<S U(2)$, for the cases $\ell \in\{3,4,5,7\}$, such that the lambency $\ell$ and the rank $r$ of the Dynkin diagram attached to $D^{(\ell)}$ via McKay's correspondence are related by $\ell+r=11$. In this section, we describe an extension of this observation, relating the umbral group $G^{(\ell)}$ to a finite subgroup $D^{(\ell)}<S U(2)$, for each $\ell$ in $\{3,4,5,6,7,8,9,10\}$.

In [1], it was observed that a Dynkin diagram of rank $11-\ell$ may be attached to each $G^{(\ell)}$ for $\ell \in\{3,4,5,7\}$ in the following manner. If $p=(25-\ell) /(\ell-1)$, then $p$ is a prime and there is a unique (up to conjugacy) subgroup $\bar{L}^{(\ell)}<\bar{G}^{(\ell)}$ such that $\bar{L}^{(\ell)}$ is isomorphic to $P S L_{2}(p)$ and acts transitively in the degree $24 /(\ell-1)$ permutation representation of 
$G^{(\ell)}$ defined in ([1], section 3.3). Now $\bar{L}^{(\ell)}$ has a unique (up to isomorphism) subgroup $\bar{D}^{(\ell)}$ of index $p$ in $\bar{L}^{(\ell)}$ - a fact which is peculiar to the particular $p$ arising - and $\bar{D}^{(\ell)}$ is a finite subgroup of $S O(3)$ whose pre-image $D^{(\ell)}$ in $S U(2)$ realises the extended diagram $\Delta^{(\ell)}$ corresponding ( $c f$. Figure 2) to a Dynkin diagram of rank $11-\ell$ via McKay's correspondence. In the present setting, with groups $G^{(\ell)}$ defined for all $\ell$ such that $\Gamma_{0}(\ell)$ has genus zero, and in particular for $3 \leq \ell \leq 10$, it is possible to extend this correspondence as follows.

Since $(25-\ell) /(\ell-1)$ is not an integer for $\ell \in\{6,8,10\}$, we seek a new definition of $p$. Armed with the Niemeier root systems attached to each $G^{(\ell)}$, we set $p=d_{A}-1$ in case $X=X_{A}=A_{\ell-1}^{d_{A}}$ has only A-type components, and set $p=d_{A}$ otherwise. This definition yields values coincident with the former one when $(25-\ell) /(\ell-1)$ is an integer. Next, we seek a subgroup $\bar{L}^{(\ell)}<\bar{G}^{(\ell)}$ acting transitively on the irreducible components of $X_{A}$ and $X_{D}$ that has a unique up to isomorphism index $p$ subgroup $\bar{D}^{(\ell)}$,

$$
\left[\bar{L}^{(\ell)}: \bar{D}^{(\ell)}\right]=p .
$$

Such $\bar{L}^{(\ell)}$ and $\bar{D}^{(\ell)}$ exist for each $3 \leq \ell \leq 10$ and are given explicitly in Table 5 . In the new cases $\ell \in\{6,8,9,10\}$, the groups $\bar{L}^{(\ell)}$ and $\bar{G}^{(\ell)}$ coincide.

The main observation of this section is the following:

For every $3 \leq \ell \leq 10$, the group $\bar{D}^{(\ell)}$ is the image in $S O(3)$ of a finite subgroup $D^{(\ell)}<S U(2)$ that is attached, via McKay's correspondence, to the extended diagram $\Delta^{(\ell)}$ corresponding to a Dynkin diagram of rank $11-\ell$.

The group $D^{(\ell)}$ is even a subgroup of $G^{(\ell)}$ - the pre-image under the natural map $G^{X} \rightarrow \bar{G}^{X}$ - except in the case that $\ell=5$. (We refer to ([1], Section 3.4) for a discussion of this exceptional case). To aid in the reading of Table 5 , we note here the exceptional isomorphisms

$$
\begin{aligned}
& P G L_{2}(5) \simeq \operatorname{Sym}_{5}, P S L_{2}(5) \simeq A l t_{5}, \\
& P G L_{2}(3) \simeq \operatorname{Sym}_{4}, P S L_{2}(3) \simeq \text { Alt }_{4}, \\
& P G L_{2}(2) \simeq P S L_{2}(2) \simeq \text { Sym }_{3} .
\end{aligned}
$$

In [1], we used the common abbreviation $L_{n}(q)$ for $P S L_{n}(q)$.

Recall from Section 3.5 of [1] the following procedure for obtaining a length 8 sequence of Dynkin diagrams. Start with the (finite type) $E_{8}$ Dynkin diagram, being star-shaped with three branches, and construct a sequence of diagrams iteratively by removing the end node from a branch of maximal length at each iteration. In this way, we obtain $E_{8}, E_{7}, E_{6}$, $D_{5}, D_{4}, A_{3}, A_{2}$ and $A_{1}$, and it is striking to observe that our list $\Delta^{(\ell)}$, obtained by applying

Table 5 The McKay correspondence in umbral moonshine

\begin{tabular}{lcccccccc}
\hline $\boldsymbol{X}$ & $\boldsymbol{A}_{\mathbf{2}}^{\mathbf{1 2}}$ & $\boldsymbol{A}_{\mathbf{3}}^{\mathbf{8}}$ & $\boldsymbol{A}_{\mathbf{4}}^{\mathbf{6}}$ & $\boldsymbol{A}_{\mathbf{5}}^{\mathbf{4}} \boldsymbol{D}_{\mathbf{4}}$ & $\boldsymbol{A}_{\mathbf{6}}^{\mathbf{4}}$ & $\boldsymbol{A}_{\mathbf{7}}^{\mathbf{2}} \boldsymbol{D}_{\mathbf{5}}^{\mathbf{2}}$ & $\boldsymbol{A}_{\mathbf{8}}^{\mathbf{3}}$ & $\boldsymbol{A}_{\mathbf{9}}^{\mathbf{2} \boldsymbol{D}_{\mathbf{6}}}$ \\
\hline$\ell$ & 3 & 4 & 5 & 6 & 7 & 8 & 9 & 10 \\
$p$ & 11 & 7 & 5 & 4 & 3 & 2 & 2 & 2 \\
\hline$G^{(\ell)}$ & $2 . M_{12}$ & $2 . A G L_{3}(2)$ & $G L_{2}(5) / 2$ & $G L_{2}(3)$ & $S L_{2}(3)$ & $D i h_{4}$ & $D i h_{6}$ & 4 \\
$D^{(\ell)}$ & $2 . A l t_{5}$ & $2 . S y m_{4}$ & & $D i h_{6}$ & $Q_{8}$ & 4 & 3 & 2 \\
\hline $\bar{G}^{(\ell)}$ & $M_{12}$ & $A G L_{3}(2)$ & $P G L_{2}(5)$ & $P G L_{2}(3)$ & $P S L_{2}(3)$ & $2^{2}$ & $P S L_{2}(2)$ & 2 \\
$\bar{L}^{(\ell)}$ & $P S L_{2}(11)$ & $P S L_{2}(7)$ & $P S L_{2}(5)$ & $P G L_{2}(3)$ & $P S L_{2}(3)$ & $2^{2}$ & $P S L_{2}(2)$ & 2 \\
$\bar{D}^{(\ell)}$ & $A l t_{5}$ & $S y m_{4}$ & $A l t_{4}$ & $S y m_{3}$ & $2^{2}$ & 2 & 3 & 1 \\
\hline$\Delta^{(\ell)}$ & $\widehat{E}_{8}$ & $\widehat{E}_{7}$ & $\widehat{E}_{6}$ & $\widehat{D}_{5}$ & $\widehat{D}_{4}$ & $\widehat{A}_{3}$ & $\widehat{A}_{2}$ & $\widehat{A}_{1}$ \\
\hline
\end{tabular}


the McKay correspondence to distinguished subgroups of the $G^{(\ell)}$, is exactly the sequence obtained from this by replacing (finite type) Dynkin diagrams with their corresponding extended diagrams.

\section{Automorphic forms}

In this section, we discuss modular objects that play a role in the moonshine relation between mock modular forms and finite groups that is the main focus of this paper.

In what follows, we take $\tau$ in the upper half-plane $\mathbb{H}$ and $z \in \mathbb{C}$, and adopt the shorthand notation $e(x)=e^{2 \pi i x}$. We also define $q=e(\tau)$ and $y=e(z)$ and write

$$
\gamma \tau=\frac{a \tau+b}{c \tau+d}, \quad \gamma=\left(\begin{array}{ll}
a & b \\
c & d
\end{array}\right) \in S L_{2}(\mathbb{R})
$$

for the natural action of $S L_{2}(\mathbb{R})$ on $\mathbb{H}$, and write

$$
\gamma(\tau, z)=\left(\frac{a \tau+b}{c \tau+d}, \frac{z}{c \tau+d}\right)
$$

for the action of $S L_{2}(\mathbb{Z})$ on $\mathbb{H} \times \mathbb{C}$. We set

$$
\mathrm{j}(\gamma, \tau)=(c \tau+d)^{-1}
$$

and choose the principal branch of the logarithm (i.e. $x^{s}=|x|^{s} e^{\mathbf{i} \theta s}$ when $x=|x| e^{\mathbf{i} \theta}$ and $-\pi<\theta \leq \pi)$ to define non-integer exponentials.

\section{Mock modular forms}

We briefly recall modular forms, mock modular forms and their vector-valued generalisations.

Let $\Gamma$ be a discrete subgroup of the group $S L_{2}(\mathbb{R})$ that is commensurable with the modular group $S L_{2}(\mathbb{Z})$. For $w \in \frac{1}{2} \mathbb{Z}$, say that a non-zero function $\psi: \Gamma \rightarrow \mathbb{C}$ is a multiplier system for $\Gamma$ with weight $w$ if

$$
\psi\left(\gamma_{1}\right) \psi\left(\gamma_{2}\right) \mathrm{j}\left(\gamma_{1}, \gamma_{2} \tau\right)^{w} \mathbf{j}\left(\gamma_{2}, \tau\right)^{w}=\psi\left(\gamma_{1} \gamma_{2}\right) \mathrm{j}\left(\gamma_{1} \gamma_{2}, \tau\right)^{w}
$$

for all $\gamma_{1}, \gamma_{2} \in \Gamma$. Given such a multiplier system $\psi$ for $\Gamma$, we may define the $(\psi, w)$-action of $\Gamma$ on the space $\mathcal{O}(\mathbb{H})$ of holomorphic functions on the upper half-plane by setting

$$
\left(\left.f\right|_{\psi, w} \gamma\right)(\tau)=f(\gamma \tau) \psi(\gamma) \mathrm{j}(\gamma, \tau)^{w}
$$

for $f \in \mathcal{O}(\mathbb{H})$ and $\gamma \in \Gamma$. We then say that $f \in \mathcal{O}(\mathbb{H})$ is an (unrestricted) modular form with multiplier $\psi$ and weight $w$ for $\Gamma$ in the case that $f$ is invariant for this action, i.e. $\left.f\right|_{\psi, w} \gamma=f$ for all $\gamma \in \Gamma$. We say that an unrestricted modular form $f$ for $\Gamma$ with multiplier $\psi$ and weight $w$ is a weakly holomorphic modular form in case $f$ has at most exponential growth at the cusps of $\Gamma$. We say that $f$ is a modular form if $\left(\left.f\right|_{\widetilde{\psi}, w} \sigma\right)(\tau)$ remains bounded as $\Im(\tau) \rightarrow \infty$ for any $\sigma \in S L_{2}(\mathbb{Z})$, and we say $f$ is a cusp form if $\left(\left.f\right|_{\tilde{\psi}, w} \sigma\right)(\tau) \rightarrow 0$ as $\Im(\tau) \rightarrow \infty$ for any $\sigma \in S L_{2}(\mathbb{Z})$.

Suppose that $\psi$ is a multiplier system for $\Gamma$ with weight $w$ and that $g$ is a modular form for $\Gamma$ with the conjugate multiplier system $\bar{\psi}: \gamma \mapsto \overline{\psi(\gamma)}$ and dual weight $2-w$. Then, we may use $g$ to twist the $(\psi, w)$-action of $\Gamma$ on $\mathcal{O}(\mathbb{H})$ by setting

$$
\left(\left.f\right|_{\psi, w, g} \gamma\right)(\tau)=f(\gamma \tau) \psi(\gamma) \mathrm{j}(\gamma, \tau)^{w}+e\left(\frac{w-1}{4}\right) \int_{-\gamma^{-1} \infty}^{\infty}\left(\tau^{\prime}+\tau\right)^{-w} \overline{g\left(-\bar{\tau}^{\prime}\right)} \mathrm{d} \tau^{\prime} .
$$


With this definition, we say that $f \in \mathcal{O}(\mathbb{H})$ is an (unrestricted) mock modular form with multiplier $\psi$, weight $w$ and shadow $g$ for $\Gamma$ if $f$ is invariant for this action, i.e. $\left.f\right|_{\psi, w, g} \gamma=f$ for all $\gamma \in \Gamma$. We say that an unrestricted mock modular form $f$ for $\Gamma$ with multiplier $\psi$, weight $w$ and shadow $g$ is a weakly holomorphic mock modular form in case $f$ has at most linear exponential growth at the cusps of $\Gamma$. From this point of view, a (weakly holomorphic) modular form is a (weakly holomorphic) mock modular form with vanishing shadow. This notion of mock modular form developed from the Maass form theory due to Bruinier and Funke [37] and from Zwegers' work [38] on Ramanujan's mock theta functions.

In this paper, we will consider the generalisation of the above definition to vector-valued (weakly holomorphic) mock modular forms with $n$ components, where the multiplier $\psi: \Gamma \rightarrow G L_{n}(\mathbb{C})$ is a (projective) representation of $\Gamma$. From the definition (52), it is not hard to see that the multiplier $\psi$ of a (vector-valued) mock modular form is necessarily the inverse of that of its shadow. To avoid clutter, we omit the adjective 'weakly holomorphic' in the rest of the paper when there is no room for confusion.

Following Zwegers [38] and Zagier [39], we define a mock theta function to be a $q$-series $h=\sum_{n} a_{n} q^{n}$ such that for some $\lambda \in \mathbb{Q}$, the assignment $\left.\tau \mapsto q^{\lambda} h\right|_{q=e(\tau)}$ defines a mock modular form of weight $1 / 2$ whose shadow is a unary (i.e. attached to a quadratic form in one variable) theta series of weight $3 / 2$. In the 'Conjectures' section, we conjecture that specific sets of mock theta functions appear as McKay-Thompson series associated to infinite-dimensional modules for the groups $G^{X}$ ( $c f$. 'Umbral groups' section), where $X$ is a Niemeier root system.

\section{Jacobi forms}

We first discuss Jacobi forms following [40]. For every pair of integers $k$ and $m$, we define the $m$-action of the group $\mathbb{Z}^{2}$ and the $(k, m)$-action of the group $S L_{2}(\mathbb{Z})$ on the space of holomorphic functions $\phi: \mathbb{H} \times \mathbb{C} \rightarrow \mathbb{C}$ as

$$
\begin{aligned}
\left(\left.\phi\right|_{m}(\lambda, \mu)\right)(\tau, z) & =e\left(m\left(\lambda^{2} \tau+2 \lambda z\right)\right) \phi(\tau, z+\lambda \tau+\mu) \\
\left(\left.\phi\right|_{k, m} \gamma\right)(\tau, z) & =e\left(-m \frac{c z^{2}}{c \tau+d}\right) \mathrm{j}(\gamma, \tau)^{k} \phi(\gamma(\tau, z))
\end{aligned}
$$

where $\gamma \in S L_{2}(\mathbb{Z})$ and $\lambda, \mu \in \mathbb{Z}$. We say a holomorphic function $\phi: \mathbb{H} \times \mathbb{C} \rightarrow \mathbb{C}$ is an (unrestricted) Jacobi form of weight $k$ and index $m$ for the Jacobi group $S L_{2}(\mathbb{Z}) \ltimes \mathbb{Z}^{2}$ if it is invariant under the above actions, $\phi=\left.\phi\right|_{k, m} \gamma$ and $\phi=\left.\phi\right|_{m}(\lambda, \mu)$, for all $\gamma \in S L_{2}(\mathbb{Z})$ and for all $(\lambda, \mu) \in \mathbb{Z}^{2}$. In what follows, we refer to the transformations (53) and (54) as the elliptic and modular transformations, respectively.

The invariance of $\phi(\tau, z)$ under $\tau \rightarrow \tau+1$ and $z \rightarrow z+1$ implies a Fourier expansion

$$
\phi(\tau, z)=\sum_{n, r \in \mathbb{Z}} c(n, r) q^{n} y^{r}
$$

and the elliptic transformation can be used to show that $c(n, r)$ depends only on the discriminant $D=r^{2}-4 m n$ and on $r(\bmod 2 m)$. In other words, we have $c(n, r)=$ $C_{\widetilde{r}}\left(r^{2}-4 m n\right)$ where $\widetilde{r} \in \mathbb{Z} / 2 m \mathbb{Z}$ and $r=\widetilde{r}(\bmod 2 m)$, for some appropriate function $D \mapsto C_{\widetilde{r}}(D)$. An unrestricted Jacobi form is called a weak Jacobi form, a (strong) Jacobi form, or a Jacobi cusp form when the Fourier coefficients satisfy $c(n, r)=0$ whenever $n<0, C_{\widetilde{r}}(D)=0$ whenever $D>0$, or $C_{\widetilde{r}}(D)=0$ whenever $D \geq 0$, respectively. In a slight 
departure from the notation in [40], we denote the space of weak Jacobi forms of weight $k$ and index $m$ by $J_{k, m}$.

In what follows, we will need two further generalisations of the above definitions. The first is relatively straightforward and replaces $S L_{2}(\mathbb{Z})$ by a finite index subgroup $\Gamma \subset S L_{2}(\mathbb{Z})$ in the modular transformation law. (One has to consider Fourier expansions (55) for each cusp of $\Gamma$ ). The second is more subtle and leads to meromorphic Jacobi forms which obey the modular and elliptic transformation laws but are such that the functions $z \mapsto \phi(\tau, z)$ are allowed to have poles lying at values of $z \in \mathbb{C}$ corresponding to torsion points of the elliptic curve $\mathbb{C} /(\mathbb{Z} \tau+\mathbb{Z})$. Our treatment of meromorphic Jacobi forms ( $c f$. 'From Meromorphic Jacobi forms to mock modular forms' section) mostly follows [38] and [41]. We will only consider functions with simple poles in $z$.

The elliptic transformation (53) implies (cf. [40]) that a (weak) Jacobi form of weight $k$ and index $m$ admits an expansion

$$
\phi(\tau, z)=\sum_{r(\bmod 2 m)} \widetilde{h}_{m, r}(\tau) \theta_{m, r}(\tau, z)
$$

in terms of the index $m$ theta functions,

$$
\theta_{m, r}(\tau, z)=\sum_{\substack{k \in \mathbb{Z} \\ k=r(\bmod 2 m)}} q^{k^{2} / 4 m} y^{k} .
$$

Recall that the vector-valued function $\theta_{m}=\left(\theta_{m, r}\right)$ satisfies

$$
\theta_{m}\left(-\frac{1}{\tau},-\frac{z}{\tau}\right)=\sqrt{-i \tau} e\left(\frac{m z^{2}}{\tau}\right) \mathbf{S} \theta_{m}(\tau, z), \quad \theta_{m}(\tau+1, z)=\mathbf{T} \theta_{m}(\tau, z),
$$

where $\mathbf{S}$ and $\mathbf{T}$ are the $2 m \times 2 m$ unitary matrices with entries

$$
\mathbf{S}_{r r^{\prime}}=\frac{1}{\sqrt{2 m}} e\left(\frac{r r^{\prime}}{2 m}\right), \quad \mathbf{T}_{r r^{\prime}}=e\left(\frac{r^{2}}{4 m}\right) \delta_{r, r^{\prime}}
$$

From this, we can see that $\tilde{h}=\left(\tilde{h}_{m, r}\right)$ is a $2 m$-component vector transforming as a weight $k-1 / 2$ modular form for $S L_{2}(\mathbb{Z})$, with a multiplier system represented by the matrices $\mathbf{S}^{\dagger}$ and $\mathbf{T}^{\dagger}$, satisfying $\mathbf{S S}^{\dagger}=\mathbf{T T}^{\dagger}=I_{2 m}$ and corresponding to the modular transformations $S$ and $T$, respectively. (See [42]). Moreover, the invariance under the modular transformation (54) with $\gamma=-I_{2}$ implies that $\phi(\tau,-z)=(-1)^{k} \phi(\tau, z)$. Combining this with the identity $\theta_{m,-r}(\tau, z)=\theta_{m, r}(\tau,-z)$, we see that

$$
\widetilde{h}_{m, r}=(-1)^{k} \tilde{h}_{m,-r} .
$$

\section{The Eichler-Zagier operators and an ADE classification}

We now turn to a discussion of the Eichler-Zagier operators on Jacobi forms and establish an $\mathrm{ADE}$ classification of maps satisfying a certain positivity condition.

Recall from the 'Jacobi forms' section that a Jacobi form of weight $k$ and index $m$ admits a decomposition (56) into a combination of theta functions $\theta_{m, r}$ and the $2 m$ components $\widetilde{h}_{m, r}$ of a vector-valued modular form $\widetilde{h}_{m}$ of weight $k-1 / 2$. On the other hand, one can also consider the following question: for a given vector-valued modular form $\widetilde{h}_{m}$, is the expression in (56) the only combination of $\theta_{m, r}$ and $\widetilde{h}_{m, r}$ that has the right transformation 
property to be a weight $k$, index $m$ Jacobi form? In other words, we would like to consider all $2 m \times 2 m$ matrices $\Omega$ such that

$$
\tilde{h}_{m}^{T} \cdot \Omega \cdot \theta_{m}=\sum_{r, r^{\prime}(\bmod 2 m)} \widetilde{h}_{m, r} \Omega_{r, r^{\prime}} \theta_{m, r^{\prime}}
$$

is again a weight $k$ index $m$ Jacobi form.

From (58) to (59) as well as the transformation under $\gamma=-I_{2}$, we see that this condition amounts to considering the commutants $\Omega$ of $\mathbf{S}$ and $\mathbf{T}$ satisfying

$$
\mathbf{S}^{\dagger} \Omega \mathbf{S}=\mathbf{T}^{\dagger} \Omega \mathbf{T}=\Omega .
$$

In particular, as $\Omega$ commutes with $\mathbf{S}^{2}$, we see that it has the reflection symmetry

$$
\Omega_{r, r^{\prime}}=\Omega_{-r,-r^{\prime}}
$$

Such commutants have been classified in [43]. For each positive integer $m$, the space of $2 m \times 2 m$ matrices satisfying (62) has dimension given by the number of divisors of $m, \sigma_{0}(m)=\sum_{d \mid m} 1$, and is spanned by the set of linearly independent matrices $\left\{\Omega_{m}\left(n_{1}\right), \Omega_{m}\left(n_{2}\right), \ldots, \Omega_{m}\left(n_{\sigma_{0}(m)}\right)\right\}$ whose entries are given by

$$
\Omega_{m}\left(n_{i}\right)_{r, r^{\prime}}= \begin{cases}1 & \text { if } r+r^{\prime}=0 \bmod 2 n_{i} \text { and } r-r^{\prime}=0 \bmod 2 m / n_{i}, \\ 0 & \text { otherwise, }\end{cases}
$$

where $1=n_{1}<n_{2}<\cdots<m=n_{\sigma_{0}(m)}$ are the divisors of $m$. It is easy to check that these matrices automatically satisfy (63).

Note that

$$
\tilde{h}_{m}^{T} \cdot \Omega_{m}(n) \cdot \theta_{m}=(-1)^{k} \tilde{h}_{m}^{T} \cdot \Omega_{m}(m / n) \cdot \theta_{m},
$$

as is evident from the definition (64) of $\Omega_{m}(n)$ as well as the reflection property (60) of the components $\widetilde{h}_{m, r}$ of $\widetilde{h}_{m}$.

In fact, as we will now show, for a given vector-valued modular form $\widetilde{h}_{m}=\left(\widetilde{h}_{m, r}\right)$ and any given divisor $n$ of $m$, the new Jacobi form $\widetilde{h}_{m}^{T} \cdot \Omega_{m}(n) \cdot \theta_{m}$ can be obtained from the original one $\widetilde{h}_{m}^{T} \cdot \theta_{m}$ via a natural operator - the so-called Eichler-Zagier operator [40] on Jacobi forms.

Given positive integers $n, m$ such that $n \mid m$, we define an Eichler-Zagier operator $\mathcal{W}_{m}(n)$ acting on a function $f: \mathbb{H} \times \mathbb{C} \rightarrow \mathbb{C}$ by setting

$$
\left(f \mid \mathcal{W}_{m}(n)\right)(\tau, z)=\frac{1}{n} \sum_{a, b=0}^{n-1} e\left(m\left(\frac{a^{2}}{n^{2}} \tau+2 \frac{a}{n} z+\frac{a b}{n^{2}}\right)\right) f\left(\tau, z+\frac{a}{n} \tau+\frac{b}{n}\right) .
$$

It is easy to see that the operator $\mathcal{W}_{m}(n)$ commutes with the index $m$ elliptic transformation (53)

$$
f\left|\mathcal{W}_{m}(n)\right|_{m}(\lambda, \mu)=\left.f\right|_{m}(\lambda, \mu) \mid \mathcal{W}_{m}(n)
$$

for all $\mu, \lambda \in \mathbb{Z}$ and in particular preserves the invariance under elliptic transformations. Moreover, one can easily check that the modular invariance (54) is also preserved. As a result, $\mathcal{W}_{m}(n)$ maps an unrestricted Jacobi form of weight $k$ and index $m$ to another unrestricted Jacobi form of the same weight and index. Moreover, when $n \| m$, this operation is an involution on the space of strong Jacobi forms. This involution is sometimes referred to as an Atkin-Lehner involution for Jacobi forms due to its intimate relation to AtkinLehner involutions for modular forms [44,45]. We will explain and utilise some aspects of this relation in the 'From Niemeier lattices to principal moduli' section. 
For later use, we define the more general operator $\mathcal{W}=\sum_{n_{i} \mid m} c_{i} \mathcal{W}_{m}\left(n_{i}\right)$ by setting

$$
f \mid \mathcal{W}=\sum_{n_{i} \mid m} c_{i}\left(f \mid \mathcal{W}_{m}\left(n_{i}\right)\right)
$$

The relation between the Eichler-Zagier operators $\mathcal{W}$ and the transformation on Jacobi forms

$$
\tilde{h}_{m}^{T} \cdot \theta_{m} \mapsto \tilde{h}_{m}^{T} \cdot \Omega \cdot \theta_{m}
$$

where $\Omega$ is a linear combination of the matrices $\Omega_{m}(n)$ in (64), can be seen via the action of the former on the theta functions $\theta_{m, r}$. Notice that

$$
\theta_{m, r} \mid \mathcal{W}_{m}(n)=\sum_{r^{\prime}(\bmod 2 m)} \Omega_{m}(n)_{r, r^{\prime}} \theta_{m, r^{\prime}}
$$

In terms of the $2 m$-component vector $\theta_{m}=\left(\theta_{m, r}\right)$, we have

$$
\theta_{m} \mid \mathcal{W}_{m}(n)=\Omega_{m}(n) \cdot \theta_{m},
$$

which immediately leads to

$$
\tilde{h}_{m}^{T} \cdot \theta_{m} \mid \mathcal{W}_{m}(n)=\widetilde{h}_{m}^{T} \cdot \Omega_{m}(n) \cdot \theta_{m} .
$$

In other words, the Jacobi forms we discussed above in terms of the matrices $\Omega_{m}(n)$ are simply the images of the original Jacobi forms under the corresponding Eichler-Zagier operators. This property makes it obvious that $\tilde{h}_{m}^{T} \cdot \Omega_{m}(n) \cdot \theta_{m}$ is also a Jacobi form since $\mathcal{W}_{m}(n)$ preserves the transformation under the Jacobi group. This relation will be important in the discussion in the 'The umbral mock modular forms' section.

Apart from the modularity (Jacobi form) condition, it is also natural to impose a certain positivity condition. As we will see, this additional condition leads to an ADE classification of the matrices $\Omega$. To explain this positivity condition, first recall that all the entries of the matrices $\Omega_{m}(n)$ for any divisor $n$ of $m$ are non-negative integers and it might seem that any positivity condition would be redundant. However, we have also seen that the description of the theta-coefficients $\widetilde{h}_{m, r}$ of a weight $k$, index $m$ Jacobi form as a vector with $2 m$ components has some redundancy since different components are related to each other by $\tilde{h}_{m, r}=(-1)^{k} \widetilde{h}_{m,-r}(c f$. (60)). For the purpose of the present paper, we will from now on consider only the case of odd $k$, where there are at most $m-1$ independent components in $\left(\tilde{h}_{m, r}\right)$. In this case, using the property (63), we can rewrite the Jacobi form $\tilde{h}_{m}^{T} \cdot \Omega \cdot \theta_{m}$ as

$$
\tilde{h}_{m}^{T} \cdot \Omega \cdot \theta_{m}=\sum_{r, r^{\prime}=1}^{m-1} \widetilde{h}_{m, r}\left(\Omega_{r, r^{\prime}}-\Omega_{r,-r^{\prime}}\right)\left(\theta_{m, r^{\prime}}-\theta_{m,-r^{\prime}}\right) .
$$

As a result, it is natural to consider the $2 m \times 2 m$ matrices $\Omega=\sum_{i=1}^{\sigma_{0}(m)} c_{i} \Omega_{m}\left(n_{i}\right)$ where $n_{1}, n_{2}, \ldots$ are the (distinct) divisors of $m$ that satisfy the corresponding positivity and integrality condition

$$
\Omega_{r, r^{\prime}}-\Omega_{r,-r^{\prime}} \in \mathbb{Z}_{\geq 0} \text { for all } r, r^{\prime}=1, \ldots, m-1,
$$

with a natural normalisation

$$
\Omega_{1,1}-\Omega_{1,-1}=1 \text {. }
$$

Evidently, they are in one-to-one correspondence with the non-negative integer combinations of $\left(\tilde{h}_{m, r}\right)$ and $\left(\theta_{m, r^{\prime}}-\theta_{m,-r^{\prime}}\right)$, with $r, r^{\prime}=1,2, \ldots, m-1$, with the coefficient of 
the term $\tilde{h}_{m, 1} \theta_{m, 1}$ equal to 1 . From a conformal field theory point of view, this is precisely the requirement of having a unique ground state in the theory.

It turns out that this problem has been studied by Cappelli et al. [24]. They found a beautiful ADE classification of such $2 m \times 2 m$ matrices $\Omega$ (see Proposition 2 of [24]), and we present these matrices in Table 6 , denoting by $\Omega^{X}$ the matrix corresponding to the irreducible simply laced root system $X$. The motivation of [24] was very different from ours: these authors were interested in classifying the modular invariant combinations of chiral and anti-chiral characters of the affine Lie algebra $\widehat{A}_{1}$ (the $S U(2)$ current algebra). However, as the modular transformation of the $\widehat{A}_{1}$ characters at level $m-2$ is very closely related to that of the index $m$ theta functions $\theta_{m, r}$, the relevant matrices are also the commutants of the same $\mathbf{S}$ and $\mathbf{T}$ matrices satisfying (62).

The relation between the Eichler-Zagier operators and the $\Omega_{m}(n)$ matrices discussed earlier makes it straightforward to extend the above ADE classification to an ADE classification of Eichler-Zagier operators. Combining the results of the above discussion, we arrive at the following theorem.

Theorem 1. For any integer $m$ and any odd integer $k$, and any vector-valued modular form $\tilde{h}_{m}=\left(\tilde{h}_{m, r}\right)$ such that $\tilde{h}_{m}^{T} \cdot \theta_{m}$ is an (unrestricted) weight $k$, index $m$ Jacobi form, suppose $\Omega$ coincides with a matrix $\Omega^{X}$ corresponding to an irreducible simply laced root system $X$ with Coxeter number $m$ via Table 6 . Then, the combination $\tilde{h}_{m}^{T} \cdot \Omega \cdot \theta_{m}$ is also a weight $k$, index $m$ (unrestricted) Jacobi form which moreover satisfies the positivity condition

$$
\tilde{h}_{m}^{T} \cdot \Omega \cdot \theta_{m}=\sum_{r, r^{\prime}=1}^{m-1} c_{r, r^{\prime}} \tilde{h}_{m, r}\left(\theta_{m, r^{\prime}}-\theta_{m,-r^{\prime}}\right), c_{r, r^{\prime}} \in \mathbb{Z}_{\geq 0}, c_{1,1}=1 .
$$

Conversely, any $2 m \times 2 m$ matrix $\Omega$ for which the above statement is true necessarily coincides with a matrix $\Omega^{X}$ corresponding to an irreducible simply laced root system $X$ with Coxeter number $m$. Moreover, the resulting (unrestricted) Jacobi form is the image of the original Jacobi form $\tilde{h}_{m}^{T} \cdot \theta_{m}$ under the Eichler-Zagier operator $\mathcal{W}^{X}$ defined by replacing $\Omega_{m}(n)$ in $\Omega^{X}$ with $\mathcal{W}_{m}(n)(c f .(78))$.

Proof. First, the Jacobi form condition on $\widetilde{h}_{m}^{T} \cdot \Omega \cdot \theta_{m}$ requires that $\Omega$ satisfies the commutant condition (62). It was shown in [43] (cf. Proposition 1 of [24]) that the space of such $2 m \times 2 m$ matrices are spanned by $\left\{\Omega_{m}\left(n_{1}\right), \Omega_{m}\left(n_{2}\right), \ldots, \Omega_{m}\left(n_{\sigma_{0}(m)}\right)\right\}$ given in (64).

Next, the positivity and integrality conditions on $c_{r, r^{\prime}}$ are equivalent to those on the entries $\Omega_{r, r^{\prime}}-\Omega_{r,-r^{\prime}}$ given in (74-75). The linear combinations of $\Omega_{m}\left(n_{i}\right)$ satisfying (74$75)$ were shown in [24] to correspond to ADE root systems via Table 6 . Finally, the equality (78) follows from the equality (71).

Table 6 The ADE classification of matrices $\Omega$ producing Jacobi forms $\tilde{h}_{m}^{T} \cdot \Omega \cdot \theta_{m}$

\begin{tabular}{lccc}
\hline $\boldsymbol{X}$ & $\boldsymbol{m}(\boldsymbol{X})$ & $\boldsymbol{\pi}^{\boldsymbol{X}}$ & $\boldsymbol{\Omega}^{\boldsymbol{X}}$ \\
\hline$A_{m-1}$ & $m$ & $\frac{m}{1}$ & $\Omega_{m}(1)$ \\
$D_{m / 2+1}$ & $\frac{2 . m}{1 .(m / 2)}$ & $\Omega_{m}(1)+\Omega_{m}(m / 2)$ \\
$E_{6}$ & $m$ & $\frac{2.3 .12}{1.4 .6}$ & $\Omega_{12}(1)+\Omega_{12}(4)+\Omega_{12}(6)$ \\
$E_{7}$ & 12 & $\frac{2.3 .18}{1.6 .9}$ & $\Omega_{18}(1)+\Omega_{18}(6)+\Omega_{18}(9)$ \\
$E_{8}$ & 18 & $\frac{2.3 .5 .30}{1.6 .10 .15}$ & $\Omega_{30}(1)+\Omega_{30}(6)+\Omega_{30}(10)+\Omega_{30}(15)$ \\
\hline
\end{tabular}


The relation between $\Omega^{X}$ and the ADE root system $X$ lies in the following two facts. First, $\Omega^{X}$ is a $2 m \times 2 m$ matrix where $m$ is the Coxeter number of $X$. Moreover, $\Omega_{r, r}^{X}-$ $\Omega_{r,-r}^{X}=\alpha_{r}^{X}$ for $r=1, \ldots, m-1$ coincides with the multiplicity of $r$ as a Coxeter exponent of $X$ ( $c f$. Table 3). Note the striking similarity between the expression for $\Omega^{X}$ and the denominator of the Coxeter Frame shape $\pi^{X}$ (cf. 'Root systems' section). For instance, $\Omega^{X}=\Omega_{m}(1)$ for $X=A_{m-1}$ is nothing but the $2 m \times 2 m$ identity matrix.

More generally, for a union $X=\bigcup_{i} X_{i}$ of simply laced root systems with the same Coxeter number, we let

$$
\Omega^{X}=\sum_{i} \Omega^{X_{i}} \text {, and similarly } \mathcal{W}^{X}=\sum_{i} \mathcal{W}^{X_{i}}
$$

Then, we have the relation

$$
\left(\widetilde{h}_{m}^{T} \cdot \theta_{m}\right) \mid \mathcal{W}^{X}=\widetilde{h}_{m}^{T} \cdot \Omega^{X} \cdot \theta_{m}
$$

among different (odd) weight $k$ and index $m$ Jacobi forms corresponding to the same vector-valued modular form $\widetilde{h}_{m}=\left(\tilde{h}_{m, r}\right)$. Note that the operator $\mathcal{W}^{X}$ is in general no longer an involution and often not even invertible. The Eichler-Zagier operators $\mathcal{W}^{X}$ corresponding to Niemeier root systems ( $c f$. 'Lattices' section) will play a central role in the 'The umbral McKay-Thompson series' section.

The $\widehat{A}_{1}$ characters, which have led to the ADE classification of Cappelli et al., are rather ubiquitous in two-dimensional conformal field theory. They can be viewed as the building blocks of, for instance, the characters of $N=4$ super-conformal algebra and the partition functions of $N=2$ minimal models ( $c f$., e.g. $[9,43]$ ). Moreover, the positivity and the integrality condition (74-75) that are necessary to obtain the ADE classification are completely natural from the point of view of the conformal field theories. This might be seen as suggesting a relationship between umbral moonshine and two-dimensional conformal field theories. The concrete realisation of such a relationship is beyond the scope of the present paper.

\section{From Meromorphic Jacobi forms to mock modular forms}

In the 'Mock modular forms' section, we have seen the definition of mock modular form and its vector-valued generalisation. One of the natural places where such vector-valued mock modular forms occur is in the theta expansion of meromorphic Jacobi forms. To be more precise, following [38] and [41], we will establish a uniform way to separate a meromorphic Jacobi form $\psi$ into its polar and finite parts

$$
\psi(\tau, z)=\psi^{P}(\tau, z)+\psi^{F}(\tau, z) .
$$

The finite part will turn out to be a mock Jacobi form, admitting a theta expansion as in (56), whose theta coefficients are the components of a vector-valued mock modular form. Up to this point, all the mock modular forms playing a role in umbral moonshine, as well as other interesting examples including many of Ramunanjan's mock theta functions, can be obtained in this way as theta coefficients of finite parts of meromorphic Jacobi forms.

For the purpose of this paper, we will focus on the case of weight 1 Jacobi forms with simple poles as a function of $z$. Consider such a Jacobi form $\psi$ with a pole at $z=z_{s}$, where $z_{s}$ is a point inside the fundamental cell $\alpha \tau+\beta, \alpha, \beta \in(-1,0]$. The elliptic transformation (53) then forces $\psi$ to have poles at all $z \in z_{s}+\mathbb{Z}+\tau \mathbb{Z}$. With this property in mind, 
in the rest of the paper, we will only write down the location of the poles inside the fundamental cell. To capture this translation property of the poles, following [41], we define an averaging operator

$$
\operatorname{Av}_{m}[F(y)]=\sum_{k \in \mathbb{Z}} q^{m k^{2}} y^{2 m k} F\left(q^{k} y\right)
$$

which takes a function of $y=e(z)$ with polynomial growth and returns a function of $z$ which is invariant under the index $m$ elliptic transformations (53). For a given pole $z=z_{s}$ of a weight 1 index $m$ meromorphic Jacobi form $\psi$, we will consider the image $\psi_{z_{s}}^{P}$ under $\operatorname{Av}_{m}$ of a suitably chosen meromorphic function $F_{z_{s}}(y)$ that has a pole at $z=z_{s}$, such that $\psi-\psi_{z_{s}}^{P}$ is regular at all $z \in z_{s}+\mathbb{Z}+\tau \mathbb{Z}$.

In the remaining part of this subsection, we will first review (following [38,41]) this construction of mock modular forms in more detail and then extend the discussion of the Eichler-Zagier operators to meromorphic Jacobi forms and study how they act on the polar and the finite part separately. This will allow us to establish an ADE classification of mock Jacobi forms of a specific type in the next section and constitutes a crucial element in the construction of the umbral mock modular forms $H^{X}$.

\section{A simple pole at $z=0$}

To start with, consider a meromorphic Jacobi form $\psi(\tau, z)$ of weight 1 and index $m$, with a simple pole at $z=0$ and no other poles. Define the polar part of $\psi$ to be

$$
\psi^{P}(\tau, z)=\chi(\tau) \operatorname{Av}_{m}\left[\frac{y+1}{y-1}\right]
$$

where $\chi(\tau) / \pi i$ is the residue of $\psi(\tau, z)$ at $z=0$. For the applications in the present paper, we need only consider the case that $\chi(\tau)=\chi$ is a constant. With this definition, one can easily check that $\psi^{F}=\psi-\psi^{P}$ is indeed a holomorphic function with no poles in $z$.

Note that

$$
\mu_{m, 0}(\tau, z)=\operatorname{Av}_{m}\left[\frac{y+1}{y-1}\right],
$$

where we define the generalised Appell-Lerch sum

$$
\mu_{m, j}(\tau, z)=(-1)^{1+2 j} \sum_{k \in \mathbb{Z}} q^{m k^{2}} y^{2 m k} \frac{\left(y q^{k}\right)^{-2 j}+\left(y q^{k}\right)^{-2 j+1}+\cdots+\left(y q^{k}\right)^{1+2 j}}{1-y q^{k}}
$$

for $j \in \frac{1}{2} \mathbb{Z}$. The function $\mu_{m, 0}$ enjoys the following relation to the modular group $S L_{2}(\mathbb{Z})$. Define the completion of $\mu_{m, 0}(\tau, z)$ by setting

$$
\begin{aligned}
& \widehat{\mu}_{m}(\tau, \bar{\tau}, z)=\mu_{m, 0}(\tau, z) \\
& -e\left(-\frac{1}{8}\right) \frac{1}{\sqrt{2 m}} \sum_{r(\bmod 2 m)} \theta_{m, r}(\tau, z) \int_{-\bar{\tau}}^{i \infty}\left(\tau^{\prime}+\tau\right)^{-1 / 2} \overline{S_{m, r}\left(-\bar{\tau}^{\prime}\right)} \mathrm{d} \tau^{\prime},
\end{aligned}
$$

where $S_{m, r}(\tau)$ denotes the unary theta series

$$
S_{m, r}(\tau)=-S_{m,-r}(\tau)=\left.\frac{1}{2 \pi i} \frac{\partial}{\partial z} \theta_{m, r}(\tau, z)\right|_{z=0},
$$

then $\widehat{\mu}_{m}$ transforms like a Jacobi form of weight 1 and index $m$ for $S L_{2}(\mathbb{Z})$ but is clearly no longer holomorphic whenever $m>1$. From the above definition and the transformation (59) of the theta functions, we see that $S_{m}=\left(S_{m, r}\right)$ is a (vector-valued) weight $3 / 2$ cusp form for $S L_{2}(\mathbb{Z})$. 
Returning to our weight 1 index $m$ Jacobi form $\psi$, assumed to have a simple pole at $z=0$, it is now straightforward to see that the finite part $\psi^{F}=\psi-\psi^{P}$, a holomorphic function on $\mathbb{H} \times \mathbb{C}$, has a completion given by

$$
\widehat{\psi}^{F}=\psi^{F}+\chi e\left(-\frac{1}{8}\right) \frac{1}{\sqrt{2 m}} \sum_{r(\bmod 2 m)} \theta_{m, r}(\tau, z) \int_{-\bar{\tau}}^{i \infty}\left(\tau^{\prime}+\tau\right)^{-1 / 2} \overline{S_{m, r}\left(-\bar{\tau}^{\prime}\right)} \mathrm{d} \tau^{\prime}
$$

that transforms like a Jacobi form of weight 1 and index $m$ for $S L_{2}(\mathbb{Z})$. As such, $\psi^{F}$ is an example of a mock Jacobi form (cf. [41], Section 7.2). Since both $\psi$ and $\psi^{P}$ are invariant under the index $m$ elliptic transformation, so is the finite part $\psi^{F}$. This fact guarantees a theta expansion of $\psi^{F}$ analogous to that of a (weak) Jacobi form (56)

$$
\psi^{F}(\tau, z)=\sum_{r(\bmod 2 \mathrm{~m})} h_{r}(\tau) \theta_{m, r}(\tau, z)
$$

where $h=\left(h_{r}\right)$ is a weight $1 / 2$ vector-valued holomorphic function on $\mathbb{H}$ whose completion

$$
\widehat{h}_{r}(\tau)=h_{r}(\tau)+\chi e\left(-\frac{1}{8}\right) \frac{1}{\sqrt{2 m}} \int_{-\bar{\tau}}^{i \infty}\left(\tau^{\prime}+\tau\right)^{-1 / 2} \overline{S_{m, r}\left(-\bar{\tau}^{\prime}\right)} \mathrm{d} \tau^{\prime}
$$

transforms as a weight $1 / 2$ vector-valued modular form with $2 m$ components. As such, we conclude that $h=\left(h_{r}\right)$ is a vector-valued mock modular form for the modular group $S L_{2}(\mathbb{Z})$ with shadow $\chi S_{m}=\left(\chi S_{m, r}\right)$.

Note that $S_{1}$ vanishes identically. This is a reflection of the fact that $\mu_{1,0}$, in contrast to the $\mu_{m, 0}$ for $m>1$, coincides with its completion and is thus (already) a meromorphic Jacobi form of weight 1 and index 1 . By construction, it has simple poles at $z \in \mathbb{Z} \tau+\mathbb{Z}$ and nowhere else, and we also have the explicit formula

$$
\mu_{1,0}(\tau, z)=-i \frac{\theta_{1}(\tau, 2 z) \eta(\tau)^{3}}{\theta_{1}(\tau, z)^{2}}=\frac{y+1}{y-1}-\left(y^{2}-y^{-2}\right) q+\cdots .
$$

(See 'Jacobi theta functions' in Appendix 1 for $\theta_{1}(\tau, z)$ ). The function $\mu_{1,0}$ is further distinguished by being a meromorphic Jacobi form with vanishing finite part, a 'Cheshire cat' in the language of ([41], Section 8.5). It will play a distinguished role in the 'The umbral mock modular forms' section, where it will serve as a device for producing meromorphic Jacobi forms of weight 1 from (weak, holomorphic) Jacobi forms of weight 0 .

\section{Simple poles at $n$-torsion points}

Next, we would like to consider the more general situation in which we have a weight 1 index $m$ meromorphic Jacobi form $\psi$ with simple poles at more general torsion points $z \in \mathbb{Q} \tau+\mathbb{Q}$. We introduce the row vector with two elements $s=(\alpha \beta)$ to label the pole at $z_{s}=\alpha \tau+\beta$ and write $y_{s}=e\left(z_{s}\right)$. For the purpose of this paper, we will restrict our attention to the $n$-torsion points satisfying $n z \in \mathbb{Z} \tau+\mathbb{Z}$, where $n$ is a divisor of the index $m$. Focus on a pole located at say $z_{s}=\alpha \tau+\beta$ with $\alpha, \beta \in \frac{1}{n} \mathbb{Z}$. Again following [41], we require the corresponding polar term to be given by the formula

$$
\psi_{z_{s}}^{P}(\tau, z)=\pi i \operatorname{Res}_{z=z_{s}}(\psi(\tau, z)) \operatorname{Av}_{m}\left[\left(\frac{y}{y_{s}}\right)^{-2 m \alpha} \frac{y / y_{s}+1}{y / y_{s}-1}\right],
$$

generalising (81). One can easily check that $\psi-\psi_{z_{s}}^{P}$ has no pole at $z \in z_{s}+\mathbb{Z} \tau+\mathbb{Z}$. 
As before, the above polar part is invariant under the elliptic transformation by construction. To discuss its variance under the modular group, first notice that the transformation $(\tau, z) \mapsto \gamma(\tau, z)$ maps the pole at $z_{s}$ to a different pole according to $s \mapsto s \gamma$. As a result, to obtain a mock Jacobi form for $S L_{2}(\mathbb{Z})$ from a meromorphic Jacobi form with poles at $n$-torsion points $z_{s}$ (where $n$ is the smallest integer such that $z_{s} \in \frac{\tau}{n} \mathbb{Z}+\frac{1}{n} \mathbb{Z}$ ), we should consider meromorphic Jacobi forms that have poles at all the $n$-torsion points. Moreover, the modular transformation of $\psi$ dictates that the residues of the poles satisfy $D_{s}(\gamma \tau)=D_{s \gamma}(\tau)$, where we have defined, after [41],

$$
D_{s}(\tau)=e\left(m \alpha z_{s}\right) \operatorname{Res}_{z=z_{s}}(\psi(\tau, z)) .
$$

More specifically, we would like to consider the situation where $\psi$ satisfies

$$
\operatorname{Res}_{z=-\frac{a}{n} \tau-\frac{b}{n}} \psi(\tau, z)=\chi e\left(-m a(a \tau+b) / n^{2}\right) / n \pi i \text {, for } a, b=0,1, \ldots, n-1,
$$

corresponding to the simplest case where the function $D_{s}(\tau)$ is just a constant. Without loss of generality, we will also assume for the moment that $\psi$ has no other poles, as the more general situation can be obtained by taking linear combinations. In this case, using (90), it is not hard to see that the polar parts contributed by the poles at these $n$-torsion points are given by the images under the Eichler-Zagier operator $\mathcal{W}_{m}(n)(c f .(66))$ of the polar term contributed by the simple pole at the origin, so that

$$
\psi^{P}=\sum_{a, b=0}^{n-1} \psi_{-\frac{a}{n} \tau-\frac{b}{n}}^{P}=\chi \mu_{m, 0} \mid \mathcal{W}_{m}(n) .
$$

From (84) and the fact that the Eichler-Zagier operators preserve the Jacobi transformations, we immediately see how considering Jacobi forms with simple poles at torsion points leads us to vector-valued mock modular forms with more general shadows. In this case, from (71), (84) and (93), it is straightforward to see that the completion of the polar part

$$
\begin{aligned}
\widehat{\psi}^{P}= & \psi^{P} \\
& -\chi e\left(-\frac{1}{8}\right) \frac{1}{\sqrt{2 m}} \sum_{r, r^{\prime}(\bmod 2 m)} \theta_{m, r}(\tau, z) \Omega_{m}(n)_{r, r^{\prime}} \int_{-\bar{\tau}}^{i \infty}\left(\tau^{\prime}+\tau\right)^{-1 / 2} \overline{S_{m, r^{\prime}}\left(-\bar{\tau}^{\prime}\right)} \mathrm{d} \tau^{\prime}
\end{aligned}
$$

again transforms like a Jacobi form of weight 1 and index $m$ for $S L_{2}(\mathbb{Z})$.

Following the same argument as before, we conclude that the theta coefficients of the finite part

$$
\psi^{F}=\psi-\psi^{P}=\sum_{r(\bmod 2 \mathrm{~m})} h_{r}(\tau) \theta_{m, r}(\tau, z),
$$

define a vector-valued mock modular form $h=\left(h_{r}\right)$, whose completion is given by

$$
\begin{aligned}
& \widehat{h}_{r}(\tau)=h_{r}(\tau) \\
& +\chi e\left(-\frac{1}{8}\right) \frac{1}{\sqrt{2 m}} \sum_{r^{\prime}(\bmod 2 \mathrm{~m})} \Omega_{m}(n)_{r, r^{\prime}} \int_{-\bar{\tau}}^{i \infty}\left(\tau^{\prime}+\tau\right)^{-1 / 2} \overline{S_{m, r^{\prime}}\left(-\bar{\tau}^{\prime}\right)} \mathrm{d} \tau^{\prime},
\end{aligned}
$$

and whose shadow is hence given by a vector of unary theta series whose $r$-th component equals

$$
\sum_{r^{\prime}(\bmod 2 \mathrm{~m})} \Omega_{m}(n)_{r, r^{\prime}} S_{m, r^{\prime}}
$$


where $r \in \mathbb{Z} / 2 m \mathbb{Z}$. In particular, this means that the vector-valued mock modular forms arising from meromorphic Jacobi forms in this way are closely related to mock theta functions, as their shadows are always given by unary theta series.

Finally, we also note that the Eichler-Zagier operators and the operations of extracting polar and finite parts are commutative in the following sense.

Proposition 2. Suppose $\psi$ is a weight 1 index $m$ meromorphic Jacobi form with simple poles at $\tilde{n}$-torsion points with $\widetilde{n} \mid m$ and with no poles elsewhere. Then, for any positive integer $n$ such that $n \mid m$ and $(n, \widetilde{n})=1$, we have

$$
\left(\psi \mid \mathcal{W}_{m}(n)\right)^{P}=\psi^{P} \mid \mathcal{W}_{m}(n) .
$$

Proof. Denote the set of poles of $\psi$ in the unit cell by $S$, and focus on the pole of $\psi$ at $z_{*}=-\tilde{a} / \tilde{n} \tau-\tilde{b} / \tilde{n} \in S$. From the action of $\mathcal{W}_{m}(n)$, we see that $\psi \mid \mathcal{W}_{m}(n)$ has poles at all $z \in z_{*}+\frac{1}{n} \mathbb{Z}+\frac{\tau}{n} \mathbb{Z}$. Focussing on the pole at $z=z_{s}=z_{*}-(a \tau / n+b / n)$, from (66), we get

$$
\operatorname{Res}_{z=z_{s}}\left(\psi \mid \mathcal{W}_{m}(n)\right)(\tau, z)=\frac{1}{n} e\left(\frac{2 m a z_{s}}{n}\right) e\left(\frac{m a}{n^{2}}(a \tau+b)\right) \operatorname{Res}_{z=z_{*}}(\psi(\tau, z)),
$$

which leads to

$$
\begin{aligned}
& \left(\psi \mid \mathcal{W}_{m}(n)\right)^{P}(\tau, z) \\
& =\frac{\pi i}{n} \sum_{z_{*}=-\tilde{\tilde{a}} \tau-\frac{\tilde{\sigma}}{n} \in S} \operatorname{Res}_{z=z_{*}}(\psi(\tau, z)) \\
& \quad \times \sum_{a, b=0}^{n-1} e\left(\frac{2 m a z_{s}}{n}\right) e\left(\frac{m a}{n^{2}}(a \tau+b)\right) \sum_{k \in \mathbb{Z}} q^{m k^{2}} y^{2 m k}\left(q^{k} y / y_{s}\right)^{2 m\left(\frac{a}{n}+\frac{\tilde{a}}{n}\right)} \frac{q^{k} y / y_{s}+1}{q^{k} y / y_{s}-1}
\end{aligned}
$$

where $y_{s}$ denotes $y_{s}=e\left(z_{s}\right)=e(-(\tilde{a} / \tilde{n}+a / n) \tau-(\tilde{b} / \tilde{n}+b / n))$ in the second line.

By direct comparison using (66) and (90), this is exactly $\psi^{P} \mid \mathcal{W}_{m}(n)$ and this finishes the proof.

Since all the operations involved are linear, we also have the following corollary.

Corollary 1. Consider $\psi$ as defined as in Proposition 2 and let $\mathcal{W}=\sum_{i} c_{i} \mathcal{W}_{m}\left(n_{i}\right)$ where the $n_{i}$ are divisors of $m$ satisfying $\left(n_{i}, \widetilde{n}\right)=1$. Then,

$$
(\psi \mid \mathcal{W})^{P}=\psi^{P} \mid \mathcal{W}
$$

and

$$
(\psi \mid \mathcal{W})^{F}=\psi^{F} \mid \mathcal{W} .
$$

Moreover, if we denote the theta coefficients of $\psi^{F}$ by $h_{\psi}=\left(\left(h_{\psi}\right)_{r}\right)$ and its shadow by $S_{\psi}=\left(\left(S_{\psi}\right)_{r}\right)$ with $r \in \mathbb{Z} / 2 m \mathbb{Z}$, then the theta coefficients of $(\psi \mid \mathcal{W})^{F}$ form a vector-valued mock modular form $h_{\psi \mid \mathcal{W} \text { satisfying }}$

$$
h_{\psi \mid \mathcal{W}}=\Omega h_{\psi},
$$

with shadow given by

$$
S_{\psi \mid \mathcal{W}}=\Omega S_{\psi}
$$

where $\Omega=\sum_{i} c_{i} \Omega_{m}\left(n_{i}\right)(c f .(64))$. 
As a result, the relations (78) between different Jacobi forms of the same index can be applied separately to the polar and the finite part. In the present paper, we will mostly be concerned with the application of the above to the case that $\widetilde{n}=1$. For later use, it will be useful to note the following property.

Lemma 1. For $\psi$ and $n$ as defined as in Proposition 2, we have

$$
\psi^{F}\left|\mathcal{W}(n)=-\psi^{F}\right| \mathcal{W}(m / n), \quad \widehat{\psi}^{F}\left|\mathcal{W}(n)=-\widehat{\psi}^{F}\right| \mathcal{W}(m / n)
$$

Proof. From the property (93) of $\psi^{P}$ and the elliptic transformation of $\mu_{m, 0}$, it follows that $\psi^{P}(\tau, z)=-\psi^{P}(\tau,-z)$ and therefore $\psi^{F}(\tau, z)=-\psi^{F}(\tau,-z)$. As such, the vectorvalued mock modular form $h=\left(h_{r}\right)$ arising from the theta expansion (87) of $\psi^{F}$ satisfies $h_{r}=-h_{-r}$. Together with $S_{m, r}=-S_{m,-r}$, the lemma follows from the action of $\mathcal{W}_{m}(n)$ (71) on $\theta_{m}=\left(\theta_{m, r}\right)$ and the definition (64) of the matrix $\Omega_{m}(n)$.

\section{The umbral mock modular forms}

Following the general discussion of the relevant automorphic objects in the previous section, in this section, we will start specifying concretely the vector-valued mock modular forms which encode, according to our conjecture, the graded dimensions of certain infinite-dimensional modules for the umbral groups defined in the 'Groups' section. We will specify the shadows of these functions - the umbral mock modular forms - in the 'The umbral shadows' section. Subsequently, in the 'From Niemeier lattices to principal moduli' section, we will show how these shadows distinguish the Niemeier root systems through a relation to genus zero groups and their principal moduli. Afterwards, we will provide explicit expressions for the umbral forms of the A-type Niemeier root systems by specifying a set of weight 0 weak Jacobi forms. The umbral forms of D- and E-type Niemeier root systems will be specified in the next section.

\section{The umbral shadows}

In the 'From Meromorphic Jacobi forms to mock modular forms' section, we have seen how the theta expansion of the finite part of a meromorphic Jacobi form gives rise to a vector-valued mock modular form, and how different configurations of poles lead to different shadows. The shadows of the mock modular forms obtained in this way are always given by unary theta series. In this subsection, we will see how the ADE classification discussed in the 'The Eichler-Zagier operators and an ADE classification' section leads to particular cases of the above construction. Moreover, by combining the ADE classification and the construction of mock modular forms from meromorphic Jacobi forms discussed in the 'From Meromorphic Jacobi forms to mock modular forms' section, we will associate a specific shadow $S^{X}$, or equivalently a pole structure of the corresponding meromorphic Jacobi form $\psi^{X}$, to each of the Niemeier root systems $X$.

Consider a meromorphic Jacobi form $\psi$ with weight 1 and index $m$. Recall from (93) that the contribution to its polar part $\psi^{P}$ from the simple poles at the $n$-torsion points with residues satisfying (92) is given by

$$
\sum_{a, b=0}^{n-1} \psi_{-\frac{a}{n} \tau-\frac{b}{n}}^{P}=\chi \mu_{m, 0} \mid \mathcal{W}_{m}(n)
$$


Clearly, one may consider a linear combination of expressions as in (106). Consider

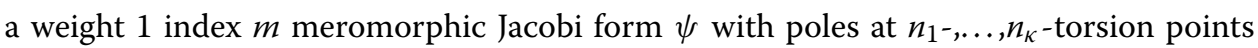
where $n_{j} \mid m$, and where each $n_{j}$ contributes $c_{j} e\left(-m a(a \tau+b) / n_{j}^{2}\right) / n_{j} \pi i$ to the residue of the pole located at $-\frac{a}{n_{j}} \tau-\frac{b}{n_{j}}$. From the above discussion, it follows that its polar part is given by

$$
\psi^{P}=\mu_{m, 0} \mid \mathcal{W}, \text { where } \mathcal{W}=\sum_{i=1}^{\kappa} c_{i} \mathcal{W}_{m}\left(n_{i}\right) .
$$

By taking a linear combination of expressions as in (94), we see that its completion, given by

$$
\begin{aligned}
\widehat{\psi}^{P}= & \psi^{P} \\
& -e\left(-\frac{1}{8}\right) \frac{1}{\sqrt{2 m}} \sum_{r, r^{\prime}(\bmod 2 m)} \theta_{m, r}(\tau, z) \Omega_{r, r^{\prime}} \int_{-\bar{\tau}}^{i \infty}\left(\tau^{\prime}+\tau\right)^{-1 / 2} \overline{S_{m, r^{\prime}}\left(-\bar{\tau}^{\prime}\right)} \mathrm{d} \tau^{\prime},
\end{aligned}
$$

where $\Omega=\sum_{i=1}^{\kappa} c_{i} \Omega_{m}\left(n_{i}\right)$, transforms like a Jacobi form of weight 1 and index $m$ for $S L_{2}(\mathbb{Z})$. Immediately, we conclude that the theta coefficients of the finite part $\psi^{F}=$ $\psi-\psi^{P}$ constitute a vector-valued mock modular form with (the $r$-th component of) the shadow given by

$$
\sum_{r^{\prime}(\bmod 2 m)} \Omega_{r, r^{\prime}} S_{m, r^{\prime}}
$$

Now, recall that in the 'The Eichler-Zagier operators and an ADE classification' section, we used the reflection property $\widetilde{h}_{m, r}=-\widetilde{h}_{m,-r}$ to impose a positivity condition which then led to an ADE classification ( $c f$. Theorem 1). Analogously, in the context of meromorphic Jacobi forms, we also have a natural positivity condition that we want to impose. Using the reflection property $S_{m, r}=-S_{m,-r}$, for $r \in \mathbb{Z} / 2 m \mathbb{Z}$ (cf. (85)), we may instead consider a $(m-1)$-component vector with the $r$-th component given by

$$
\sum_{r^{\prime}=1}^{m-1}\left(\Omega_{r, r^{\prime}}-\Omega_{r,-r^{\prime}}\right) S_{m, r^{\prime}}, \quad r=1, \ldots, m-1 .
$$

Requiring that each component of this $(m-1)$-component vector is a non-negative linear combination of the unary theta series $S_{m, r}$, for $r=1,2, \ldots, m-1$, with the normalisation $\Omega_{1,1}-\Omega_{1,-1}=1$, from Theorem 1 we immediately see that such $\Omega$ (and, equivalently, $\mathcal{W}$ ) are classified by ADE root systems. More precisely, to each irreducible simply laced root system $X$ with Coxeter number $m$, we associate a $2 m$-vector-valued cusp form $S^{X}$, of weight $3 / 2$ for $S L_{2}(\mathbb{Z})$, with $r$-th component given by

$$
S_{r}^{X}=\sum_{r^{\prime}(\bmod 2 m)} \Omega_{r, r^{\prime}}^{X} S_{m, r^{\prime}},
$$

where $m$ denotes the Coxeter number of $X$ and the matrix $\Omega^{X}$ is defined as in Table 6. For instance, we have

$$
S_{r}^{A_{m-1}}=S_{m, r} .
$$

For the D-series root systems with even rank, we have

$$
S_{r}^{D_{2 n}}= \begin{cases}S_{4 n-2, r}+S_{4 n-2,4 n-2-r} & \text { if } r \text { is odd, } \\ 0 & \text { if } r \text { is even }\end{cases}
$$


and in the case that $n$ is odd, we have

$$
S_{r}^{D_{n}}= \begin{cases}S_{2 n-2, r} & \text { if } r \text { is odd, } \\ S_{2 n-2,2 n-2-r} & \text { if } r \text { is even. }\end{cases}
$$

For $E_{6}, E_{7}$ and $E_{8}$ we have

$$
\begin{aligned}
& S_{r}^{E_{6}}= \begin{cases}S_{12,1}+S_{12,7} & \text { if } r=1 \text { or } r=7, \\
S_{12,4}+S_{12,8} & \text { if } r=4 \text { or } r=8, \\
S_{12,5}+S_{12,11} & \text { if } r=5 \text { or } r=11, \\
0 & \text { otherwise; }\end{cases} \\
& S_{r}^{E_{7}}= \begin{cases}S_{18,1}+S_{18,17} & \text { if } r=1 \text { or } r=17, \\
S_{18,9} & \text { if } r=3 \text { or } r=15, \\
S_{18,5}+S_{18,13} & \text { if } r=5 \text { or } r=13, \\
S_{18,7}+S_{18,11} & \text { if } r=7 \text { or } r=11, \\
S_{18,3}+S_{18,9}+S_{18,15} & \text { if } r=9, \\
0 & \text { otherwise; }\end{cases} \\
& S_{r}^{E_{8}}= \begin{cases}S_{30,1}+S_{30,11}+S_{30,19}+S_{30,29} & \text { if } r \in\{1,11,19,29\}, \\
S_{30,7}+S_{30,13}+S_{30,17}+S_{30,23} & \text { if } r \in\{7,13,17,23\}, \\
0 & \text { otherwise; }\end{cases}
\end{aligned}
$$

where for simplicity, we have only specified $S_{r}^{E_{n}}$ for $r \in\{1, \cdots, m-1\} \subset \mathbb{Z} / 2 m \mathbb{Z}$. The remaining components are determined by the rule $S_{-r}^{E_{n}}=-S_{r}^{E_{n}}$.

Following (77), more generally for a union $X=\bigcup_{i} X_{i}$ of simply laced root systems with the same Coxeter number, we have $S^{X}=\sum_{i} S^{X_{i}}$. With this definition, $S^{X}$ is given by the matrix $\Omega^{X}$ as

$$
S^{X}=\left(S_{r}^{X}\right), \quad S_{r}^{X}=\sum_{r^{\prime}(\bmod 2 m)} \Omega_{r, r^{\prime}}^{X} S_{m, r^{\prime}} .
$$

From the above discussion, we see that the cusp form $S^{X}$ arises naturally as the shadow of a vector-valued mock modular form obtained from the theta expansion of a meromorphic Jacobi form $\psi^{X}$ with simple poles at $z \in \frac{\tau}{n_{i}} \mathbb{Z}+\frac{1}{n_{i}} \mathbb{Z}$ for all $n_{i} \mid m$ such that $c_{i}>0$ in $\Omega^{X}=\sum_{i} c_{i} \Omega_{m}\left(n_{i}\right)$, whose polar part is given by

$$
\left(\psi^{X}\right)^{P}=\mu_{m, 0}(\tau, z) \mid \mathcal{W}^{X} .
$$

It is not hard to see that such meromorphic Jacobi forms exist for any simply laced root system $X$ where all the irreducible components have the same Coxeter number. Choose an arbitrary weight 0 , index $m-1$ weak Jacobi form $\phi$ with $\phi(\tau, 0) \neq 0$ and assume, without loss of generality, that $\phi(\tau, 0)=1$. Recall from the definition of weak Jacobi forms in the 'Jacobi forms' section that if $\phi(\tau, z)$ is a weight 0 weak Jacobi form, then $\phi(\tau, 0)$ is a weight 0 modular form, which is necessarily a constant. As such, by multiplying with the meromorphic Jacobi form $\mu_{1,0}$ of weight 1 and index 1 (cf. (89)), we obtain a weight 1 index $m$ meromorphic Jacobi form with simple poles at $z \in \mathbb{Z} \tau+\mathbb{Z}$ and nowhere else, and with the polar part given by $\mu_{m, 0}$. (See [1], Section 2.3 , for a more detailed discussion, but note that $\mu_{1,0}$ is denoted there also by $\left.\Psi_{1,1}\right)$. The Corollary 1 then shows that its 
image $-\mu_{1,0} \phi \mid \mathcal{W}^{X}$ under the corresponding Eichler-Zagier operator is a weight 1 index $m$ meromorphic Jacobi with polar part coinciding with (119).

Besides specifying the poles of the meromorphic Jacobi form, in what follows, we will also require an optimal growth condition (equivalent to the optimal growth condition formulated in [41], with its name derived from the fact that it guarantees the slowest possible growth of the coefficients of the corresponding mock Jacobi form). It turns out that there does not always exist a meromorphic Jacobi form with polar part given by (119) that moreover satisfies this optimal growth condition for an arbitrary simply laced root system $X$, but when it does exist, it is unique.

Theorem 2. Let X be a simply laced root system with all irreducible components having the same Coxeter number $m$. There exists at most one weight 1 index $m$ meromorphic Jacobi form $\psi^{X}$ satisfying the following two conditions. First, its polar part $\left(\psi^{X}\right)^{P}$ is given by (119). Second, its finite part

$$
\left(\psi^{X}\right)^{F}=\psi^{X}-\left(\psi^{X}\right)^{P}=\sum_{r(\bmod 2 m)} h_{r}^{X} \theta_{m, r}
$$

satisfies the optimal growth condition

$$
q^{1 / 4 m} h_{r}^{X}(\tau)=O(1)
$$

as $\tau \rightarrow i \infty$ for all $r \in \mathbb{Z} / 2 m \mathbb{Z}$.

Proof. If there are two distinct meromorphic Jacobi forms satisfying the above conditions, then their difference is necessarily a weight 1 index $m$ weak Jacobi form of optimal growth in the sense of [41]. But Theorem 9.7 of [41] is exactly the statement that no such weak Jacobi form exists.

Corollary 2. Let $X$ be a simply laced root system with all irreducible components having the same Coxeter number $m$. Then, there exists at most one vector-valued mock modular form $h^{X}$ for $S L_{2}(\mathbb{Z})$ with shadow $S^{X}$ that satisfies the optimal growth condition (121).

Proof. Let $h^{X}$ be a vector-valued mock modular form satisfying the above conditions. Consider $\psi^{\prime}=\left(\psi^{X}\right)^{P}+\sum_{r} h_{r}^{X} \theta_{m, r}$ with $\left(\psi^{X}\right)^{P}$ given by (119). From the fact that the multiplier of a mock modular form is the inverse of that of its shadow and from the discussion in the 'From Meromorphic Jacobi forms to mock modular forms' section, $\psi$ ' is a weight 1 index $m$ meromorphic Jacobi form satisfying the conditions of Theorem 2 . It then follows from Theorem 2 that such $h^{X}$ is unique if it exists.

So far, our discussion has been very general, applicable to any simply laced root system with all irreducible components having the same Coxeter number. In the next subsection, we will see how the cusp forms $S^{X}$ with $X$ given by one of the 23 Niemeier root systems play a distinguished role. The $S^{X}$ for $X$ a Niemeier root system are called the umbral shadows,

$$
S_{r}^{X}=\sum_{r^{\prime} \in \mathbb{Z} / 2 m \mathbb{Z}} \Omega_{r, r^{\prime}}^{X} S_{m, r^{\prime}}
$$

We will demonstrate the existence of meromorphic Jacobi forms $\psi^{X}$ satisfying the conditions of Theorem 2 for $X$ an A-type Niemeier root system in Proposition 5 and for D- 
and E-type Niemeier root systems in Proposition 6. The resulting vector-valued functions defined by the theta coefficients of the finite parts of these $\psi^{X}$ are the umbral mock modular forms, to be denoted $H^{X}=\left(H_{r}^{X}\right)$. The first few dozen Fourier coefficients of the umbral mock modular forms are tabulated in Additional file 1. For some of the Niemeier root systems $X$, the meromorphic Jacobi forms $\psi^{X}$ are closely related to some of the meromorphic Jacobi forms analysed in [41], Section 9.5; for other Niemeier root systems $X$, the corresponding shadows $S^{X}$ fall outside the range of analysis in [41], and this is why we find mock Jacobi forms of optimal growth for values of $m$ other than those appearing in the work of Dabholkar, Murthy and Zagier.

\section{From Niemeier lattices to principal moduli}

In the last subsection, we have seen how ADE root systems have an intimate relation to meromorphic Jacobi forms. More precisely, to a simply laced root system with all irreducible components having the same Coxeter number $m$, we associate a pole structure for meromorphic Jacobi forms of weight 1 and index $m$. Equivalently, we associate a weight $3 / 2$ vector-valued cusp form to every such root system, which plays the role of the shadow of the mock modular form arising from the meromorphic Jacobi form via the relation discussed in the 'From Meromorphic Jacobi forms to mock modular forms' section. In this subsection, we see how the shadows $S^{X}$ attached to Niemeier root systems are distinguished and, in particular, how they are related to the genus zero groups $\Gamma^{X}$ of the 'Genus zero groups' section.

Recall [45] that a skew-holomorphic Jacobi form of weight 2 and index $m$ is a smooth function $\widetilde{\phi}(\tau, z)$ on $\mathbb{H} \times \mathbb{C}$ which is periodic in both $\tau$ and $z$ with period 1, transforms under the $S$-transformation as

$$
\widetilde{\phi}\left(-\frac{1}{\tau}, \frac{z}{\tau}\right) e\left(-m \frac{z^{2}}{\tau}\right)=\bar{\tau}|\tau| \widetilde{\phi}(\tau, z)
$$

and has a Fourier expansion

$$
\tilde{\phi}(\tau, z)=\sum_{\substack{\Delta, r \in \mathbb{Z} \\ \Delta=r^{2}(\bmod 4 m)}} C_{\widetilde{\phi}}(\Delta, r) \mathrm{e}\left(\frac{r^{2}-\Delta}{4 m} \Re(\tau)+\frac{r^{2}+|\Delta|}{4 m} i \Im(\tau)+r z\right)
$$

where $C_{\widetilde{\phi}}(\Delta, r)=0$ for $\Delta<0$. We denote the space of such functions by $J_{2, m}^{+}$. Recall that an integer $\Delta$ is called a fundamental discriminant if $\Delta=1$ or $\Delta$ is the discriminant of a quadratic number field. Following Skoruppa [45] (see also [46]), given a pair $\left(\Delta_{0}, r_{0}\right)$ where $\Delta_{0}$ is a positive fundamental discriminant that is a square modulo $4 m$ and $\Delta_{0}=r_{0}^{2}$ $(\bmod 4 m)$, we may associate a weight 2 modular form

$$
\mathscr{S}_{\Delta_{0}, r_{0}}(\widetilde{\phi})=c_{\widetilde{\phi}}\left(\Delta_{0}, r_{0}\right)+\sum_{n \geq 1} q^{n} \sum_{a \mid n}\left(\frac{\Delta_{0}}{a}\right) C_{\widetilde{\phi}}\left(\Delta_{0} \frac{n^{2}}{a^{2}}, r_{0} \frac{n}{a}\right)
$$

for $\Gamma_{0}(m)$ to each $\widetilde{\phi} \in J_{2, m}^{+}$, where $\left(\frac{\Delta_{0}}{a}\right)$ denotes the Jacobi symbol and $c_{\widetilde{\phi}}\left(\Delta_{0}, r_{0}\right)$ denotes a suitably chosen constant term.

From the discussion in the 'The Eichler-Zagier operators and an ADE classification' section, it is not difficult to see that given a simply laced root system $X$ with each of 
its irreducible components having the same Coxeter number $m$, we may consider the following skew-holomorphic form

$$
\begin{aligned}
\sigma^{X} & =\left(\overline{S^{X}}\right)^{T} \cdot \theta_{m} \\
& =\sum_{r(\bmod 2 m)} \overline{S_{r}^{X}(\tau)} \theta_{m, r}(\tau, z) \\
& =\sum_{r, r^{\prime}(\bmod 2 m)} \overline{S_{m, r}(\tau)} \Omega_{r, r^{\prime}}^{X} \theta_{m, r^{\prime}}(\tau, z)
\end{aligned}
$$

of weight 2 and index $m$.

Applying $\mathscr{S}_{\Delta_{0}, r_{0}}$ with the simplest choice $\left(\Delta_{0}, r_{0}\right)=(1,1)$ to the skew-holomorphic Jacobi form $\sigma^{X}$, and using the fact that the Jacobi symbol $\left(\frac{1}{a}\right)=1$ for all positive integers $a$, we arrive at a weight 2 form on $\Gamma_{0}(m)$

$$
\begin{aligned}
f^{X}(\tau) & =\mathscr{S}_{1,1}\left(\sigma^{X}\right)(\tau) \\
& =\frac{r}{24}+\sum_{r=1}^{m-1} \alpha_{r}^{X} \sum_{\varepsilon= \pm 1} \sum_{k=-\left\lfloor\frac{\varepsilon r}{2 m}\right\rfloor}^{\infty}(2 k m+\varepsilon r) \frac{q^{2 k m+\varepsilon r}}{1-q^{2 k m+\varepsilon r}}
\end{aligned}
$$

where $r$ is the rank of the root system $X$ and $\alpha_{r}^{X}$ is the multiplicity of the multiplicity of $r$ as a Coxeter exponent of $X$, which also coincides with the 'diagonal' coefficient $\Omega_{r, r}^{X}-\Omega_{r,-r}^{X}$ of $S_{m, r}$ in the $r$-th component of the vector-valued cusp form $S^{X}=\left(S_{r}^{X}\right)$ (cf. Table 3 and 'The Eichler-Zagier operators and an ADE classification' section).

For $X=A_{m-1}$, one can easily see from the fact that $\alpha_{r}^{X}=1$ for all $r \in\{1,2, \ldots, m-1\}$ that the associated weight 2 form is nothing but the following Eisenstein form at level $m$ $(c f .(237))$

$$
\begin{aligned}
f^{A_{m-1}}(\tau) & =\lambda_{m}(\tau) \\
& =q \frac{d}{d q} \log \left(\frac{\eta(m \tau)}{\eta(\tau)}\right)=\frac{m-1}{24}+\sum_{k>0} \sigma(k)\left(q^{k}-m q^{m k}\right) .
\end{aligned}
$$

One can compute the function $f^{X}$ in a similar way for the D- and E-series and arrive at the result in Table 4. From this table, one observes that the weight 2 form $f^{X}$ has a close relation to the Coxeter Frame shape $\pi^{X}$ and hence also to the matrix $\Omega^{X}$ according to Table 6. We now discuss this further.

From $\Omega^{X}$ where $X$ has Coxeter number $m$, we can obtain a map on the space spanned by the weight 2 modular forms $\left\{\lambda_{n}(\tau), n \mid m\right\}$ of level $m$ by replacing each $\Omega_{m}\left(m_{1}\right)$ in $\Omega^{X}$ with the operator $w_{m}\left(m_{1}\right)$ which acts on Eisenstein forms according to

$$
\lambda_{m_{2}} \mid w_{m}\left(m_{1}\right)=\lambda_{m_{1} * m_{2}}-\lambda_{m_{1}},
$$

where $m_{1}$ and $m_{2}$ are assumed to divide $m$, and $m_{1} * m_{2}=m_{1} m_{2} /\left(m_{1}, m_{2}\right)^{2}(c f$. 'Genus zero groups' section). Then, the weight 2 form corresponding to a simply laced root system $X$ with Coxeter number $m$ is nothing but

$$
f^{X}=\lambda_{m} \mid w^{X}=\lambda_{\pi^{X}}
$$

where $\pi^{X}$ is the Coxeter Frame shape of $X$ ( $c f$. 'Root systems' section) and $\lambda_{\pi}$, for $\pi$ an arbitrary Frame shape, is defined in the 'Genus zero groups' section.

The reader will notice that the map $w_{m}(e)$ acts in the same way as the Atkin-Lehner involution $W_{m}(e)(20)$ on modular forms for $\Gamma_{0}(m)$ in the cases where $e \| m$. (Notice that 
the set $W_{m}(e)$ is empty if $e$ is not an exact divisor of $\left.m\right)$. Indeed, from the definition of the Eisenstein form (237), one can compute that

$$
\begin{aligned}
\left(\lambda_{f} \mid W_{m}(e)\right)(\tau) & =\left(\left.\lambda_{f}\right|_{\psi=1, w=2} \gamma\right)(\tau) \\
& =e(c m \tau+d e)^{-2} \lambda_{f}\left(\frac{a e \tau+b}{c m \tau+d e}\right)=\lambda_{e * f}(\tau)-\lambda_{e}(\tau),
\end{aligned}
$$

where $\gamma=\frac{1}{\sqrt{e}}\left(\begin{array}{cc}a e & b \\ c m & d e\end{array}\right) \in S L_{2}(\mathbb{R})(c f .(51))$. On the other hand, from (71), we see that the skew-holomorphic form $\sigma^{X}$ can be obtained as the image of the Eichler-Zagier operator

$$
\sigma^{X}=\sigma^{A_{m-1}} \mid \mathcal{W}^{X} .
$$

Taken together, at a given $m$ and for a given union $X$ of simply laced root systems with Coxeter number $m$, we have the equality

$$
f^{X}=\mathscr{S}_{1,1}\left(\sigma^{A_{m-1}} \mid \mathcal{W}^{X}\right)=\sum_{i} \mathscr{S}_{1,1}\left(\sigma^{A_{m-1}} \mid \mathcal{W}_{m}\left(e_{i}\right)\right)
$$

where we have written $\mathcal{W}^{X}=\sum_{i} \mathcal{W}_{m}\left(e_{i}\right)$ explicitly in terms of its different components. At the same time, for the cases that all $e_{i} \| m$, we also have

$$
f^{X}=\sum_{i} f^{A_{m-1}} \mid W_{m}\left(e_{i}\right)
$$

For these cases, we note that the equality $f^{A_{m-1}} \mid W_{m}\left(e_{i}\right)=\mathscr{S}_{1,1}\left(\sigma^{A_{m-1}} \mid \mathcal{W}_{m}\left(e_{i}\right)\right)$ can be viewed as a consequence of the relation between the Eichler-Zagier operators and the Atkin-Lehner involutions observed in [44,45]. Due to this relation, the Eichler-Zagier operators that define involutions are sometimes referred to as Atkin-Lehner involutions on Jacobi forms in the literature.

We conclude this section by observing that if $X$ is a Niemeier root system, then

$$
\mathscr{S}_{1,1}\left(\sigma^{X}\right)=f^{X}=-q \frac{d}{d q} \log T^{X}
$$

where $\sigma^{X}$ is the skew-holomorphic Jacobi form defined by $S^{X}$ in (126) and $T^{X}$ is the principal modulus for $\Gamma^{X}$ defined in the 'Genus zero groups' section. In this way, we obtain a direct connection between the umbral shadows $S^{X}$ and the genus zero groups $\Gamma^{X}$ attached to Niemeier root systems in the 'Genus zero groups' section.

\section{From weight 0 Jacobi forms to umbral mock modular forms}

The goal of this subsection is to construct the umbral mock modular form $H^{X}$ which (conjecturally) encodes the graded dimension of the umbral module $K^{X}$ ( $c f$. 'Modules' section) for every A-type Niemeier root system $X$. In the 'The umbral shadows' section, we have seen how to associate an umbral shadow $S^{X}$ to a Niemeier root system $X$. Equivalently, we can associate a pole structure, which together with the optimal growth condition (121) determines (at most one) meromorphic weight 1 index $m$ Jacobi form $\psi^{X}$ according to Theorem 2 . In this subsection, we will explicitly construct meromorphic Jacobi forms $\psi^{X}$ satisfying the conditions of Theorem 2 for $X$ an A-type root system via certain weight 0 Jacobi forms $\phi^{X}$ (cf. Proposition 5). After obtaining these $\psi^{X}$, the procedure discussed in the 'From Meromorphic Jacobi forms to mock modular forms' section then immediately leads to the umbral forms $H^{X}$. It is also possible to specify the D- and E-type Niemeier root systems in a similar way. However, the discussion would become somewhat less illuminating, and we will instead specify these in an arguably more elegant way in the 'The umbral McKay-Thompson series' section. 
Our strategy in the present subsection is the following. As mentioned in the "The umbral shadows' section, if we take a weight 0 index $m-1$ weak Jacobi form $\phi$ with $\phi(\tau, 0)=1$, then $-\mu_{1,0} \phi \mid \mathcal{W}^{X}$ is a weight 1 index $m$ meromorphic Jacobi whose pole structure is automatically of the desired form (cf. (89)). Namely, it always leads to a vectorvalued mock modular form whose shadow is given by $S^{X}$. In this subsection, we will see how to select (uniquely) a weight 0 form $\phi^{X}$ such that the resulting weight 1 Jacobi form

$$
\psi^{X}=-\mu_{1,0} \phi^{X} \mid \mathcal{W}^{X}
$$

satisfies the optimal growth condition (121).

For the simplest cases, this optimal growth condition can be rephrased in terms of weight 0 Jacobi forms using the language of characters of the $\mathcal{N}=4$ superconformal algebra. Recall from $[7,8]$ that this algebra contains subalgebras isomorphic to the affine Lie algebra $\widehat{A}_{1}$ as well as the Virasoro algebra, and in a unitary representation, the former of these acts with level $m-1$, for some integer $m>1$, and the latter with central charge $c=6(m-1)$. The unitary irreducible highest weight representations $V_{h, j}^{(m)}$ are labelled by the two 'quantum numbers' $h$ and $j$ which are the eigenvalues of $L_{0}$ and $\frac{1}{2} J_{0}^{3}$, respectively, when acting on the highest weight state. (We adopt a normalisation of the $S U$ (2) current $J^{3}$ such that the zero mode $J_{0}^{3}$ has integer eigenvalues). In the Ramond sector of the superconformal algebra, there are two types of highest weight representations: the short (or BPS, supersymmetric) ones with $h=\frac{m-1}{4}$ and $j \in\left\{0, \frac{1}{2}, \cdots, \frac{m-1}{2}\right\}$, and the long (or nonBPS, non-supersymmetric) ones with $h>\frac{m-1}{4}$ and $j \in\left\{\frac{1}{2}, 1, \cdots, \frac{m-1}{2}\right\}$. Their (Ramond) characters, defined as

$$
\operatorname{ch}_{h, j}^{(m)}(\tau, z)=\operatorname{tr}_{V_{h, j}^{(m)}}\left((-1)^{3} y_{0}^{3} q^{3} q^{L_{0}-c / 24}\right),
$$

are given by

$$
\operatorname{ch}_{\frac{m-1}{4}, j}^{(m)}(\tau, z)=\frac{\mu_{m, j}(\tau, z)}{\mu_{1,0}(\tau, z)}
$$

and

$$
\operatorname{ch}_{h, j}^{(m)}(\tau, z)=(-1)^{2 j} q^{h-\frac{m-1}{4}-\frac{j^{2}}{m}} \frac{\theta_{m, 2 j}(\tau, z)-\theta_{m,-2 j}(\tau, z)}{\mu_{1,0}(\tau, z)}
$$

in the short and long cases, respectively, [8], where the function $\mu_{m, j}(\tau, z)$ is defined as in (83).

\section{Lambencies 2,3,4,5,7,13}

It turns out that for the pure A-type Niemeier root systems given as the union of $24 /(\ell-1)$ copies of $A_{\ell-1}$ for $(\ell-1) \mid 12$, the relevant criterion for $\phi^{(\ell)}$ is that of an extremal Jacobi form ([1], Section 2.5). The idea of an extremal Jacobi form can be viewed as a generalisation of the concept of an extremal Virasoro character, a notion that was introduced in [47] and discussed in [48] in the context of pure $A d S_{3}$ gravity.

With the above definitions, following [1], for $m$ a positive integer and $\phi$ a weak Jacobi form with weight 0 and index $m-1$, we say $\phi$ is extremal if it admits an expression

$$
\begin{aligned}
\phi= & a_{\frac{m-1}{4}, 0} \operatorname{ch}_{\frac{m-1}{4}, 0}^{(m)}+a_{\frac{m-1}{4}, \frac{1}{2}} \operatorname{ch}_{\frac{m-1}{4}, \frac{1}{2}}^{(m)} \\
& +\sum_{0<r<m} \sum_{\substack{n>0 \\
r^{2}-4 m n<0}} a_{\frac{m-1}{4}+n, \frac{r}{2}} \operatorname{ch}_{\frac{m-1}{4}+n, \frac{r}{2}}^{(m)}
\end{aligned}
$$


for some $a_{h, j} \in \mathbb{C}$. Note the restriction on $n$ in the last summation in (140). Write $J_{0, m-1}^{\mathrm{ext}}$ for the subspace of $J_{0, m-1}$ consisting of extremal weak Jacobi forms.

We recall here that the extremal condition has a very natural interpretation in terms of the mock modular forms $h=\left(h_{r}\right)$ of weight $1 / 2$ via the relation

$$
\psi=-\mu_{1,0} \phi, \quad \psi^{F}=\sum_{r(\bmod 2 m)} h_{r} \theta_{m, r}
$$

discussed in the 'The umbral shadows' section. More precisely, the extremal condition is equivalent to the condition that

$$
h_{r}=r \delta_{r^{2}, 1} a_{\frac{m-1}{4}, \frac{1}{2}} q^{-\frac{1}{4 m}}+O\left(q^{\frac{1}{4 m}}\right)
$$

as $\tau \rightarrow i \infty$, which clearly implies the optimal growth condition $q^{1 / 4 m} h_{r}(\tau)=O(1)$ of Theorem 2.

In [1], we proved that $\operatorname{dim} J_{0, m-1}^{\mathrm{ext}}=1$ in case $m-1$ divides 12 , and vanishes otherwise, at least when $m \leq 25$. (cf. [1], Section 2.5). Explicitly, if $m-1$ divides 12, then the one-dimensional space $J_{0, m-1}^{\text {ext }}$ of extremal Jacobi forms with index $m-1$ is spanned by $\varphi_{1}^{(m)}$, using the basis defined in 'Weight 0 Jacobi forms' in Appendix 1. These weight 0 forms $\varphi_{1}^{(m)}$ arising from the extremal condition (140) will determine the unique weight 1 meromorphic Jacobi forms $\psi^{X}$ satisfying the conditions of Theorem 2 for $X \in\left\{A_{1}^{24}, A_{2}^{12}, A_{3}^{8}, A_{4}^{6}, A_{6}^{4}, A_{12}^{2}\right\}$ according to (136) where $m$ is the Coxeter number of $X$.

\section{Lambencies 9,25}

In order to include the other two pure A-type Niemeier root systems $\left(X=A_{8}^{3}\right.$ and $\left.X=A_{24}\right)$, it is sufficient to weaken the extremal condition slightly and consider weight 0 and index $m-1$ Jacobi forms admitting an expression

$$
\begin{aligned}
\phi= & a_{\frac{m-1}{4}, 0} \operatorname{ch}_{\frac{m-1}{4}, 0}^{(m)}+a_{\frac{m-1}{4}, \frac{1}{2}} \mathrm{ch}_{\frac{m-1}{4}, \frac{1}{2}}^{(m)} \\
& +\sum_{0<r<m} \sum_{\substack{n>0 \\
r^{2}-4 m n \leq 0}} a_{\frac{m-1}{4}+n, \frac{r}{2}} \operatorname{ch}_{\frac{m-1}{4}+n, \frac{r}{2}}^{(m)}
\end{aligned}
$$

for some $a_{h, j} \in \mathbb{C}$. Notice that we have weakened the bound from $r^{2}-4 m n<0$ to $r^{2}-4 m n \leq 0$ in the last summand. This is directly related to the fact that for $m=9,25$, there exists $0<r<m$ such that $r^{2}=0(\bmod 4 m)$.

There exists at most one solution to (143) up to rescaling (look ahead to Lemma 3 ), and inspection reveals that at $m=9,25$, we have the non-zero solutions $\varphi_{1}^{(9)}$ and $\varphi_{1}^{(25)}$, in the notation of 'Weight 0 Jacobi forms' in Appendix 1. These Jacobi forms will determine, by way of (136), the unique weight 1 meromorphic Jacobi forms $\psi^{X}$ satisfying the conditions of Theorem 2 for $X \in\left\{A_{8}^{3}, A_{24}\right\}$.

\section{Lambencies 6,10}

In order to capture $X=A_{5}^{4} D_{4}$ and $X=A_{9}^{2} D_{6}$ at $m=6$ and $m=10$ (the cases with $m$ given by the product of two distinct primes), we will relax the extremal condition further and consider weight 0 and index $m-1$ Jacobi forms admitting an expression

$$
\begin{aligned}
\phi= & a_{\frac{m-1}{4}, 0} \operatorname{ch}_{\frac{m-1}{4}, 0}^{(m)}+a_{\frac{m-1}{4}, \frac{1}{2}} \operatorname{ch}_{\frac{m-1}{4}, \frac{1}{2}}^{(m)} \\
& +\sum_{0<r<m} \sum_{\substack{n>0 \\
r^{2}-4 m n \leq 1}} a_{\frac{m-1}{4}+n, \frac{r}{2}} \operatorname{ch}_{\frac{m-1}{4}+n, \frac{r}{2}}^{(m)}
\end{aligned}
$$


for some $a_{h, j} \in \mathbb{C}$. For this more general condition, we also have a uniqueness property.

Proposition 3. For a given index $m-1$, the dimension of the space of Jacobi forms satisfying (144) for some $a_{h, j} \in \mathbb{C}$ is at most 1 .

Proof. If $\phi_{1}$ and $\phi_{2}$ are two weight 0 Jacobi forms that can be written as in (144), there exists a linear combination $\phi$ of $\phi_{1}$ and $\phi_{2}$ satisfying

$$
\phi=a q^{-\frac{1}{4 m}} \frac{\theta_{m, 1}-\theta_{m,-1}}{\mu_{1,0}}+\sum_{0<r<m} \sum_{\substack{n>0 \\ r^{2}-4 m n \leq 1}} a_{\frac{m-1}{4}+n, \frac{r}{2}} \operatorname{ch}_{\frac{m-1}{4}+n, \frac{r}{2}}^{(m)}
$$

for some $a, a_{h, j} \in \mathbb{C}$, where we have used that

$$
2 \operatorname{ch}_{\frac{m-1}{4}, 0}^{(m)}+\operatorname{ch}_{\frac{m-1}{4}, \frac{1}{2}}^{(m)}+q^{-\frac{1}{4 m}} \frac{\theta_{m, 1}-\theta_{m,-1}}{\mu_{1,0}}=0 .
$$

Equivalently, $\psi=\mu_{1,0} \phi$ is a weight 1 index $m$ weak Jacobi form with Fourier expansion $\psi(\tau, z)=\sum_{n, \ell} c(n, \ell) q^{n} y^{\ell}$, where $c(n, \ell)=0$ for $\ell^{2}-4 m n>1$. But such a weight 1 index $m$ weak Jacobi form does not exist according to Theorem 9.7 of [41], based on the fact that there is no (strong) Jacobi form of weight 1 and index $m$ for any positive integer $m$, as shown earlier by Skoruppa [49]. We therefore conclude that $\phi=0$, and $\phi_{1}$ and $\phi_{2}$ are linearly dependent.

This more general condition (144) singles out the weight 0 Jacobi forms $\varphi_{1}^{(6)}$ and $\varphi_{1}^{(10)}$ (cf. 'Weight 0 Jacobi forms' in Appendix 1 ) at $m \in\{6,10\}$ in addition to those already mentioned, for which $m$ is prime or the square of a prime. As above, these weight 0 forms will determine, by way of (136), the unique weight 1 meromorphic Jacobi forms $\psi^{X}$ satisfying the conditions of Theorem 2 for $X \in\left\{A_{5}^{4} D_{6}, A_{9}^{2} D_{6}\right\}$.

Remark 3. We expect that the space of solutions to (144) is zero-dimensional for all but finitely many $m$. The discussion of weight 1 Jacobi forms in [41], Section 9, suggests that Jacobi forms satisfying (144) might only exist for these values $m$ $\in\{2,3,4,5,6,7,9,10,13,25\}$ which are among those of relevance to umbral moonshine.

\section{Lambencies 8,12,16,18}

We are left with the A-type Niemeier root systems with Coxeter numbers that are not square-free and not squares of primes: they are

$$
X=A_{7}^{2} D_{5}^{2}, A_{11} D_{7} E_{6}, A_{15} D_{9}, A_{17} E_{7}
$$

with $m=8,12,16,18$, respectively. To discuss these cases, let us first point out a subtlety in our procedure for determining the weight 1 meromorphic Jacobi form $\psi^{X}$ from a weight 0 meromorphic Jacobi form $\phi^{X}$ using (136). Although the resulting weight 1 form $\psi^{X}$ is unique following Theorem 2, in general, the corresponding weight 0 form $\phi^{X}$ is not. In other words, there could be more than one $\phi^{X}$ satisfying (136) for a given $\psi^{X}$. For the A-type cases with $m \in\{2,3,4,5,6,7,9,10,13,25\}$ discussed above, there is no such ambiguity since the matrix $\Omega^{X}$ corresponding to the Eichler-Zagier operator $\mathcal{W}^{X}$ is invertible. On the other hand, the matrix $\Omega^{X}$ is not invertible for $X$ $\in\left\{A_{7}^{2} D_{5}^{2}, A_{11} D_{7} E_{6}, A_{15} D_{9}, A_{17} E_{7}\right\}$, corresponding to $m \in\{8,12,16,18\}$. At the same time, in these cases, there exists a unique $d>1$ such that $d^{2}$ is a proper divisor of $m$, and correspondingly, there is an interesting feature in the space of Jacobi forms. This is due to the 
fact (cf. [41], Section 4.4) that if $\psi_{k, m / d^{2}}(\tau, z)$ transforms as a weight $k$ index $m / d^{2}$ Jacobi form, then $\psi_{k, m / d^{2}}(\tau, d z)$ transforms as a weight $k$ index $m$ Jacobi form. It turns out that the umbral forms discussed above, in particular those with lambency $\ell=2,3,4$, help to determine the weight 0 forms $\phi^{X}$ at lambency $\ell=8,12,16,18$ by requiring the 'square relation'

$$
-\mu_{1,0} \phi^{(\ell)} \mid \mathcal{W}_{\ell}(d)(\tau, z)=\frac{\ell / d^{2}-1}{24} \psi^{\left(\ell / d^{2}\right)}(\tau, d z),
$$

where $d$ is the unique integer such that $d^{2}$ is a proper divisor of $\ell$ different from 1 , and $\psi^{\left(\ell / d^{2}\right)}$ is the weight 1 meromorphic Jacobi form with index $\ell / d^{2}$ that we have constructed above via (136). This extra condition (147) eliminates the kernel of the Eichler-Zagier operator $\mathcal{W}^{X}$ and renders our choice for $\phi^{X}$ unique. Notice that, following Table 2, we have used the lambency $\ell$ to denote the Niemeier root system $X$, and the former simply coincides with the Coxeter number for the A-type cases discussed in this subsection.

To specify this particular choice of $\phi^{X}$, let us impose the following condition. For a non-square-free $m$ which is not a square of a prime, we consider the weight 0 index $m-1$ Jacobi forms $\phi$, such that the finite part of the weight 1 index $m$ Jacobi form $-\mu_{1,0} \phi \mid\left(\mathbf{1}+\mathcal{W}_{m}(m / d)\right)$ is a mock Jacobi form with expansion $\sum_{n, \ell} c(n, \ell) q^{n} y^{\ell}$ and $c(n, r)=0$ whenever $r^{2}-4 m n>1$, i.e.

$$
\left(-\mu_{1,0} \phi \mid\left(\mathbf{1}+\mathcal{W}_{m}(m / d)\right)\right)^{F}(\tau, z)=\sum_{\substack{n, r \in \mathbb{Z}, n \geq 0 \\ r^{2}-4 m n \leq 1}} c(n, r) q^{n} y^{r} .
$$

From the above discussion, we arrive at the following uniqueness property for such Jacobi forms.

Proposition 4. Consider integers $m, \tilde{m}, d$ satisfying $m=\widetilde{m} d^{2}$ and $\widetilde{m}, d>1$. Given any meromorphic weight 1 index $n$ Jacobi form $\psi^{(\widetilde{m})}(\tau, z)$, there exists at most one weight 0 index $m-1$ weak Jacobi form $\phi$ satisfying (148) and the square relation

$$
-\mu_{1,0} \phi \mid \mathcal{W}_{m}(d)(\tau, z)=\psi^{(\widetilde{m})}(\tau, d z) .
$$

Proof. Assume that there are two weight 0 index $m-1$ weak Jacobi forms $\phi_{1}$ and $\phi_{2}$ satisfying

$$
\mu_{1,0} \phi_{1}\left|\mathcal{W}_{m}(d)(\tau, z)=\mu_{1,0} \phi_{2}\right| \mathcal{W}_{m}(d)(\tau, z)=\psi^{(\widetilde{m})}(\tau, d z)
$$

and therefore $\left(\mu_{1,0} \phi_{1} \mid \mathcal{W}_{m}(d)\right)^{F}=\left(\mu_{1,0} \phi_{2} \mid \mathcal{W}_{m}(d)\right)^{F}$. It then follows from Lemma 1 that

$$
\left(\mu_{1,0} \phi_{1} \mid \mathcal{W}_{m}(m / d)\right)^{F}=\left(\mu_{1,0} \phi_{2} \mid \mathcal{W}_{m}(m / d)\right)^{F} .
$$

Note that we also have

$$
\begin{aligned}
\phi_{1}(\tau, 0) \mu_{m, 0} \mid \mathcal{W}_{m}(d) & =\left(\mu_{1,0} \phi_{1} \mid \mathcal{W}_{m}(d)\right)^{P} \\
& =\left(\mu_{1,0} \phi_{2} \mid \mathcal{W}_{m}(d)\right)^{P} \\
& =\phi_{2}(\tau, 0) \mu_{m, 0} \mid \mathcal{W}_{m}(d),
\end{aligned}
$$

which leads to the equality between the two constants $\phi_{1}(\tau, 0)=\phi_{2}(\tau, 0)$, and hence

$$
\left(\mu_{1,0} \phi_{1} \mid\left(\mathbf{1}+\mathcal{W}_{m}(m / d)\right)\right)^{P}=\left(\mu_{1,0} \phi_{2} \mid\left(\mathbf{1}+\mathcal{W}_{m}(m / d)\right)\right)^{P} .
$$


Next, assume that $\phi_{1}$ and $\phi_{2}$ both satisfy (148). Then, $\mu_{1,0}\left(\phi_{1}-\phi_{2}\right) \mid\left(\mathbf{1}+\mathcal{W}_{m}(m / d)\right)$ is a weight 1 index $m$ weak Jacobi form with expansion $\sum_{n, \ell} c(n, \ell) q^{n} y^{\ell}$, where $c(n, \ell)=0$ for $\ell^{2}-4 m n>1$, which can only be identically zero (cf. [41], Theorem 9.7), and we arrive at

$$
\mu_{1,0} \phi_{1}\left|\left(\mathbf{1}+\mathcal{W}_{m}(m / d)\right)=\mu_{1,0} \phi_{2}\right|\left(\mathbf{1}+\mathcal{W}_{m}(m / d)\right) .
$$

Combining with (151) and using Corollary 1 , we obtain $\left(\mu_{1,0} \phi_{1}\right)^{F}=\left(\mu_{1,0} \phi_{2}\right)^{F}$. Again, the polar part $\left(\mu_{1,0} \phi\right)^{P}$ of the meromorphic Jacobi form $\mu_{1,0} \phi$ for any weight 0 index $m-1$ weak Jacobi form $\phi$ is given by $\phi(\tau, 0) \mu_{m, 0}$. This proves that $\mu_{1,0} \phi_{1}=\mu_{1,0} \phi_{2}$ and hence $\phi_{1}=\phi_{2}$.

At $m \in\{8,12,16,18\}$, applying the above proposition and choosing

$$
\psi^{(\widetilde{m})}=-\mu_{1,0} \phi^{(\widetilde{m})}, \quad \widetilde{m}=2,3,4,
$$

with $\phi^{(\widetilde{m})}$ as in Table 7 gives the weight 0 forms we need in order to specify the remaining umbral mock modular forms $H^{X}$ for $X$ of A-type.

The explicit expressions for $\phi^{X}$ for all A-type Niemeier root systems $X$ are given in Table 7, where the basis we use for weight 0 weak Jacobi forms is summarised in 'Weight 0 Jacobi forms' in Appendix 1 and $\varphi_{0}^{(\ell)}$ denotes the constant $\varphi_{0}^{(\ell)}=\varphi_{1}^{(\ell)}(\tau, 0)$.

Given $\phi^{X}$ and using the Eichler-Zagier operator $\mathcal{W}^{X}$ defined in the 'The EichlerZagier operators and an ADE classification' section, the formula (136) gives the weight 1 meromorphic Jacobi form $\psi^{X}$. From there, using the method described in the 'From Meromorphic Jacobi forms to mock modular forms' section, we can separate it into the polar and the finite part $\psi^{X}=\left(\psi^{X}\right)^{P}+\left(\psi^{X}\right)^{F}$ in a canonical way. As can be verified by inspection, the choices of $\phi^{X}$ specified in this subsection determine solutions to the hypotheses of Theorem 2.

Proposition 5. Let $X$ be one of the 14 Niemeier root systems with an A-type component (cf. (11)) and let $\phi^{X}$ be as specified in Table 7. Then, the meromorphic Jacobi form $\psi^{X}$ determined by (136) is the unique such function satisfying the conditions of Theorem 2. Write

$$
\left(\psi^{X}\right)^{F}=\sum_{r(\bmod 2 m)} H_{r}^{X} \theta_{m, r}
$$

then $H^{X}=\left(H_{r}^{X}\right)$ is the unique vector-valued mock modular form with shadow $S^{X}$ satisfying the optimal growth condition (cf. Corollary 2).

As a result, the weight 0 weak Jacobi forms constructed in this subsection define the umbral mock modular forms $H^{X}$ for each of the A-type Niemeier root systems (cf. (11)). The first few dozen coefficients of the components $H_{r}^{X}$ are given in Additional file 1. It is a reflection of the close relationship between the notions of optimal growth formulated here and in [41] that the umbral forms $H^{X}$ attached to A-type Niemeier root systems are closely related to the mock modular forms of weight 1 that appear in Section A.2 of [41].

\section{The umbral McKay-Thompson series}

The purpose of this section is to discuss the umbral McKay-Thompson series $H_{g}^{X}$ conjecturally defining the graded character of the group $G^{X}$ attached to the umbral module $K^{X}$ (cf. 'Modules' section). In the 'Shadows' section, we specify their mock modular properties. In the 'Prime lambencies' section, we discuss the McKay-Thompson series $H_{g}^{X}$ 
Table 7 Weight zero Jacobi forms

\begin{tabular}{cccccccccccc}
\hline$x$ & $A_{1}^{24}$ & $A_{2}^{12}$ & $A_{3}^{8}$ & $A_{4}^{6}$ & $A_{5}^{4} D_{4}$ & $A_{6}^{4}$ & $A_{8}^{3}$ & $A_{9}^{2} D_{6}$ & $A_{12}^{2}$ & $A_{24}$ \\
\hline$\ell$ & 2 & 3 & 4 & 5 & 6 & 7 & 9 & 10 & 13 & 25 \\
\hline$\varphi_{0}^{(\ell)} \phi^{(\ell)}$ & $\varphi_{1}^{(2)}$ & $\varphi_{1}^{(3)}$ & $\varphi_{1}^{(4)}$ & $\varphi_{1}^{(5)}$ & $\varphi_{1}^{(6)}$ & $\varphi_{1}^{(7)}$ & $\varphi_{1}^{(9)}$ & $\varphi_{1}^{(10)}$ & $\varphi_{1}^{(13)}$ & $\varphi_{1}^{(25)}$ \\
$X$ & $A_{7}^{2} D_{5}^{2}$ & $A_{11} D_{7} E_{6}$ & $A_{15} D_{9}$ & & & \\
\hline$\ell$ & 8 & 12 & 16 & & \\
\hline$\varphi_{0}^{(\ell)} \phi^{(\ell)}$ & $\varphi_{1}^{(8)}+\frac{1}{2} \varphi_{2}^{(8)}$ & $\varphi_{1}^{(12)}+\varphi_{2}^{(12)}$ & $\varphi_{1}^{(16)}+\frac{1}{2} \varphi_{2}^{(16)}$ & & \\
$X$ & $A_{17} E_{7}$ & & & \\
\hline$\ell$ & 18 & & & \\
\hline$\varphi_{0}^{(\ell)} \phi^{(\ell)}$ & $\varphi_{1}^{(18)}+\frac{1}{3} \varphi_{3}^{(18)}+4 \xi\left(\varphi_{1}^{(12)}+2 \varphi_{2}^{(12)}+\frac{1}{3} \varphi_{3}^{(12)}\right)$ & & \\
\hline
\end{tabular}


attached to the five A-type Niemeier root systems $X$ with prime Coxeter numbers. Subsequently, in the 'Multiplicative relations' section, we discuss the multiplicative relations relating the McKay-Thompson series $H_{g}^{X}$ and $H_{g^{\prime}}^{X^{\prime}}$ attached to different Niemeier root systems $X$ and $X^{\prime}$ with the Coxeter number of one of the root systems being an integer multiple of the other. In the 'Mock theta functions' section, we collect the relations between certain McKay-Thompson series and known mock theta series. As we discuss in the 'Specification' section in detail, these relations, together with the constructions presented in the 'From weight 0 Jacobi forms to umbral mock modular forms' and 'Prime lambencies' sections, are sufficient to determine most of the umbral McKay-Thompson series completely. More specifically, we determine all of the umbral McKay-Thompson series $H_{g}^{X}$ attached to all conjugacy classes $[g]$ of the umbral group $G^{X}$ corresponding to all the A-type Niemeier root systems, except for $X=A_{6}^{4}, A_{12}^{2}$, corresponding to lambencies 7,13 (cf. 'Genus zero groups' section), for which we provide partial specifications. We also determine all of the umbral McKay-Thompson series $H_{g}^{X}$ for all conjugacy classes $[g]$ of the umbral group $G^{X}$ corresponding to all the D-type Niemeier root systems, except for the lambencies $10+5$ and $22+11$. For these D-type Niemeier root systems $10+5$ and $22+11$, we specify all the McKay-Thompson series $H_{g}^{X}$ except for $[g]=4 A \subset G^{(10+5)}$ and $[g]=2 A \subset G^{(22+11)}$. For $X=E_{6}^{4}$, we specify $H_{g}^{X}$ except for $[g] \in\{6 A, 8 A B\}$, while for $X=E_{8}^{3}$, we omit the cases $[g] \in\{2 A, 3 A\}$. We also provide the first few dozen coefficients of all the umbral McKay-Thompson series in Additional file 1 . The conjugacy class names are defined in 'Irreducible characters' in Appendix 2.

\section{Shadows}

In the 'Umbral groups' section, we described the umbral groups $G^{X}$ and attached twisted Euler characters $\bar{\chi}^{X_{A}}, \chi^{X_{A}}, \bar{\chi}^{X_{D}}, \chi^{X_{D}}$, \&c., to the A-, D- and E-components of each Niemeier root system. In this section, we will explain how to use these characters to define a function $S_{g}^{X}$, for each $g \in G^{X}$, which turns out to be the shadow of the vector-valued mock modular form $H_{g}^{X}$.

Let $X$ be a Niemeier root system and suppose that $m$ is the Coxeter number of $X$. Then, given $g \in G^{X}$, we define $2 m \times 2 m$ matrices $\Omega_{g}^{X_{A}}, \Omega_{g}^{X_{D}}$ and $\Omega_{g}^{X_{E}}$, with entries indexed by $\mathbb{Z} / 2 m \mathbb{Z} \times \mathbb{Z} / 2 m \mathbb{Z}$, as follows. We define $\Omega_{g}^{X_{A}}$ by setting

$$
\Omega_{g}^{X_{A}}=\chi_{g}^{X_{A}} P_{m}^{0}+\bar{\chi}_{g}^{X_{A}} P_{m}^{1}
$$

where $P_{m}^{s}$ is the diagonal matrix (of size $2 m \times 2 m$, with entries indexed by $\mathbb{Z} / 2 m \mathbb{Z} \times$ $\mathbb{Z} / 2 m \mathbb{Z})$ with $r$-th diagonal equal to 1 or 0 according as $r=s(\bmod 2)$ or not,

$$
\left(P_{m}^{s}\right)_{r, r^{\prime}}=\delta_{r, s(2)} \delta_{r, r^{\prime}(2 m)}
$$

In (158), we write $\delta_{i, j(n)}$ for the function that is 1 when $i=j(\bmod n)$ and 0 otherwise. Note that $P_{m}^{0}+P_{m}^{1}=\Omega_{m}(1)$ is the $2 m \times 2 m$ identity matrix. According to the convention that $\chi_{g}^{X_{A}}=\bar{\chi}^{X_{A}}=0$ if $X_{A}=\emptyset$, we have $\Omega_{g}^{X_{A}}=0$ for all $g \in G^{X}$ in case $X_{A}$ is empty, i.e. in case there are no type A components in the Niemeier root system $X$.

If $X_{D} \neq \emptyset$, then $m$ is even. For $m>6$, we define $\Omega_{g}^{X_{D}}$ by setting

$$
\Omega_{g}^{X_{D}}=\chi_{g}^{X_{D}} P_{m}^{0}+\bar{\chi}_{g}^{X_{D}} P_{m}^{1}+\chi_{g}^{X_{D}} \Omega_{m}(m / 2),
$$


whilst for $m=6$ - an exceptional case due to triality for $D_{4}$ - we define $\Omega=\Omega_{g}^{X_{D}}$ so that

$$
\Omega_{r, r^{\prime}}= \begin{cases}\check{\chi}_{g}^{X_{D}} \delta_{r, r^{\prime}(12)}, & \text { if } r=0,3(\bmod 6), \\ \bar{\chi}_{g}^{X_{D}} \delta_{r, r^{\prime}(12)}+\chi_{g}^{X_{D}} \delta_{r,-r^{\prime}(6)} \delta_{r, r^{\prime}(4)}, & \text { if } r=1,5(\bmod 6) \\ \chi_{g}^{X_{D}} \delta_{r, r^{\prime}(12)}+\chi_{g}^{X_{D}} \delta_{r,-r^{\prime}(6)} \delta_{r, r^{\prime}(4)}, & \text { if } r=2,4(\bmod 6)\end{cases}
$$

The matrices $\Omega_{g}^{X_{E}}$ are defined by setting

$$
\Omega_{g}^{X_{E}}= \begin{cases}\left(\chi_{g}^{X_{E}} P_{m}^{0}+\bar{\chi}_{g}^{X_{E}} P_{m}^{1}\right)\left(\Omega_{m}(1)+\Omega_{m}(4)\right)+\chi_{g}^{X_{E}} \Omega_{m}(6) & \text { if } m=12, \\ \bar{\chi}_{g}^{X_{E}}\left(\Omega_{m}(1)+\Omega_{m}(6)+\Omega_{m}(9)\right), & \text { if } m=18, \\ \bar{\chi}_{g}^{X_{E}}\left(\Omega_{m}(1)+\Omega_{m}(6)+\Omega_{m}(10)+\Omega_{m}(15)\right), & \text { if } m=30 .\end{cases}
$$

Now for $X$ a Niemeier root system, we set $\Omega_{g}^{X}=\Omega_{g}^{X_{A}}+\Omega_{g}^{X_{D}}+\Omega_{g}^{X_{E}}$, and we define $S_{g}^{X}$ by setting

$$
S_{g}^{X}=\Omega_{g}^{X} \cdot S_{m}
$$

This generalises the construction (118). We conjecture (cf. 'Modularity' section) that the vector-valued function $S_{g}^{X}$ is the shadow of the mock modular form $H_{g}^{X}$ attached to $g \in G^{X}$. We will specify (most of) the $H_{g}^{X}$ explicitly in the remainder of the 'The umbral McKay-Thompson series' section.

Remark 4. The matrices $\Omega^{X}$, corresponding to the case where $[g]$ is the identity class, admit an ADE classification as explained in the 'The Eichler-Zagier operators and an ADE classification' section. It is natural to ask what the criteria are that characterise these matrices $\Omega_{g}^{X}$, attached as above to elements $g \in G^{X}$ via the twisted Euler characters defined in the 'Umbral groups' section.

\section{Prime lambencies}

In this subsection, we review the mock modular forms $H_{g}^{X}=\left(H_{g, r}^{X}\right)$ conjecturally encoding the graded characters of the umbral module $K^{X}$ ( $c f$. Conjecture 1$)$ of the umbral group $G^{X}$ for the five Niemeier root systems $X$ with prime Coxeter numbers. Explicitly, these are the root systems $X=A_{1}^{24}, A_{2}^{12}, A_{4}^{6}, A_{6}^{4}, A_{12}^{2}$ with $\ell=2,3,5,7,13$, and the corresponding McKay-Thompson series are denoted $H_{g}^{(\ell)}=\left(H_{g, r}^{(\ell)}\right)$ with $r=1,2, \ldots, \ell-1$, using the notation given in Table 2 . In the next subsection, we will see that they determine many of the umbral McKay-Thompson series attached to the other Niemeier root systems with non-prime Coxeter numbers. The discussion of this subsection follows that of [1]. The McKay-Thompson series for $\ell=2, X=A_{1}^{24}$ were first computed in [12-15].

In order to give explicit formulas for the mock modular forms $H_{g}^{(\ell)}=\left(H_{g, r}^{(\ell)}\right)$, we consider a slightly different function

$$
\widehat{H}_{g, r}^{(\ell)}=H_{g, r}^{(\ell)}-\frac{\chi_{r}^{(\ell)}(g)}{\chi^{(\ell)}} H_{r}^{(\ell)},
$$

where $H^{(\ell)}=\left(H_{r}^{(\ell)}\right)$ is the umbral form specified in the 'From weight 0 Jacobi forms to umbral mock modular forms' section corresponding to the identity class $1 A$ and whose Fourier coefficients are given in Additional file 1 with $H_{r}^{(\ell)}=H_{1 A, r}^{(\ell)}$. We also let

$$
\chi_{r}^{(\ell)}(g)=\chi_{g}^{X_{A}} \text { for } r=0 \quad(\bmod 2), \quad \chi_{r}^{(\ell)}(g)=\bar{\chi}_{g}^{X_{A}} \text { for } r=1 \quad(\bmod 2) \text {, }
$$


and

$$
\chi^{(\ell)}=\chi_{1 A}^{X_{A}}=\bar{\chi}_{1 A}^{X_{A}}=\frac{24}{\ell-1}
$$

for

$$
X_{A}=X=A_{\ell-1}^{24 /(\ell-1)}
$$

where the characters $\chi_{g}^{X_{A}}$ and $\bar{\chi}_{g}^{X_{A}}$ are defined in the 'Umbral groups' section and given explicitly in 'Euler characters' in Appendix 2. Following the discussion in the 'Shadows' section, we note that the combination (163) of $H_{g, r}^{(\ell)}$ and $H_{r}^{(\ell)}$ has the property of being a modular form rather than a mock modular form, as the shadows of the two contributions to $\widehat{H}_{g, r}^{(\ell)}$ cancel.

Subsequently, we define weight 2 modular forms

$$
F_{g}^{(\ell)}=\sum_{r=1}^{\ell-1} \widehat{H}_{g, r}^{(\ell)} S_{\ell, r}
$$

where $S_{\ell, r}$ is again the unary theta series given in (85). For $\ell>3$, we also specify further weight 2 modular forms by setting

$$
F_{g}^{(\ell), 2}=\sum_{r=1}^{\ell-1} \widehat{H}_{g, r}^{(\ell)} S_{\ell, \ell-r} .
$$

As explained in detail in [1], specifying $F_{g}^{(\ell)}$ and $F_{g}^{(\ell), 2}$ is sufficient to determine $H_{g}^{(\ell)}$ uniquely for $\ell=2,3,5$. In the case $\ell=2$, there is only one term in the sum (167) and it is straightforward to obtain $\widehat{H}_{g, r}^{(\ell)}$ from the weight 2 form $F_{g}^{(\ell)}$. In the case $\ell=3,5$, we also utilise the following fact obeyed by the conjugacy classes of $G^{(\ell)}$.

For any umbral group $G^{(\ell)}$ corresponding to an A-type Niemeier root lattice with Coxeter number $\ell>2$, for a given conjugacy class $[g]$ with $\chi_{1}^{(\ell)}(g)>0$, there exists a (not necessarily different) conjugacy class $\left[g^{\prime}\right]$ with the property

$$
\chi_{1}^{(\ell)}(g)=\chi_{1}^{(\ell)}\left(g^{\prime}\right), \quad \chi_{2}^{(\ell)}(g)=-\chi_{2}^{(\ell)}\left(g^{\prime}\right)
$$

and the order of $g$ and $g^{\prime}$ are either the same or related by a factor of 2 or $1 / 2$. For such paired classes, we have

$$
H_{g, r}^{(\ell)}+(-1)^{r} H_{g^{\prime}, r}^{(\ell)}=0 .
$$

In particular, $H_{g \text {,even }}^{(\ell)}=0$ for the self-paired classes. For $\ell=7,13$, this serves to constrain the function $H_{g}^{(\ell)}$ and supports the claims regarding their modular properties discussed in the 'Modularity' section. We refer the readers to Section 4 of [1] for explicit expressions for the weight 2 forms $F_{g}^{(\ell)}$ and $F_{g}^{(\ell), 2}$.

Note that, from the discussion in the 'Shadows' section, the relation (169) implies that the shadows attached to such paired classes satisfy

$$
S_{g, r}^{(\ell)}+(-1)^{r} S_{g^{\prime}, r}^{(\ell)}=0 .
$$

Therefore, the paired relation (170) can be viewed as a consequence of (the validity of) Conjecture 5 .

\section{Multiplicative relations}

In this subsection, we will describe a web of relations, the multiplicative relations, among the mock modular forms attached to different Niemeier lattices. The nomenclature comes 
from the fact that these relations occur only among mock modular forms attached to Niemeier root systems with one Coxeter number being an integer multiple of the other. To simplify the discussion, we will distinguish the following two types. The horizontal relations relate umbral McKay-Thompson series $H_{g}^{X}$ and $H_{g}^{X^{\prime}}$ attached to different Niemeier root systems with the same Coxeter number, i.e. $m(X)=m\left(X^{\prime}\right)$. The vertical relations connect umbral McKay-Thompson series $H_{g}^{X}$ and $H_{g}^{X^{\prime}}$ attached to different Niemeier root systems $X$ and $X^{\prime}$ with $m(X) \mid m\left(X^{\prime}\right)$ and $m(X) \neq m\left(X^{\prime}\right)$.

First, we will discuss the horizontal relations, summarised in Table 8. Note that there are five pairs of Niemeier root systems that share the same Coxeter number $m \in$ $\{6,10,12,18,30\}$. Let us choose such a pair $\left(X^{\prime}, X\right)$. From the definition of the umbral shadows (118) and the generalisation in the 'Shadows' section to the non-identity conjugacy classes, we see that it can happen that the $r^{\prime}$-th component $S_{g^{\prime}, r^{\prime}}^{X^{\prime}}$ of the shadow attached to the conjugacy class $\left[g^{\prime}\right]$ of the umbral group $G^{X^{\prime}}$ is expressible as a linear combination of the components $S_{g, r}^{X}$ of the shadow attached to the conjugacy class $[g]$ of the umbral group $G^{X}$. It turns out that for all five equal-Coxeter-number pairs of Niemeier root systems, this indeed happens for various pairs $\left(\left[g^{\prime}\right],[g]\right)$ of conjugacy classes.

Note that the relation between the shadows

$$
S_{g^{\prime}, r^{\prime}}^{X^{\prime}}=\sum_{r} c_{r^{\prime}, r} S_{g, r}^{X}
$$

is a necessary but insufficient condition for the linear relation

$$
H_{g^{\prime}, r^{\prime}}^{X^{\prime}}=\sum_{r} c_{r^{\prime}, r} H_{g, r}^{X}
$$

between the corresponding McKay-Thompson series to hold, since the coincidence of the shadow guarantees the coincidence of the corresponding mock modular form only up to the addition of a modular form. Nevertheless, it turns out that in umbral moonshine, the

\begin{tabular}{|c|c|c|c|}
\hline$X^{\prime}$ & $x$ & $\left(\left[g^{\prime}\right],[g]\right)$ & Relations \\
\hline \multirow{7}{*}{$6+3$} & \multirow{7}{*}{6} & $(1 A, 1 A)$ & \multirow{4}{*}{$\begin{array}{l}H_{g^{\prime}, r}^{X^{\prime}}=H_{g, r}^{X}+H_{g, 6-r}^{X} \\
\text { rodd }\end{array}$} \\
\hline & & $(2 A, 2 B)$ & \\
\hline & & $(3 B, 3 A)$ & \\
\hline & & $(4 A, 8 A B)$ & \\
\hline & & $(2 C, 4 A)$ & \multirow{3}{*}{$\begin{array}{l}H_{g^{\prime}, r}^{X^{\prime}}=H_{g, r}^{X}-H_{g, 6-r}^{X} \\
\text { rodd }\end{array}$} \\
\hline & & $(4 B, 8 A B)$ & \\
\hline & & $(6 B, 6 A)$ & \\
\hline \multirow{3}{*}{$10+5$} & \multirow{3}{*}{10} & $(1 A, 1 A)$ & \multirow[t]{2}{*}{$H_{g^{\prime}, r}^{X^{\prime}}=H_{g, r}^{X}+H_{g, 10-r}^{X}$} \\
\hline & & $(2 A, 4 A B)$ & \\
\hline & & $(2 B, 4 A B)$ & $H_{g^{\prime}, r}^{X^{\prime}}=H_{g, r}^{X}-H_{g, 10-r}^{X}$ \\
\hline \multirow{2}{*}{$12+4$} & \multirow{2}{*}{12} & $(1 A, 1 A)$ & $H_{g^{\prime}, r}^{X^{\prime}}=H_{g, r}^{X}+H_{g, 6+r}^{X}, r=1,5$ \\
\hline & & $(2 A, 2 A)$ & $H_{g^{\prime}, 4}^{X^{\prime}}=H_{g, 4}^{X}+H_{g, 8}^{X}$ \\
\hline \multirow{2}{*}{$18+9$} & \multirow{2}{*}{18} & $(1 \mathrm{~A}, 1 \mathrm{~A})$ & $H_{g^{\prime}, r}^{X^{\prime}}=H_{g, r}^{X}+H_{g, 18-r}^{X}$ \\
\hline & & $(2 \mathrm{~A}, 1 \mathrm{~A})$ & $H_{g^{\prime}, r}^{X^{\prime}}=H_{g, r}^{X}-H_{g, 18-r}^{X}$ \\
\hline $30+6,10,15$ & $30+15$ & $(1 \mathrm{~A}, 1 \mathrm{~A})$ & $\begin{array}{l}H_{g^{\prime}, 1}^{X^{\prime}}=H_{g, 1}^{X}+H_{g, 11}^{X} \\
H_{g^{\prime}, 7}^{X^{\prime}}=H_{g, 7}^{X}+H_{g, 13}^{X}\end{array}$ \\
\hline
\end{tabular}

Table 8 Horizontal relations 
relation between the McKay-Thompson series (173) holds whenever the relation between the shadow (172) holds non-trivially with $S_{g^{\prime}, r^{\prime}}^{X^{\prime}}, S_{g, r}^{X} \neq 0$. This fact, together with the more general multiplicative relations (177), can again be viewed as the consequence of the conjectured uniqueness of such mock modular forms (Conjecture 5). See Table 8 for the list of such horizontal relations. In particular, for the identity element, a relation $H_{r^{\prime}}^{X^{\prime}}=\sum_{r} c_{r^{\prime}, r} H_{r}^{X}$ holds for some $c_{r^{\prime}, r} \in \mathbb{Z}$ for all the five pairs $\left(X^{\prime}, X\right)$ of Niemeier root systems with the same Coxeter numbers.

Note that, together with the discussion in the 'From weight 0 Jacobi forms to umbral mock modular forms' section and Proposition 2, this implies more specifically that the umbral mock modular forms $H_{r}^{X^{\prime}}$ can be obtained as the theta coefficients of the finite part of the meromorphic weight 1 Jacobi form

$$
\psi^{X^{\prime}}=-\mu_{1,0} \phi^{X^{\prime}} \mid \mathcal{W}^{X^{\prime}}, \phi^{X^{\prime}}=\phi^{X}
$$

for the four pairs $\left(X^{\prime}, X\right) \in\{(6,6+3),(10,10+5),(12,12+4),(18,18+9)\}$ with A-type root systems $X$ and with the weight 0 Jacobi forms given in Table 7 .

In fact, a linear relation between the shadows attached to different Niemeier root systems can happen more generally and not just among those with the same Coxeter numbers. The first indication that non-trivial relations might exist across different Coxeter numbers is the following property of the building blocks of the umbral shadow. As one can easily check, the unary theta function $S_{m}=\left(S_{m, r}\right)$ defined in (85) at a given index $m$ can be re-expressed in terms of those at a higher index as

$$
S_{m, r}(\tau)=\sum_{\ell=0}^{\left\lceil\frac{n}{2}\right\rceil-1} S_{n m, r+2 m \ell}(n \tau)-\sum_{\ell=1}^{\left\lfloor\frac{n}{2}\right\rfloor} S_{n m, 2 m \ell-r}(n \tau),
$$

for any positive integer $n$. The above equality makes it possible to have the relation

$$
\sum_{r^{\prime}} c_{r, r^{\prime}} S_{g^{\prime}, r^{\prime}}^{X^{\prime}}(k \tau)=S_{g, r}^{X}(\tau)
$$

for some $k \in \mathbb{Z}_{>0}$. We will see that this relation between the umbral shadows does occur for many pairs of Niemeier root systems $\left(X^{\prime}, X\right)$ with $m\left(X^{\prime}\right)=k m(X)$. Moreover, whenever this relation holds non-trivially with $S_{g^{\prime}, r^{\prime}}^{X^{\prime}}, S_{g, r}^{X} \neq 0$, the corresponding relation among the McKay-Thompson series

$$
\sum_{r^{\prime}} c_{r, r^{\prime}} H_{g^{\prime}, r^{\prime}}^{X^{\prime}}\left(\frac{m\left(X^{\prime}\right)}{m(X)} \tau\right)=H_{g, r}^{X}(\tau)
$$

also holds.

We summarise a minimal set of such relations in Table 9. In Tables 8 and 9, when it is not explicitly specified, the relation holds for all values of $r$ such that all $H_{g^{\prime}, r_{1}}^{X^{\prime}}$ and $H_{g, r_{2}}^{X}$ appearing on both sides of the equation have $1 \leq r_{1} \leq m\left(X^{\prime}\right)$ and $1 \leq r_{2} \leq m(X)$. From the relations recorded in these tables as well as the paired relations (213) and (217), many further relations can be derived. For example, combining the relations between the $(1 A, 2 B C)$ classes for $\left(X^{\prime}, X\right)=(16,8)$, the $(2 B C, 4 C)$ classes for $\left(X^{\prime}, X\right)=(8,4)$ and the $(4 C, 8 A)$ classes at $\left(X^{\prime}, X\right)=(4,2)$, one can deduce that there is a multiplicative relation $\sum_{n=0}^{7}(-1)^{n} H_{1 A, 2 n+1}^{(16)}(8 \tau)=H_{8 A, 1}^{(2)}(\tau)$. See the 'Specification' section for more detailed information. 
Table 9 Vertical relations

\begin{tabular}{|c|c|c|c|}
\hline$\overline{X^{\prime}}$ & $x$ & $\left(\left[g^{\prime}\right],[g]\right)$ & Relations \\
\hline \multirow{4}{*}{4} & \multirow{4}{*}{2} & $(1 A, 2 A),(2 B, 4 A)$ & $\left(H_{g^{\prime}, 1}^{X^{\prime}}-H_{g^{\prime}, 3}^{X^{\prime}}\right)(2 \tau)=H_{g, 1}^{X}(\tau)$ \\
\hline & & $(2 C, 4 B),(3 A, 6 A)$ & \\
\hline & & $(4 A, 4 C),(4 C, 8 A)$ & \\
\hline & & $(6 B C, 12 A),(7 A B, 14 A B)$ & \\
\hline \multirow{6}{*}{6} & \multirow{2}{*}{2} & $(1 A, 3 A),(2 B, 6 A)$ & $\sum_{n=0}^{2}(-1)^{n} H_{g^{\prime}, 1+2 n}^{X^{\prime}}(3 \tau)=H_{g, 1}^{X}(\tau)$ \\
\hline & & $(8 A B, 12 A)$ & \\
\hline & \multirow{4}{*}{3} & $(1 A, 2 B),(2 A, 2 C)$ & $\left(H_{g^{\prime}, r}^{X^{\prime}}-H_{g^{\prime}, 6-r}^{X^{\prime}}\right)(2 \tau)=H_{g, r}^{X}(\tau)$ \\
\hline & & $(2 B, 4 C),(4 A, 4 B)$ & \\
\hline & & $(3 A, 6 C),(6 A, 6 D)$ & \\
\hline & & $(8 A B, 8 C D)$ & \\
\hline $6+3$ & 2 & $(5 A, 15 A B)$ & $\sum_{n=0}^{2}(-1)^{n} H_{g^{\prime}, 1+2 n}^{X^{\prime}}(3 \tau)=H_{g, 1}^{X}(\tau)$ \\
\hline \multirow{2}{*}{8} & \multirow{2}{*}{4} & $(1 A, 2 C),(2 B C, 4 C)$ & $\left(H_{g^{\prime}, r}^{X^{\prime}}-H_{g^{\prime}, 8-r}^{X^{\prime}}\right)(2 \tau)=H_{g, r}^{X}(\tau)$ \\
\hline & & $(4 A, 4 B)$ & \\
\hline 9 & 3 & $(1 A, 3 A),(2 B, 6 C)$ & $\left(H_{g^{\prime}, r}^{X^{\prime}}+H_{g^{\prime}, r+6}^{X^{\prime}}-H_{g^{\prime}, 6-r}^{X^{\prime}}\right)(3 \tau)=H_{g, r}^{X}(\tau)$ \\
\hline \multirow{2}{*}{10} & 2 & $(1 A, 5 A),(4 A B, 10 A)$ & $\sum_{n=0}^{4}(-1)^{n} H_{g^{\prime}, 1+2 n}^{X^{\prime}}(5 \tau)=H_{g, 1}^{X}(\tau)$ \\
\hline & 5 & $(1 A, 2 C),(4 A B, 4 C D)$ & $\left(H_{g^{\prime}, r}^{X^{\prime}}-H_{g^{\prime}, 10-r}^{X^{\prime}}\right)(2 \tau)=H_{g, r}^{X}(\tau)$ \\
\hline $10+5$ & 2 & $(3 A, 15 A B)$ & $\left(H_{g^{\prime}, 1}^{X^{\prime}}-H_{g^{\prime}, 3}^{X^{\prime}}+H_{g^{\prime}, 5}^{X^{\prime}} / 2\right)(5 \tau)=H_{g, 1}^{X}(\tau)$ \\
\hline \multirow{2}{*}{12} & 4 & $(1 A, 3 A)$ & $\left(H_{g^{\prime}, r}^{X^{\prime}}+H_{g^{\prime}, r+8}^{X^{\prime}}-H_{g^{\prime}, 8-r}^{X^{\prime}}\right)(3 \tau)=H_{g, r}^{X}(\tau)$ \\
\hline & 6 & $(1 A, 2 B)$ & $\left(H_{g^{\prime}, r}^{X^{\prime}}-H_{g^{\prime}, 12-r}^{X^{\prime}}\right)(2 \tau)=H_{g, r}^{X}(\tau)$ \\
\hline \multirow{2}{*}{$12+4$} & \multirow{2}{*}{6} & $(3 A, 3 A)$ & $\left(H_{g^{\prime}, 1}^{X^{\prime}}-H_{g^{\prime}, 5}^{X^{\prime}}\right)(2 \tau)=\left(H_{g, 1}^{X}-H_{g, 5}^{X}\right)(\tau)$ \\
\hline & & $(2 B, 8 A B)$ & $H_{g, 4}^{X^{\prime}}(2 \tau)=\left(H_{g, 2}^{X}+H_{g, 4}^{X}\right)(\tau)$ \\
\hline \multirow{2}{*}{$14+7$} & \multirow[t]{2}{*}{2} & $(1 A, 7 A B),(2 A, 14 A B)$ & $\left(-\frac{1}{2} H_{g^{\prime}, 7}^{X^{\prime}}+\sum_{n=0}^{2}(-1)^{n} H_{g^{\prime}, 1+2 n}^{X^{\prime}}\right)(7 \tau)=H_{g, 1}^{X}(\tau)$ \\
\hline & & $(3 A, 21 A B)$ & \\
\hline 16 & 8 & $(1 A, 2 B C)$ & $\left(H_{g^{\prime}, r}^{X^{\prime}}-H_{g^{\prime}, 16-r}^{X^{\prime}}\right)(2 \tau)=H_{g, r}^{X}(\tau)$ \\
\hline \multirow[t]{2}{*}{18} & 6 & $(1 A, 3 A)$ & $\left(H_{g^{\prime}, r}^{X^{\prime}}-H_{g^{\prime}, 12-r}^{X^{\prime}}+H_{g^{\prime}, 12+r}^{X^{\prime}}\right)(3 \tau)=H_{g, r}^{X}(\tau)$ \\
\hline & 9 & $(1 A, 2 B)$ & $3\left(H_{g^{\prime}, r}^{X^{\prime}}-H_{g^{\prime}, 18-r}^{X^{\prime}}\right)(2 \tau)=H_{g, r}^{X}(\tau)$ \\
\hline $22+11$ & 2 & $(1 A, 11 A)$ & $\left(-\frac{1}{2} H_{g^{\prime}, 11}^{X^{\prime}}+\sum_{n=0}^{4}(-1)^{n} H_{g^{\prime}, 1+2 n}^{X^{\prime}}\right)(11 \tau)=H_{g, 1}^{X}(\tau)$ \\
\hline 25 & 5 & $(1 A, 5 A)$ & $\left(\sum_{n=0}^{2} H_{g^{\prime}, 10 n+r}^{X^{\prime}}-\sum_{n=1}^{2} H_{g^{\prime}, 10 n-r}^{X^{\prime}}\right)(5 \tau)=H_{g, r}^{X}(\tau)$ \\
\hline \multirow{2}{*}{$30+15$} & 2 & $(1 A, 15 A B)$ & $\left(-\frac{1}{2} H_{g^{\prime}, 15}^{X^{\prime}}+\sum_{n=0}^{6}(-1)^{n} H_{g^{\prime}, 1+2 n}^{X^{\prime}}\right)(15 \tau)=H_{g, 1}^{X}(\tau)$ \\
\hline & $10+5$ & $(1 A, 3 A)$ & $\left(H_{g^{\prime}, r}^{X^{\prime}}+H_{g^{\prime}, 10-r}^{X^{\prime}}-H_{g^{\prime}, 10+r}^{X^{\prime}}\right)(3 \tau)=H_{g, r}^{X}(\tau)$ \\
\hline $46+23$ & 2 & $(1 A, 23 A B)$ & $\left(-\frac{1}{2} H_{g^{\prime}, 23}^{X^{\prime}}+\sum_{n=0}^{10}(-1)^{n} H_{g^{\prime}, 1+2 n}^{X^{\prime}}\right)(23 \tau)=H_{g, 1}^{X}(\tau)$ \\
\hline
\end{tabular}

These multiplicative relations form an intricate web relating umbral moonshine at different lambencies. We summarise this web in Figure 3, where the horizontal relations are indicated by arrowed dashed lines and the vertical relations are indicated by solid lines.

We will finish this section with a discussion of a curious property of the multiplicative relations. Note that these relations occur among the McKay-Thomson series attached to $[g] \subset G^{X}$ and $\left[g^{\prime}\right] \subset G^{X^{\prime}}$ with $\operatorname{ord}\left(g^{\prime}\right) m\left(X^{\prime}\right)$ coinciding with $\operatorname{ord}(g) m(X)$ up to a factor of 2. This property can be understood as a consequence of the relation between the level $n_{g}$ of the automorphy group $\Gamma_{0}\left(n_{g}\right)$ of the McKay-Thomson series $H_{g}^{X}$ and the order $\operatorname{ord}(g)$ of the group element ( $c f$. 'Modularity' section). The extra factor of 2 can be understood as 
a consequence of the structure $G^{X}=2 \cdot \bar{G}^{X}$ of the associated umbral groups ( $c f$. 'Umbral groups' section).

\section{Mock theta functions}

In this subsection, we record relations between the McKay-Thompson series of umbral moonshine and known mock theta functions. Many of the mock theta functions arising appear either in Ramanujan's last letter to Hardy or in his lost notebook [50]. In what follows, we will give explicit expressions for the mock theta series using the $q$-Pochhammer symbol

$$
(a ; q)_{n}=\prod_{k=0}^{n-1}\left(1-a q^{k}\right)
$$

For lambency 2, two of the functions $H_{g}^{(2)}(\tau)$ are related to Ramanujan's mock theta functions of orders 2 and 8 through

$$
\begin{aligned}
& H_{4 B}^{(2)}(\tau)=-2 q^{-1 / 8} \mu(q)=-2 q^{-1 / 8} \sum_{n \geq 0} \frac{(-1)^{n} q^{n^{2}}\left(q ; q^{2}\right)_{n}}{\left(-q^{2} ; q^{2}\right)_{n}^{2}} \\
& H_{8 A}^{(2)}(\tau)=-2 q^{-1 / 8} U_{0}(q)=-2 q^{-1 / 8}=\sum_{n \geq 0} \frac{q^{n^{2}}\left(-q ; q^{2}\right)_{n}}{\left(-q^{4} ; q^{4}\right)_{n}}
\end{aligned}
$$

For $\ell=3$, we encounter the following order 3 mock theta functions of Ramanujan:

$$
\begin{aligned}
& H_{2 B, 1}^{(3)}(\tau)=H_{2 C, 1}^{(3)}(\tau)=H_{4 C, 1}^{(3)}(\tau)=-2 q^{-1 / 12} f\left(q^{2}\right) \\
& H_{6 C, 1}^{(3)}(\tau)=H_{6 D, 1}^{(3)}(\tau)=-2 q^{-1 / 12} \chi\left(q^{2}\right) \\
& H_{8 C, 1}^{(3)}(\tau)=H_{8 D, 1}^{(3)}(\tau)=-2 q^{-1 / 12} \phi\left(-q^{2}\right) \\
& H_{2 B, 2}^{(3)}(\tau)=-H_{2 C, 2}^{(3)}(\tau)=-4 q^{2 / 3} \omega(-q) \\
& H_{6 C, 2}^{(3)}(\tau)=-H_{6 D, 2}^{(3)}(\tau)=2 q^{2 / 3} \rho(-q),
\end{aligned}
$$

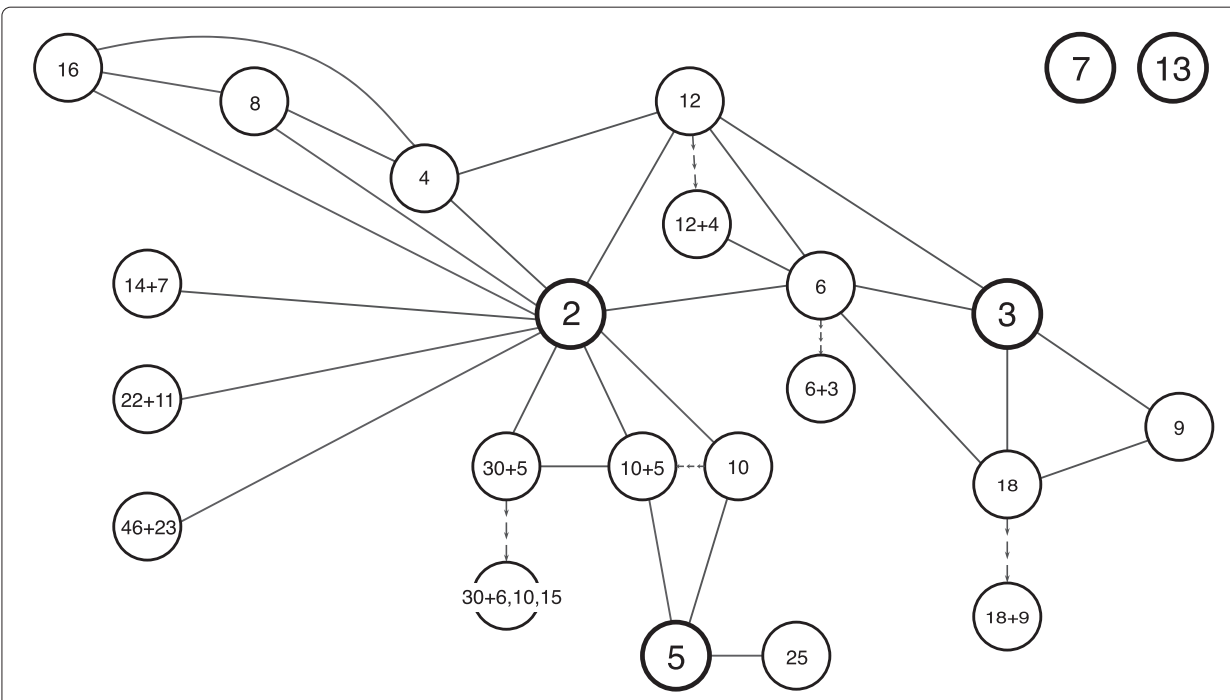

Figure 3 Multiplicative relations. 
where

$$
\begin{aligned}
& f(q)=1+\sum_{n=1}^{\infty} \frac{q^{n^{2}}}{(1+q)^{2}\left(1+q^{2}\right)^{2} \cdots\left(1+q^{n}\right)^{2}} \\
& \phi(q)=1+\sum_{n=1}^{\infty} \frac{q^{n^{2}}}{\left(1+q^{2}\right)\left(1+q^{4}\right) \cdots\left(1+q^{2 n}\right)} \\
& \chi(q)=1+\sum_{n=1}^{\infty} \frac{q^{n^{2}}}{\left(1-q+q^{2}\right)\left(1-q^{2}+q^{4}\right) \cdots\left(1-q^{n}+q^{2 n}\right)} \\
& \omega(q)=\sum_{n=0}^{\infty} \frac{q^{2 n(n+1)}}{(1-q)^{2}\left(1-q^{3}\right)^{2} \cdots\left(1-q^{2 n+1}\right)^{2}} \\
& \rho(q)=\sum_{n=0}^{\infty} \frac{q^{2 n(n+1)}}{\left(1+q+q^{2}\right)\left(1+q^{3}+q^{6}\right) \cdots\left(1+q^{2 n+1}+q^{4 n+2}\right)} .
\end{aligned}
$$

For $\ell=4$ and $\ell=16$, we have the relations

$$
\begin{aligned}
& H_{2 C, 1}^{(4)}(\tau)=q^{-\frac{1}{16}}\left(-2 S_{0}(q)+4 T_{0}(q)\right), \\
& H_{2 C, 3}^{(4)}(\tau)=q^{\frac{7}{16}}\left(2 S_{1}(q)-4 T_{1}(q)\right), \\
& H_{4 C, 1}^{(4)}(\tau)=-2 q^{-\frac{1}{16}} S_{0}(q), \\
& H_{4 C, 3}^{(4)}(\tau)=2 q^{\frac{7}{16}} S_{1}(q)
\end{aligned}
$$

and

$$
\begin{aligned}
& H_{1 A, 2}^{(16)}(\tau)=H_{1 A, 14}^{(16)}=2 q^{-1 / 16} T_{0}(-q)=\frac{1}{2}\left(H_{2 C, 1}^{(4)}(\tau)-H_{4 C, 1}^{(4)}(\tau)\right) \\
& H_{1 A, 4}^{(16)}(\tau)=H_{1 A, 12}^{(16)}=2 q^{-1 / 4} V_{1}(q) \\
& H_{1 A, 6}^{(16)}(\tau)=H_{1 A, 10}^{(16)}=2 q^{7 / 16} T_{1}(-q)
\end{aligned}
$$

to the order 8 mock theta functions

$$
\begin{aligned}
& S_{0}(\tau)=\sum_{n \geq 0} \frac{q^{n^{2}}\left(-q ; q^{2}\right)_{n}}{\left(-q^{2} ; q^{2}\right)_{n}} \\
& S_{1}(\tau)=\sum_{n \geq 0} \frac{q^{n(n+2)}\left(-q ; q^{2}\right)_{n}}{\left(-q^{2} ; q^{2}\right)_{n}} \\
& T_{0}(\tau)=\sum_{n \geq 0} \frac{q^{(n+1)(n+2)}\left(-q^{2} ; q^{2}\right)_{n}}{\left(-q ; q^{2}\right)_{n+1}} \\
& T_{1}(\tau)=\sum_{n \geq 0} \frac{q^{n(n+1)}\left(-q^{2} ; q^{2}\right)_{n}}{\left(-q ; q^{2}\right)_{n+1}} \\
& V_{1}(\tau)=\sum_{n \geq 0} \frac{q^{(n+1)^{2}}\left(-q ; q^{2}\right)_{n}}{\left(q ; q^{2}\right)_{n+1}}
\end{aligned}
$$

discussed in [51]. We also have $H_{2 A, r}^{(16)}=-H_{1 A, r}^{(16)}$ for $r=2,4,6,10,12,14$.

For $\ell=5$, we encounter four of Ramanujan's order 10 mock theta functions:

$$
\begin{aligned}
& H_{2 B C, 1}^{(5)}(\tau)=H_{4 C D, 1}^{(5)}(\tau)=-2 q^{-\frac{1}{20}} X\left(q^{2}\right) \\
& H_{2 B C, 3}^{(5)}(\tau)=H_{4 C D, 3}^{(5)}(\tau)=-2 q^{-\frac{9}{20}} \chi_{10}\left(q^{2}\right) \\
& H_{2 C, 2}^{(5)}(\tau)=-H_{2 B, 2}^{(5)}(\tau)=2 q^{-\frac{1}{5}} \psi_{10}(-q) \\
& H_{2 C, 4}^{(5)}(\tau)=-H_{2 B, 4}^{(5)}(\tau)=-2 q^{\frac{1}{5}} \phi_{10}(-q)
\end{aligned}
$$


given by

$$
\begin{aligned}
\phi_{10}(q) & =\sum_{n=0}^{\infty} \frac{q^{n(n+1) / 2}}{\left(q ; q^{2}\right)_{n+1}} \\
\psi_{10}(q) & =\sum_{n=0}^{\infty} \frac{q^{(n+1)(n+2) / 2}}{\left(q ; q^{2}\right)_{n+1}} \\
X(q) & =\sum_{n=0}^{\infty} \frac{(-1)^{n} q^{n^{2}}}{(-q ; q)_{2 n}} \\
\chi_{10}(q) & =\sum_{n=0}^{\infty} \frac{(-1)^{n} q^{(n+1)^{2}}}{(-q ; q)_{2 n+1}} .
\end{aligned}
$$

At $\ell=6$ and $\ell=6+3$, we find

$$
\begin{aligned}
H_{2 B, 3}^{(6)}(\tau) & =-2 q^{-3 / 8} \psi_{6}(q) \\
H_{2 A, 1}^{(6+3)}(\tau) & =-2 q^{-1 / 24} \phi_{6}(q) \\
H_{3 B, 1}^{(6+3)}(\tau) & =-2 q^{-1 / 24} \gamma_{6}(q) \\
H_{2 B, 1}^{(6+3)}(\tau) & =-2 q^{-1 / 24} f(q) \\
H_{4 B, 1}^{(6+3)}(\tau) & =-2 q^{-1 / 24} \phi(-q) \\
H_{6 B, 1}^{(6+3)}(\tau) & =-2 q^{-1 / 24} \chi(q)
\end{aligned}
$$

where $f(q), \phi(q), \chi(q)$ are third-order mock theta functions given earlier and

$$
\begin{aligned}
& \psi_{6}(q)=\sum_{n=0}^{\infty} \frac{(-1)^{n} q^{(n+1)^{2}}\left(q ; q^{2}\right)_{n}}{(-q ; q)_{2 n+1}} \\
& \phi_{6}(q)=\sum_{n=0}^{\infty} \frac{(-1)^{n} q^{n^{2}}\left(q ; q^{2}\right)_{n}}{(-q ; q)_{2 n}} \\
& \gamma_{6}(q)=\sum_{n=0}^{\infty} \frac{q^{n^{2}}(q ; q)_{n}}{\left(q^{3} ; q^{3}\right)_{n}}
\end{aligned}
$$

are sixth-order mock theta functions.

$$
\text { For } \ell=8 \text {, }
$$

$$
\begin{aligned}
& H_{1 A, 2}^{(8)}(\tau)=H_{1 A, 6}^{(8)}=4 q^{-1 / 4} A(q)=4 q^{-1 / 4} \sum_{n \geq 0} \frac{q^{n+1}\left(-q^{2} ; q^{2}\right)_{n}}{\left(q ; q^{2}\right)_{n+1}} \\
& H_{1 A, 4}^{(8)}(\tau)=4 q^{1 / 2} B(q)=4 q^{1 / 2} \sum_{n \geq 0} \frac{q^{n}\left(-q ; q^{2}\right)_{n}}{\left(q ; q^{2}\right)_{n+1}}
\end{aligned}
$$

where $A(q), B(q)$ are both second-order mock theta functions discussed in [52]. 
For $\ell=12$ and $\ell=12+4$, we have

$$
\begin{gathered}
H_{1 A, 2}^{(12)}(\tau)=H_{1 A, 10}^{(12)}(\tau)=-2 q^{-4 / 48} \sigma(q) \\
H_{1 A, 4}^{(12)}(\tau)=H_{1 A, 8}^{(12)}(\tau)=2 q^{2 / 3} \omega(q) \\
H_{1 A, 1}^{(12+4)}(\tau)=-q^{-1 / 48}\left(f\left(q^{1 / 2}\right)-f\left(-q^{1 / 2}\right)\right)
\end{gathered}
$$

where $\sigma(q)$ is the order 6 mock theta function

$$
\sigma(q)=\sum_{n=0}^{\infty} \frac{q^{(n+1)(n+2) / 2}(-q ; q)_{n}}{\left(q ; q^{2}\right)_{n+1}}
$$

and $\omega(q)$ and $f(q)$ are third-order mock theta functions given in (181).

For $\ell=30+6,10,15$, we find four of Ramanujan's mock theta functions of order 5 :

$$
\begin{aligned}
& H_{1 A, 1}^{(30+6,10,15)}(\tau)=q^{-1 / 120}\left(2 \chi_{0}(q)-4\right) \\
& H_{1 A, 7}^{(30+6,10,15)}(\tau)=2 q^{71 / 120} \chi_{1}(q) \\
& H_{2 A, 1}^{(30+6,10,15)}(\tau)=-2 q^{-1 / 120} \phi_{0}(-q) \\
& H_{2 A, 7}^{(30+6,10,15)}(\tau)=2 q^{-49 / 120} \phi_{1}(-q)
\end{aligned}
$$

where

$$
\begin{aligned}
\chi_{0}(\tau) & =\sum_{n \geq 0} \frac{q^{n}}{\left(q^{n+1} ; q\right)_{n}} \\
\chi_{1}(\tau) & =\sum_{n \geq 0} \frac{q^{n}}{\left(q^{n+1} ; q\right)_{n+1}} \\
\phi_{0}(\tau) & =\sum_{n \geq 0} q^{n^{2}}\left(-q ; q^{2}\right)_{n} \\
\phi_{1}(\tau) & =\sum_{n \geq 0} q^{(n+1)^{2}}\left(-q ; q^{2}\right)_{n} .
\end{aligned}
$$

\section{Specification}

In this subsection, we will combine the different types of data on the McKay-Thompson series discussed in the 'From weight 0 Jacobi forms to umbral mock modular forms' section and the 'Prime lambencies' to 'Mock theta functions' sections, and explain how they lead to explicit expressions for the umbral McKay-Thompson series.

First, we will note one more relation among umbral McKay-Thompson series $H_{g}^{X}$ and $H_{g^{\prime}}^{X}$ attached to different conjugacy classes of the same umbral group $G^{X}$. Notice that for all the A-type Niemeier root systems as well as $X=E_{6}^{4}$, the corresponding umbral group takes the form

$$
G^{X}=2 \cdot \bar{G}^{X}
$$

and the corresponding McKay-Thompson series display the following paired relation. For the A-type Niemeier root system $X$, for every conjugacy class $[g] \subset G^{X}$ with $\bar{\chi}_{g}^{X_{A}}>0$, there is a unique conjugacy class $\left[g^{\prime}\right]$ with

$$
\chi_{g}^{X_{A}}=-\chi_{g^{\prime}}^{X_{A}}, \quad \bar{\chi}_{g}^{X_{A}}=\bar{\chi}_{g^{\prime}}^{X_{A}},
$$

that we say to be paired with $[g]$. For such paired classes, the corresponding McKayThompson series satisfy the relation

$$
H_{g, r}^{X}+(-1)^{r} H_{g^{\prime}, r}^{X}=0
$$


This generalises the paired property for the pure A-series discussed in the 'Prime lambencies' section. Similarly, for $X=E_{6}^{4}$, we have for every conjugacy class $[g] \subset G^{X}$ with $\chi_{g}^{X_{E}}>0$ a conjugacy class $\left[g^{\prime}\right]$ with

$$
\chi_{g}^{X_{E}}=-\chi_{g^{\prime}}^{X_{E}}, \quad \bar{\chi}_{g}^{X_{E}}=\bar{\chi}_{g^{\prime}}^{X_{E}},
$$

that is paired with $[g]$. For such paired classes, the corresponding McKay-Thompson series again satisfy the relation (213).

For the lambency $\ell=6+3$, corresponding to $X=D_{4}^{6}$, the corresponding umbral group has the form

$$
G^{X}=3 . \bar{G}^{X}
$$

and the conjugacy classes with $\bar{\chi}^{X_{D}}(g)>0$ form pairs satisfying

$$
\chi_{g}^{X_{D}}=\chi_{g^{\prime}}^{X_{D}}, \quad \check{\chi}_{g}^{X_{D}}+2 \check{\chi}_{g^{\prime}}^{X_{D}}=0 .
$$

For these paired conjugacy classes, the McKay-Thompson series have the property

$$
H_{g, 1}^{X}-H_{g^{\prime}, 1}^{X}=0, \quad H_{g, 3}^{X}+2 H_{g^{\prime}, 3}^{X}=0 .
$$

In particular, we have $H_{g, 3}^{(6+3)}=0$ for all the self-paired classes. From the discussion in the 'Shadows' section, we see that the relation between the twisted Euler characters and the paired relations is implied by the shadows of the umbral McKay-Thompson series, just as the multiplicative relations discussed in the 'Multiplicative relations' section. As a result, one can view the relations (213) and (217) as a consequence of the apparently general phenomenon that the umbral McKay-Thompson series are determined by their mock modular properties together with the optimal growth condition, $c f$. Conjecture 5 .

In the rest of this subsection, we will tie these relations together and discuss each lambency separately. First of all, for all the A-type Niemeier root systems

$$
\ell \in\{2,3,4,5,6,7,8,9,10,12,13,16,18,25\},
$$

the discussion in the 'From weight 0 Jacobi forms to umbral mock modular forms' section specifies all $H^{(\ell)}=\left(H_{1 A, r}^{(\ell)}\right), r=1, \ldots, \ell-1$ and it remains only to specify $H_{g}^{(\ell)}$ with $[g] \subset G^{X}$ different from the identity class. For $\ell=2,3,5$, the discussion in the 'Prime lambencies' section specifies all the McKay-Thompson series. For $\ell=7,13$, the discussion in the 'Prime lambencies' gives partial information on all the McKay-Thompson series. For $\ell=4$, the vertical relations in Table 9 specify all the odd components of $H_{g, r}^{(4)}$ for all conjugacy classes $[g] \in G^{(4)}$ except for $[g]=4 B, 8 A$. The odd components for $[g]=4 B, 8 A$ can be specified by the identities

$$
\begin{aligned}
& \left(H_{4 B, 1}^{(4)}-H_{4 B, 3}^{(4)}\right)(\tau)=-2 \frac{\eta(\tau / 2) \eta(\tau)^{4}}{\eta(\tau)^{2} \eta(4 \tau)^{2}}, \\
& \left(H_{8 A, 1}^{(4)}-H_{8 A, 3}^{(4)}\right)(\tau)=-2 \frac{\eta(\tau)^{3}}{\eta(\tau / 2) \eta(4 \tau)} .
\end{aligned}
$$

We are left to determine the second components $H_{g, 2}^{(4)}(\tau)$, which are given by

$$
\begin{aligned}
H_{3 A, 2}^{(4)}(\tau)= & \frac{1}{4} H_{2}^{(4)}(\tau)+\frac{1}{2 \eta(2 \tau)^{3}}\left(-3 \Lambda_{2}(\tau)-4 \Lambda_{3}(\tau)+\Lambda_{6}(\tau)\right), \\
H_{7 A B, 2}^{(4)}(\tau)= & \frac{1}{8} H_{2}^{(4)}(\tau) \\
& +\frac{1}{12 \eta(2 \tau)^{3}}\left(-7 \Lambda_{2}(\tau)-4 \Lambda_{7}(\tau)+\Lambda_{14}(\tau)+28 f_{14}(\tau)\right) .
\end{aligned}
$$


Together with the paired relation (213), Table 9 and

$$
H_{g, 2}^{(4)}(\tau)=0 \quad \text { for all }[g] \notin\{1 A, 2 A, 3 A, 6 A, 7 A B, 14 A B\},
$$

(219)-(220) completely specifies all $H_{g}^{(4)}=\left(H_{g, r}^{(4)}\right)$ for all $[g] \subset G^{(4)}$.

For $\ell=6$, the vertical relations in Table 9 to the McKay-Thompson series of lambencies $\ell=2,3$ suffice to specify all $H_{g}^{(6)}=\left(H_{g, r}^{(6)}\right)$ except for the components $r=1,5$ of the classes $3 A$ and $6 A$. Subsequently, the relation to $H_{1 A, r}^{(18)}$ in Table 9 and the paired relation (213) determines $H_{3 A}^{(18)}$ and $H_{6 A}^{(18)}$.

For $\ell=8$, the vertical relations to $\ell=4$ recorded in Table 9, together with the paired relation (213) and $H_{2 B C, 2 n}^{(8)}=H_{4 A, 2 n}^{(8)}=0$ for all $n=1,2,3$, specify all the $H_{g}^{(8)}=\left(H_{g, r}^{(8)}\right)$.

For $\ell=9$, the vertical relations to $\ell=3$ recorded in Table 9 together with the paired relation (213) determine all $H_{g}^{(9)}$ except for $[g]=3 A, 6 A$. To determine $H_{3 A}^{(9)}$ and $H_{6 A}^{(9)}$, we have

$$
\begin{aligned}
\left(H_{3 A, 1}^{(9)}-H_{3 A, 5}^{(9)}+H_{3 A, 7}^{(9)}\right)(3 \tau)= & -6 \frac{\eta(\tau) \eta(12 \tau) \eta(18 \tau)^{2}}{\eta(6 \tau) \eta(9 \tau) \eta(36 \tau)} \\
\left(H_{3 A, 2}^{(9)}-H_{3 A, 4}^{(9)}+H_{3 A, 8}^{(9)}\right)(3 \tau)= & -3 \frac{\eta(\tau) \eta(2 \tau) \eta(3 \tau)^{2}}{\eta(4 \tau)^{2} \eta(9 \tau)} \\
& +3 \frac{\eta(2 \tau)^{6} \eta(12 \tau) \eta(18 \tau)^{2}}{\eta(\tau) \eta(4 \tau)^{4} \eta(6 \tau) \eta(9 \tau) \eta(36 \tau)} \\
H_{3 A, 3}^{(9)}(\tau)=H_{3 A, 6}^{(9)}(\tau)= & H_{6 A, 3}^{(9)}(\tau)=H_{6 A, 6}^{(9)}(\tau)=0 .
\end{aligned}
$$

Together with the paired relation, this determines all $H_{3 A, r}^{(9)}$ and $H_{6 A, r}^{(9)}$ and finishes the specification for $\ell=9$.

For $\ell=10$, the vertical relations to $\ell=2$ and $\ell=5$ recorded in Table 9 specify all $H_{g}^{(10)}$. For $\ell=12,16,18,25$, there is only one conjugacy class $2 A$ except for the identity class. The paired relation (213) relating the McKay-Thompson series for the $2 A$ class to that of the identity class then determines all $H_{g}^{(\ell)}$.

Next, we turn to the D-type Niemeier root systems. For $\ell=6+3$, the horizontal relations in Table 8 determine all McKay-Thompson series $H_{g}^{(6+3)}$, except for $[g] \in\{3 C, 6 C\}$ that are given by

$$
\begin{aligned}
H_{3 C, 1}^{(6+3)}(\tau) & =-2 \frac{\eta^{2}(\tau)}{\eta(3 \tau)}, H_{6 C, 1}^{(6+3)}(\tau)=-2 \frac{\eta(2 \tau) \eta(3 \tau)}{\eta(6 \tau)}, \\
H_{3 C, 3}^{(6+3)} & =H_{6 C, 3}^{(6+3)}=0 .
\end{aligned}
$$

For $\ell=10+5$, the horizontal relations in Table 8 and the vertical relations in Table 9 determine all McKay-Thompson series $H_{g}^{(10+5)}$, except for $[g]=4 A$. For $\ell=14+7$, the vertical relations in Table 9 determine all McKay-Thompson series $H_{g}^{(14+7)}$. For $\ell=$ $18+9$, the horizontal relations in Table 8 determine all McKay-Thompson series $H_{g}^{(18+9)}$. For $\ell=22+11$, the vertical relations in Table 9 determine the umbral form $H_{1 A}^{(22+11)}$. For $\ell=30+15$ and $\ell=46+23$, the vertical relations in Table 9 determine the only McKay-Thompson series $H_{1 A}^{(\ell)}$ attached to the corresponding Niemeier root system.

Finally, we discuss the McKay-Thompson series attached to the two E-type Niemeier root systems. For $\ell=12+4$, the horizontal relations in Table 8 and the vertical relations in Table 9 determine all the McKay-Thompson series $H_{g}^{(12+4)}$ except for $[g] \in\{6 A, 8 A B\}$. For $\ell=30+6,10,15$, the horizontal relations in Tables 8 and 9 determine the umbral mock modular form $H_{1 A}^{(30+6,10,15)}$. 
Focusing on the umbral mock modular form $H^{X}=H_{1 A}^{X}$ corresponding to the identity class, one can explicitly check that the above specification determines the unique vectorvalued mock modular form satisfying the conditions of Corollary 2 .

Proposition 6. Let $X$ be one of the D-or E-type Niemeier root systems (cf. (12), (13)). The vector-valued mock modular form $H^{X}=H_{1 A}^{X}$ specified above is the unique vectorvalued mock modular form with shadow $S^{X}$ satisfying the optimal growth condition (cf. Corollary 2).

Together with Proposition 5, this proposition establishes our construction of the unique vector-valued mock modular form $H^{X}$ corresponding to all 23 Niemeier root systems $X$.

\section{Conjectures}

In this section, we pose the umbral moonshine conjectures connecting the umbral groups $G^{X}$ and the mock modular forms $H_{g}^{X}$ discussed in the previous sections.

\section{Modules}

In this section, we formulate a conjecture that relates the umbral McKay-Thompson series $H_{g}^{X}$ to an infinite-dimensional $G^{X}$-module $K^{X}$.

Recall that a super-space $V$ is a $\mathbb{Z} / 2 \mathbb{Z}$-graded vector space $V=V_{\overline{0}} \oplus V_{\overline{1}}$. If $T: V \rightarrow V$ is a linear operator preserving the grading, then the super-trace of $T$ is given by $\operatorname{str}_{V} T=$ $\operatorname{tr}_{V_{\overline{0}}} T-\operatorname{tr}_{V_{\overline{1}}} T$ where $\operatorname{tr}_{W} T$ denotes the usual trace of $T$ on $W$. We say that $V$ is purely even (odd) when $V=V_{\overline{0}}\left(V_{\overline{1}}\right)$. If $V$ is a $G$-module, with $G$-action preserving the $\mathbb{Z} / 2 \mathbb{Z}$ grading, then the function $g \mapsto \operatorname{str}_{V} g$ is called the super-character of $G$ determined by $V$.

Conjecture 1. Let $X$ be a Niemeier root system and let $m$ be the Coxeter number of $X$.

There exists a naturally defined $\mathbb{Z} / 2 m \mathbb{Z} \times \mathbb{Q}$-graded super-module

$$
K^{X}=\bigoplus_{r(\bmod 2 m)} K_{r}^{X}=\bigoplus_{r(\bmod 2 m)} \bigoplus_{\substack{D \in \mathbb{Z} \\ D=r^{2}(\bmod 4 m)}} K_{r,-D / 4 m}^{X}
$$

for $G^{X}$ such that the graded super-character attached to an element $g \in G^{X}$ coincides with the vector-valued mock modular form

$$
c^{X} H_{g, r}^{X}(\tau)=\sum_{\substack{D \in \mathbb{Z} \\ D=r^{2}(\bmod 2 m)}} \operatorname{str}_{K_{r,-D / 4 m}^{X}}(g) q^{-D / 4 m},
$$

where $c^{X}=1$ except for $X=A_{8}^{3}$, for which $c^{X}=3$. Moreover, the homogeneous component $K_{r, d}^{X}$ of $K^{X}$ is purely even if $d>0$.

The reason for the exceptional value $c^{X}=3$ for $X=A_{8}^{3}$ is the curious fact that there are no integer combinations of irreducible characters of $G^{X}$ that coincide with the coefficients $q^{-D / 36}, D=-27 \lambda^{2}$ for some integer $\lambda$, of $H_{g, r}^{X}(\tau)$ (cf. Conjecture 8). For example, the minimal positive integer $c$ for which $g \mapsto c H_{g, 6}^{X}$ is a graded virtual super-character of $G^{X}$ is $c=c^{X}=3$.

Combining the above conjecture and the paired relations (213) and (217) of the McKayThompson series, we arrive at the following. 
Conjecture 2. Let $X$ be a Niemeier root system and set $c=\# G^{X} / \# \bar{G}^{X}$. Then, the $\mathbb{Q}$-graded $G^{X}$-module $K_{r}^{X}$ is a faithful representation of $G^{X}$ when $r=0(\bmod c)$ and factors through $\bar{G}^{X}$ otherwise.

As discussed in the 'Groups' section, we have $c=2$ for $\ell \in\{3,4,5,6,7,8,9,10$, $12,13,16,18,25,12+4\}$ and $c=3$ for $\ell=6+3$, and $c=1$ in the remaining 8 cases.

As mentioned in the 'Background' section, for the special case $X=A_{1}^{24}$, Conjecture 1 has been shown to be true by T. Gannon in [16], although the construction of $K^{X}$ is still absent. In this case, we have $c=1$ and hence Conjecture 2 is automatically true. It should be possible to apply the techniques similar to that in [16] to prove Conjecture 1 for other Niemeier root systems $X$.

\section{Modularity}

We have attached a cusp form $S_{g}^{X}$ to each $g \in G^{X}$ in the 'Shadows' section by utilising the naturally defined permutation representations of $G^{X}$, and the corresponding twisted Euler characters, that are described in the 'Umbral groups' section. We begin this section with an explicit formulation of the conjecture that these cusp forms describe the shadows of the super-characters attached to the conjectural $G^{X}$-module $K^{X}$.

In preparation for the statement, define $n_{g}$ and $h_{g}$ for $g \in G^{X}$ as follows. Take $n_{g}$ to be the order of the image of $g$ in $\bar{G}^{X}$ ( $c f$. 'Umbral groups' section), and set $h_{g}=N_{g} / n_{g}$ where $N_{g}$ denotes the product of the shortest and longest cycle lengths of the permutation which is the image of $g$ under $G^{X} \rightarrow \operatorname{Sym}_{\Phi}$. These values are on display in the tables of 'Euler characters' in Appendix 2. They may also be read off from the cycle shapes $\widetilde{\Pi}_{g}^{X}$ and $\bar{\Pi}_{g}^{X_{A}}, \bar{\Pi}_{g}^{X_{D}}$, \&c., attached to the permutation representations constructed in the 'Umbral groups' section, for $n_{g}$ is the maximum of the cycle lengths appearing in $\bar{\Pi}_{g}^{X_{A}}, \bar{\Pi}_{g}^{X_{D}}$ and $\bar{\Pi}_{g}^{X_{E}}$, and if $\widetilde{\Pi}_{g}^{X}=j_{1}^{m_{1}} \cdots j_{k}^{m_{k}}$ with $j_{1}^{m_{1}}<\cdots<j_{1}^{m_{k}}$ and $m_{i}>0$, then $h_{g}=j_{1} j_{k} / n_{g}$.

Conjecture 3. The graded super-characters (224) for fixed $X$ and $g \in G^{X}$ and varying $r \in \mathbb{Z} / 2 m \mathbb{Z}$ define the components of a vector-valued mock modular form $H_{g}^{X}$ of weight $1 / 2$ on $\Gamma_{0}\left(n_{g}\right)$ with shadow function $S_{g}^{X}$.

Let $v_{g}^{X}$ denote the multiplier system of $H_{g}^{X}$. Since the multiplier system of a mock modular form is the inverse of the multiplier system of its shadow, Conjecture 3 completely determines the modular properties of $H_{g}^{X}$ - i.e. the matrix-valued function $v_{g}^{X}$ - when $S_{g}^{X}$ is non-vanishing. However, it may happen that $S_{g}^{X}$ vanishes identically and $H_{g}^{X}$ is a (an honest) modular form. The following conjecture puts a strong restriction on $v_{g}^{X}$ even in the case of vanishing shadow.

Conjecture 4. The multiplier system $v_{g}^{X}$ for $H_{g}^{X}$ coincides with the inverse of the multiplier system for $S^{X}$ when restricted to $\Gamma_{0}\left(n_{g} h_{g}\right)$.

\section{Moonshine}

We now formulate a conjecture which may be regarded as the analogue of the principal modulus property (often referred to as the genus zero property) of monstrous moonshine.

The monstrous McKay-Thompson series $T_{g}$, for $g$ an element of the monster, are distinguished in that each one is a principal modulus with pole at infinity for a genus zero group 
$\Gamma_{g}$, meaning that $T_{g}$ is a $\Gamma_{g}$-invariant holomorphic function on the upper-half plane having a simple pole at the infinite cusp of $\Gamma_{g}$, but having no poles at any other cusps of $\Gamma_{g}$. Equivalently, $T_{g}$ satisfies the conditions

(i) $\left.T_{g}\right|_{1,0} \gamma=T_{g}$ for all $\gamma \in \Gamma_{g}$,

(ii) $q T_{g}(\tau)=O(1)$ as $\tau \rightarrow i \infty$,

(iii) $T_{g}(\tau)=O(1)$ as $\tau \rightarrow \alpha \in \mathbb{Q}$ whenever $\infty \notin \Gamma_{g} \alpha$,

for some group $\Gamma_{g}$, where $\left.T_{g}\right|_{1,0} \gamma$ is the function $\tau \mapsto T_{g}(\gamma \tau)$ by definition (cf. (51)). Note that the existence of a non-constant function $T_{g}$ satisfying the conditions (225) implies that the group $\Gamma_{g}$ has genus zero, for such a function necessarily induces an isomorphism from $X_{\Gamma_{g}}\left(c f\right.$. (17)) to the Riemann sphere. As such, if we assume that both $T_{g}$ and $T_{g}^{\prime}$ satisfy these conditions and both $q T_{g}$ and $q T_{g}^{\prime}$ have the expansion $1+O(q)$ near $\tau \rightarrow i \infty$, then $T_{g}$ and $T_{g}^{\prime}$ differ by an additive constant, i.e. $T_{g}^{\prime}=T_{g}+C$ for some $C \in \mathbb{C}$. In other words, the space of solutions to (225) is 1 or 2 dimensional, according as the genus of $X_{\Gamma_{g}}$ is positive or 0 .

Observe the similarity between condition (ii) of (225) and the optimal growth condition (121). Since $q^{-1}$ is the minimal polar term possible for a non-constant $\Gamma_{g}$-invariant function on the upper-half plane, assuming that the stabiliser of infinity in $\Gamma_{g}$ is generated by $\pm\left(\begin{array}{ll}1 & 1 \\ 0 & 1\end{array}\right)$, the condition (ii) is an optimal growth condition on modular forms of weight 0 ; the coefficients of a form with higher order poles will grow more quickly. The condition (iii) naturally extends this to the situation that $\Gamma_{g}$ has more than one cusp.

Accordingly, we now formulate an analogue of (225) - and an extension of the optimal growth condition (121) to vector-valued mock modular forms of higher level - as follows. Suppose that $v$ is a (matrix-valued) multiplier system on $\Gamma_{0}(n)$ with weight $1 / 2$, and suppose, for the sake of concreteness, that $v$ coincides with the inverse of the multiplier system of $S^{X}$, for some Niemeier root system $X$, when restricted to $\Gamma_{0}(N)$ for some $N$ with $n \mid N$. Observe that, under these hypotheses, every component $H_{r}$ has a Fourier expansion in powers of $q^{1 / 4 m}$ where $m$ is the Coxeter number of $X$, so $q^{-1 / 4 m}$ is the smallest order pole that any component of $H$ may have. Say that a vector-valued function $H=\left(H_{r}\right)$ is a mock modular form of optimal growth for $\Gamma_{0}(n)$ with multiplier $v$, weight $1 / 2$ and shadow $S$ if

(i) $\left.H\right|_{1 / 2, v, S \gamma}=H$ for all $\gamma \in \Gamma_{0}(n)$,

(ii) $q^{1 / 4 m} H_{r}(\tau)=O(1)$ as $\tau \rightarrow i \infty$ for all $r$,

(iii) $H_{r}(\tau)=O(1)$ for all $r$ as $\tau \rightarrow \alpha \in \mathbb{Q}$, whenever $\infty \notin \Gamma_{g} \alpha$.

In condition (i) of (226) we write $\left.\right|_{v, 1 / 2, S}$ for the weight $1 / 2$ action of $\Gamma_{0}(n)$ with multiplier $v$ and twist by $S(c f .(52))$, on holomorphic vector-valued functions on the upper-half plane.

Recall that $v_{g}^{X}$ denotes the multiplier system of $H_{g}^{X}$, and $S_{g}^{X}$ is its shadow. Recall also that $n_{g}$ denotes the order of (the image of) $g \in G^{X}$ in the quotient group $\bar{G}^{X}$. We now conjecture that the umbral McKay-Thompson series all have optimal growth in the sense of (226), and this serves as a direct analogue of the Conway-Norton conjecture of monstrous moonshine, that all the monstrous McKay-Thompson series are principal moduli for genus zero subgroups of $S L_{2}(\mathbb{R})$; or equivalently, that they are all functions of optimal growth in the sense of (225). 
Conjecture 5. Let $X$ be a Niemeier root system and let $g \in G^{X}$. Then, $H_{g}^{X}$ is the unique, up to scale, mock modular form of optimal growth for $\Gamma_{0}\left(n_{g}\right)$ with multiplier $v_{g}^{X}$, weight $1 / 2$ and shadow $S_{g}^{X}$.

Conjecture 5 should serve as an important step in obtaining a characterisation of the mock modular forms of umbral moonshine. Note that the above conjecture has been proven in the 'The umbral shadows' section ( $c f$. Corollary 2 ) for the identity class of $G^{X}$ for all Niemeier root systems $X$. Note that in the case of the identity class, we have $\Gamma_{0}(n)=$ $S L_{2}(\mathbb{Z})$ which has the cusp (representative) at $i \infty$ as the only cusp. As a result, the more general conditions in (226) reduce to the condition (121) discussed in the 'The umbral mock modular forms' section. For $X=A_{1}^{24}$, this conjecture was proven for all conjugacy classes of $G^{X}=M_{24}$ in [53]. See also [54] for related results in this case.

\section{Discriminants}

One of the most striking features of umbral moonshine is the apparently intimate relation between the number fields on which the irreducible representations of $G^{X}$ are defined and the discriminants of the vector-valued mock modular form $H^{X}$. In this subsection, we will discuss this 'discriminant property', extending the discussion in [1].

First, for a Niemeier root system with Coxeter number $m$, we observe that the discriminants of the components $H_{r}^{X}$ of the mock modular form $H^{X}=H_{1 A}^{X}$ determine some important properties of the representations of $G^{X}$. Here, we say that integer $D$ is a discriminant of $H^{X}$ if there exists a term $q^{d}=q^{-\frac{D}{4 m}}$ with non-vanishing Fourier coefficient in at least one of the components. The following result can be verified explicitly using the tables in Appendix 2 and 2.

Proposition 7. Let $X$ be one of the 23 Niemeier root systems. If $n>1$ is an integer satisfying

1. there exists an element of $G^{X}$ of order $n$, and

2. there exists an integer $\lambda$ that satisfies at least one of the following conditions and such that $D=-n \lambda^{2}$ is a discriminant of $H^{X}$. First, $(n, \lambda)=1$, and second, $\lambda^{2}$ is a proper divisor of $n$,

then there exists at least one pair of irreducible representations $\varrho$ and $\varrho^{*}$ of $G^{X}$ and at least one element $g \in G^{X}$ such that $\operatorname{tr}_{\varrho}(g)$ is not rational but

$$
\operatorname{tr}_{\varrho}(g), \operatorname{tr}_{\varrho^{*}}(g) \in \mathbb{Q}(\sqrt{-n})
$$

and $n$ divides $\operatorname{ord}(g)$.

The list of integers $n$ satisfying the two conditions of Proposition 7 is given in Table 10 . We omit from the table lambencies of Niemeier root systems for which there exists no integer $n$ satisfying these conditions.

From now on, we say that an irreducible representation $\varrho$ of the umbral group $G^{X}$ is of type $n$ if $n$ is an integer satisfying the two conditions of Proposition 7 and the character values of $\varrho$ generate the field $\mathbb{Q}(\sqrt{-n})$. Evidently, irreducible representations of type $n$ come in pairs $\left(\varrho, \varrho^{*}\right)$ with $\operatorname{tr}_{\varrho^{*}}(g)$ the complex conjugate of $\operatorname{tr}_{\varrho}(g)$ for all $g \in G^{X}$. The list of all irreducible representations of type $n$ is also given in Table 10. (See 'Irreducible characters' in Appendix 2 for the character tables of the $G^{X}$ and our notation for irreducible representations). 
Table 10 The irreducible representations of type $n$

\begin{tabular}{lll}
\hline $\boldsymbol{X}$ & $\boldsymbol{n}$ & $\left(\varrho, \varrho^{*}\right)$ \\
\hline$A_{1}^{24}$ & $7,15,23$ & $\left(\chi_{3}, \chi_{4}\right),\left(\chi_{5}, \chi_{6}\right),\left(\chi_{10}, \chi_{11}\right),\left(\chi_{12}, \chi_{13}\right),\left(\chi_{15}, \chi_{16}\right)$ \\
$A_{2}^{12}$ & $5,8,11,20$ & $\left(\chi_{4}, \chi_{5}\right),\left(\chi_{16}, \chi_{17}\right),\left(\chi_{20}, \chi_{21}\right),\left(\chi_{22}, \chi_{23}\right),\left(\chi_{25}, \chi_{26}\right)$ \\
$A_{3}^{8}$ & 3,7 & $\left(\chi_{2}, \chi_{3}\right),\left(\chi_{13}, \chi_{14}\right),\left(\chi_{15}, \chi_{16}\right)$ \\
$A_{4}^{6}$ & 4 & $\left(\chi_{8}, \chi_{9}\right),\left(\chi_{10}, \chi_{11}\right),\left(\chi_{12}, \chi_{13}\right)$ \\
$A_{5}^{4} D_{4}$ & 8 & $\left(\chi_{6}, \chi_{7}\right)$ \\
$A_{6}^{4}$ & 3 & $\left(\chi_{2}, \chi_{3}\right),\left(\chi_{6}, \chi_{7}\right)$ \\
$A_{9}^{2} D_{6}$ & 4 & $\left(\chi_{3}, \chi_{4}\right)$ \\
$A_{12}^{2}$ & 4 & $\left(\chi_{3}, \chi_{4}\right)$ \\
$D_{4}^{6}$ & 15 & $\left(\chi_{12}, \chi_{13}\right)$ \\
$E_{6}^{4}$ & 8 & $\left(\chi_{6}, \chi_{7}\right)$ \\
\hline
\end{tabular}

Recall that the Frobenius-Schur indicator of an irreducible ordinary representation of a finite group is $1,-1$ or 0 accordingly as the representation admits an invariant symmetric bilinear form, an invariant skew-symmetric bilinear form or no invariant bilinear form, respectively. The representations admitting no invariant bilinear form are precisely those whose character values are not all real. We can now state the next observation.

Proposition 8. For each Niemeier root system $X$, an irreducible representation $\varrho$ of $G^{X}$ has Frobenius-Schur indicator 0 if and only if it is of type $n$ for some $n$ defined in Proposition 7 .

The Schur index of an irreducible representation $\varrho$ of a finite group $G$ is the smallest positive integer $s$ such that there exists a degree $s$ extension $k$ of the field generated by the character values $\operatorname{tr}_{\varrho}(g)$ for $g \in G$ such that $\varrho$ can be realised over $k$. Inspired by Proposition 8, we make the following conjecture.

Conjecture 6. If $\varrho$ is an irreducible representation of $G^{X}$ of type $n$, then the Schur index of $\varrho$ is equal to 1.

In other words, we conjecture that the irreducible $G^{X}$-representations of type $n$ can be realised over $\mathbb{Q}(\sqrt{-n})$. For $X=A_{1}^{24}$, this speculation is in fact a theorem, since it is known [55] that the Schur indices for $M_{24}$ are always 1 . For $X=A_{2}^{12}$, it is also known [55] that the Schur indices for $\bar{G}^{(3)}=M_{12}$ are also always 1 . Moreover, the representations of $G^{(3)} \simeq 2 . \bar{G}^{(3)}$ with characters $\chi_{16}$ and $\chi_{17}$ in the notation in Appendix 2 have been constructed explicitly over $\mathbb{Q}(\sqrt{-2})$ in [56]. Finally, Proposition 8 constitutes a non-trivial consistency check for Conjecture 6 since the Schur index is at least 2 for a representation with Frobenius-Schur indicator equal to -1 .

Equipped with the preceding discussion, we are now ready to state our main observation for the discriminant property of umbral moonshine.

Proposition 9. Let $X$ be a Niemeier root system with Coxeter number $m$. Let $n$ be one of the integers in Table 10 and let $\lambda_{n}$ be the smallest positive integer such that $D=-n \lambda_{n}^{2}$ is a discriminant of $H^{X}$. Then, $K_{r,-D / 4 m}^{X}=\varrho_{n} \oplus \varrho_{n}^{*}$ where $\varrho_{n}$ and $\varrho_{n}^{*}$ are dual irreducible representations of type $n$. Conversely, if $\varrho$ is an irreducible representation of type $n$ and $-D$ is the smallest positive integer such that $K_{r,-D / 4 m}^{X}$ has $\varrho$ as an irreducible constituent, then there exists an integer $\lambda$ such that $D=-n \lambda^{2}$. 
Extending this, we make the following conjecture.

Conjecture 7. Let $X$ be a Niemeier root system with Coxeter number $m$. If $D$ is a discriminant of $H^{X}$ which satisfies $D=-n \lambda^{2}$ for some integer $\lambda$, then the representation $K_{r,-D / 4 m}^{X}$ has at least one dual pair of irreducible representations of type $n$ arising as irreducible constituents.

Conjecture (7) has been verified for the case $X=A_{1}^{24}$ in [57].

We conclude this section with conjectures arising from the observation ( $c f$. Additional file 2) that the conjectural $G^{X}$-module $K_{r, d}^{X}$ is typically isomorphic to several copies of a single representation. We say a $G$-module $V$ is a doublet if it is isomorphic to the direct sum of two copies of a single representation of $G$, and interpret the term sextet similarly.

Conjecture 8. Let $X$ be a Niemeier root system and let $m$ be the Coxeter number of $X$. Then, the representation $K_{r,-D / 4 m}^{X}$ is a doublet if and only if $D \neq 0$ and $D \neq-n \lambda^{2}$ for any integer $\lambda$ and for any $n$ listed in Table 10 corresponding to $X$. If $X=A_{8}^{3}$ then the representation $K_{r,-D / 4 m}^{X}$ is a sextet if and only if $D \neq-27 \lambda^{2}$ for some integer $\lambda$.

In particular, for the nine Niemeier root systems

$$
A_{7}^{2} D_{5}^{2}, A_{11} D_{7} E_{6}, D_{6}^{4}, D_{8}^{3}, D_{10} E_{7}^{2}, D_{12}^{2}, D_{16} E_{8}, D_{24}, E_{8}^{3},
$$

that have no irreducible representation with vanishing Forbenius-Schur indicator and have no terms with zero discriminant, we conjecture that all the representations $K_{r,-D / 4 m}^{X}$ corresponding to $H_{g}^{X}$ are doublets.

\section{Conclusions and discussion}

Let us start by recapitulating the main results of this paper. Taking the 23 Niemeier lattices as the starting point, in the 'Umbral groups' section, we identify a finite group $G^{X}$ - the umbral group - for each Niemeier root system $X$. On the other hand, using the ADE classification discussed in the 'The Eichler-Zagier operators and an ADE classification' section and Theorem 2, we identify a distinguished vector-valued mock modular form $H^{X}$ - the umbral form - for each Niemeier root system $X$. We then conjecture (among other things)

1. (Conjecture 1) that the mock modular form $H^{X}$ encodes the graded super-dimension of a certain infinite-dimensional, $\mathbb{Z} / 2 m \mathbb{Z} \times \mathbb{Q}$-graded module $K^{X}$ for $G^{X}$,

2. (Conjecture 3) that the graded super-characters $H_{g}^{X}$ arising from the action of $G^{X}$ on $K^{X}$ are vector-valued mock modular forms with concretely specified shadows $S_{g}^{X}$, and

3. (Conjecture 5) that the umbral McKay-Thompson series $H_{g}^{X}$ are uniquely determined by an optimal growth property which is directly analogous to the genus zero property of monstrous moonshine.

To lend evidence in support of these conjectures, we explicitly identify (almost all of) the umbral McKay-Thompson series $H_{g}^{X}$.

In this way, from the 23 Niemeier root systems, we obtain 23 instances of umbral moonshine, encompassing all the six instances previously discussed in [1] and in particular the case with $G^{X}=M_{24}$ first discussed in the context of the $K 3$ elliptic genus [11]. Apart 
from uncovering 17 new instances, we believe that the relation to Niemeier lattices sheds important light on the underlying structure of umbral moonshine. First, the construction of the umbral group $G^{X}$ is now completely uniform: $G^{X}$ is the outer-automorphism group of the corresponding Niemeier lattice (cf. (32)). Second, it provides an explanation for why the six instances discussed in [1] are naturally labelled by the divisors of 12: they correspond to Niemeier root systems given by evenly many copies (viz., 24/( $\ell-1)$ ) of an A-type root system $A_{\ell-1}$. Third, it also sheds light on the relation between umbral moonshine and meromorphic weight 1 Jacobi forms as well as weight 0 Jacobi forms. For as we have seen in the 'From Meromorphic Jacobi forms to mock modular forms' section, the umbral forms $H^{X}$ can be constructed uniformly by taking theta coefficients of finite parts of certain weight 1 meromorphic Jacobi forms, but in general, the relevant meromorphic Jacobi form has simple poles not only at the origin but also at non-trivial torsion points whenever the corresponding root system has a D- or E-type root system as an irreducible component. As a result, in those cases, the relation to weight 0 Jacobi forms is less direct as the Eichler-Zagier operator $\mathcal{W}^{X}$ of (136) is no longer proportional to the identity. In particular, in these cases, the umbral mock modular form $H^{X}$ does not arise in a direct way from the decomposition of a weight 0 (weak) Jacobi form into irreducible characters for the $\mathcal{N}=4$ superconformal algebra.

Recall that the relevant weight 0 Jacobi form in the construction described in the 'From weight 0 Jacobi forms to umbral mock modular forms' section coincides with the elliptic genus of a $K 3$ surface in the case of the Niemeier root system $X=A_{1}^{24}\left(\ell=2, G^{X}=\right.$ $M_{24}$ ). As the relation to weight 0 forms becomes less straightforward in the more general cases, the relation between umbral moonshine and sigma models, or in fact any kind of conformal field theory, also becomes more opaque. An interesting question is therefore the following. What, if any, kind of physical theory or geometry should attach to the more general instances of umbral moonshine?

To add to this puzzle, the Borcherds lift of the $K 3$ elliptic genus is a Siegel modular form which also plays an important role in type II as well as heterotic string theory compactified on $K 3 \times T^{2}$ [58-60]. As pointed out in [12] and refined in [17], Mathieu moonshine in this context (corresponding here to $X=A_{1}^{24}$ ) leads to predictions regarding Siegel modular forms which have been partially proven in [61]. Furthermore, this Siegel modular form also serves as the square of the denominator function of a generalised Kac-Moody algebra developed by Gritsenko-Nikulin in the context of mirror symmetry for $K 3$ surfaces [62-66]. As discussed in detail in [1], Section 5.5, many of these relations to string theory and $K 3$ geometry extend to some of the other five instances of umbral moonshine discussed in that paper. Since the relation between umbral forms and weight 0 modular forms is modified when D- or E-type root systems are involved, it would be extremely interesting to determine how the above-mentioned relations to string theory, $K 3$ surfaces and generalised Kac-Moody algebras manifest in the more general cases.

Regarding $K 3$ surfaces, note that Niemeier lattices have a long history of application to this field, and the study of the symmetries of $K 3$ surfaces in particular. See for instance [67]. See [68] for an analysis involving all of the Niemeier lattices. It would be interesting to explore the extent to which recent work $[22,23]$ applying the Niemeier lattice $L^{X}$ to the $X=A_{1}^{24}$ case of umbral moonshine can be extended to other Niemeier root systems in light of [68]. 
In another direction, the physical context of Mathieu moonshine has been extended recently to $K 3$ compactifications of heterotic string theory with eight supercharges [69]. As the structure of theories with 8 supercharges is much less rigid than those with 16 supercharges, one might speculate that a suitable generalisation of [69] could provide physical realisations of more instances of umbral moonshine.

Apart from posing the umbral moonshine conjecture, in this paper, we have also noted various intricate and mysterious properties of this new moonshine story. An important example is the close relation between Niemeier lattices and the genus zero groups discussed in the 'Genus zero groups' and 'From Niemeier lattices to principal moduli' sections. Another is the multiplicative relations discussed in the 'Multiplicative relations' section between McKay-Thompson series attached to different Niemeier root systems that plays an important role in the explicit specification of the umbral McKay-Thompson series in the 'Specification' section. While we have a concrete description of these two properties in terms of mock modular forms and related structures, their origin is still unclear. We also observe empirically discriminant relations between number fields underlying irreducible representations of $G^{X}$ and the discriminants of the vector-valued mock modular form $H^{X}$ ('Discriminants' section) extending the observations made in ([1], Section 5.4). It would be extremely interesting to have a representation theoretic explanation of these relations.

Last but not least, the construction of the umbral modules $K^{X}$ is clearly an important next step in unraveling the mystery of umbral moonshine.

\section{Endnote}

${ }^{a}$ We are grateful to George Glauberman for first alerting us to this fact.

\section{Appendix 1}

\section{Special functions}

\section{Dedekind Eta function}

The Dedekind eta function, denoted $\eta(\tau)$, is a holomorphic function on the upper halfplane defined by the infinite product

$$
\eta(\tau)=q^{1 / 24} \prod_{n \geq 1}\left(1-q^{n}\right)
$$

where $q=\mathrm{e}(\tau)=e^{2 \pi \mathbf{i} \tau}$. It is a modular form of weight $1 / 2$ for the modular group $S L_{2}(\mathbb{Z})$ with multiplier $\epsilon: S L_{2}(\mathbb{Z}) \rightarrow \mathbb{C}^{*}$, which means that

$$
\epsilon(\gamma) \eta(\gamma \tau) \operatorname{jac}(\gamma, \tau)^{1 / 4}=\eta(\tau)
$$

for all $\gamma=\left(\begin{array}{ll}a & b \\ c & d\end{array}\right) \in S L_{2}(\mathbb{Z})$, where $\operatorname{jac}(\gamma, \tau)=(c \tau+d)^{-2}$. The multiplier system $\epsilon$ may be described explicitly as

$$
\epsilon\left(\begin{array}{ll}
a & b \\
c & d
\end{array}\right)= \begin{cases}\mathrm{e}(-b / 24), & c=0, d=1 \\
\mathrm{e}(-(a+d) / 24 c+s(d, c) / 2+1 / 8), & c>0\end{cases}
$$

where $s(d, c)=\sum_{m=1}^{c-1}(d / c)((m d / c))$ and $((x))$ is 0 for $x \in \mathbb{Z}$ and $x-\lfloor x\rfloor-1 / 2$ otherwise. We can deduce the values $\epsilon(a, b, c, d)$ for $c<0$, or for $c=0$ and $d=-1$, by observing that $\epsilon(-\gamma)=\epsilon(\gamma) \mathrm{e}(1 / 4)$ for $\gamma \in S L_{2}(\mathbb{Z})$. 


\section{Jacobi theta functions}

We define the Jacboi theta functions $\theta_{i}(\tau, z)$ as follows for $q=e(\tau)$ and $y=e(z)$.

$$
\begin{aligned}
& \theta_{1}(\tau, z)=-i q^{1 / 8} y^{1 / 2} \prod_{n=1}^{\infty}\left(1-q^{n}\right)\left(1-y q^{n}\right)\left(1-y^{-1} q^{n-1}\right) \\
& \theta_{2}(\tau, z)=q^{1 / 8} y^{1 / 2} \prod_{n=1}^{\infty}\left(1-q^{n}\right)\left(1+y q^{n}\right)\left(1+y^{-1} q^{n-1}\right) \\
& \theta_{3}(\tau, z)=\prod_{n=1}^{\infty}\left(1-q^{n}\right)\left(1+y q^{n-1 / 2}\right)\left(1+y^{-1} q^{n-1 / 2}\right) \\
& \theta_{4}(\tau, z)=\prod_{n=1}^{\infty}\left(1-q^{n}\right)\left(1-y q^{n-1 / 2}\right)\left(1-y^{-1} q^{n-1 / 2}\right)
\end{aligned}
$$

Note that there are competing conventions for $\theta_{1}(\tau, z)$ in the literature and our normalisation may differ from another by a factor of -1 (or possibly $\pm i$ ).

\section{Higher level modular forms}

The congruence subgroups of the modular group $S L_{2}(\mathbb{Z})$ that are most relevant for this paper are the Hecke congruence groups

$$
\Gamma_{0}(N)=\left\{\left[\begin{array}{ll}
a & b \\
c & d
\end{array}\right] \in S L_{2}(\mathbb{Z}), c=0 \bmod N\right\} .
$$

A modular form for $\Gamma_{0}(N)$ is said to have level $N$. For $N$, a positive integer, a modular form of weight 2 for $\Gamma_{0}(N)$ is given by

$$
\begin{aligned}
\lambda_{N}(\tau) & =q \frac{d}{d q} \log \left(\frac{\eta(N \tau)}{\eta(\tau)}\right) \\
& =\frac{N-1}{24}+\sum_{k>0} \sigma(k)\left(q^{k}-N q^{N k}\right)
\end{aligned}
$$

where $\sigma(k)$ is the divisor function $\sigma(k)=\sum_{d \mid k} d$. The function $\lambda_{N}$ is, of course, only non-zero when $N>1$.

Observe that a modular form on $\Gamma_{0}(N)$ is a modular form on $\Gamma_{0}(M)$ whenever $N \mid M$, and for some small $N$, the space of forms of weight 2 is spanned by the $\lambda_{d}(\tau)$ for $d$, a divisor of $N$.

A discussion of the ring of weak Jacobi forms of higher level can be found in [70].

\section{Weight 0 Jacobi forms}

According to [71], the graded ring $J_{0, *}=\bigoplus_{m \geq 1} J_{0, m-1}$, of weak Jacobi forms with weight 0 and integral index ( $c f$. 'Jacobi forms' section), is finitely generated by $\varphi_{1}^{(2)}, \varphi_{1}^{(3)}$ and $\varphi_{1}^{(4)}$, where

$$
\begin{aligned}
& \varphi_{1}^{(2)}=4\left(f_{2}^{2}+f_{3}^{2}+f_{4}^{2}\right), \\
& \varphi_{1}^{(3)}=2\left(f_{2}^{2} f_{3}^{2}+f_{3}^{2} f_{4}^{2}+f_{4}^{2} f_{2}^{2}\right), \\
& \varphi_{1}^{(4)}=4 f_{2}^{2} f_{3}^{2} f_{4}^{2},
\end{aligned}
$$

and $f_{i}(\tau, z)=\theta_{i}(\tau, z) / \theta_{i}(\tau, 0)$ for $i \in\{2,3,4\}$ ( $c f$. 'Jacobi theta functions' section). If we work over $\mathbb{Z}$, then we must include

$$
\varphi_{1}^{(5)}=\frac{1}{4}\left(\varphi_{1}^{(4)} \varphi_{1}^{(2)}-\left(\varphi_{1}^{(3)}\right)^{2}\right)
$$


as a generator also, so that $J_{0, *}^{\mathbb{Z}}=\mathbb{Z}\left[\varphi_{1}^{(2)}, \varphi_{1}^{(3)}, \varphi_{1}^{(4)}, \varphi_{1}^{(5)}\right]$. The ring $J_{0, *}$ has an ideal

$$
J_{0, *}(q)=\bigoplus_{m>1} J_{0, m-1}(q)=\left\{\phi \in J_{0, *} \mid \phi(\tau, z)=\sum_{\substack{n, r \in \mathbb{Z} \\ n u>0}} c(n, r) q^{n} y^{r}\right\}
$$

consisting of Jacobi forms that vanish in the limit as $\tau \rightarrow i \infty$ (i.e. have vanishing coefficient of $q^{0} y^{r}$, for all $r$, in their Fourier expansion). This ideal is principal and generated by a weak Jacobi form of weight 0 and index 6 given by

$$
\zeta(\tau, z)=\frac{\theta_{1}(\tau, z)^{12}}{\eta(\tau)^{12}}
$$

( $c f$. 'Jacobi theta functions' section for $\theta_{1}$ and $\eta$ ). Gritsenko shows [71] that for any positive integer $m$, the quotient $J_{0, m-1} / J_{0, m-1}(q)$ is a vector space of dimension $m-1$ admitting a basis consisting of weight 0 index $m-1$ weak Jacobi forms $\varphi_{n}^{(m)}\left(\operatorname{denoted} \psi_{0, m-1}^{(n)}\right.$ in [71]) for $1 \leq n \leq m-1$ such that the coefficient of $q^{0} y^{k}$ in $\varphi_{n}^{(m)}$ vanishes for $|k|>n$ but does not vanish for $|k|=n$. In fact, Gritsenko works in the subring $J_{0, *}^{\mathbb{Z}}$ of Jacobi forms having integer Fourier coefficients and his $\varphi_{n}^{(m)}$ furnish a $\mathbb{Z}$-basis for the $\mathbb{Z}$-module $J_{0, *}^{\mathbb{Z}} / J_{0, *}^{\mathbb{Z}}(q)$. In what follows, we record explicit formulas for some of the $\varphi_{n}^{(m)}$.

Following [71], we define

$$
\begin{gathered}
\varphi_{1}^{(7)}=\varphi_{1}^{(3)} \varphi_{1}^{(5)}-\left(\varphi_{1}^{(4)}\right)^{2}, \\
\varphi_{1}^{(9)}=\varphi_{1}^{(3)} \varphi_{1}^{(7)}-\left(\varphi_{1}^{(5)}\right)^{2}, \\
\varphi_{1}^{(13)}=\varphi_{1}^{(5)} \varphi_{1}^{(9)}-2\left(\varphi_{1}^{(7)}\right)^{2},
\end{gathered}
$$

and define $\varphi_{1}^{(m)}$ for the remaining positive integers $m$ according to the following recursive procedure. For $(12, m-1)=1$ and $m>5$, we set

$$
\varphi_{1}^{(m)}=(12, m-5) \varphi_{1}^{(m-4)} \varphi_{1}^{(5)}+(12, m-3) \varphi_{1}^{(m-2)} \varphi_{1}^{(3)}-2(12, m-4) \varphi_{1}^{(m-3)} \varphi_{1}^{(4)} .
$$

For $(12, m-1)=2$ and $m>10$, we set

$$
\varphi_{1}^{(m)}=\frac{1}{2}\left((12, m-5) \varphi_{1}^{(m-4)} \varphi_{1}^{(5)}+(12, m-3) \varphi_{1}^{(m-2)} \varphi_{1}^{(3)}-2(12, m-4) \varphi_{1}^{(m-3)} \varphi_{1}^{(4)}\right) .
$$

For $(12, m-1)=3$ and $m>9$, we set

$$
\varphi_{1}^{(m)}=\frac{2}{3}(12, m-4) \varphi_{1}^{(m-3)} \varphi_{1}^{(4)}+\frac{1}{3}(12, m-7) \varphi_{1}^{(m-6)} \varphi_{1}^{(7)}-(12, m-5) \varphi_{1}^{(m-4)} \varphi_{1}^{(5)} .
$$

For $(12, m-1)=4$ and $m>16$, we set

$$
\varphi_{1}^{(m)}=\frac{1}{4}\left((12, m-13) \varphi_{1}^{(m-12)} \varphi_{1}^{(13)}+(12, m-5) \varphi_{1}^{(m-4)} \varphi_{1}^{(5)}-(12, m-9) \varphi_{1}^{(m-8)} \varphi_{1}^{(9)}\right) .
$$

For $(12, m-1)=6$ and $m>18$, we set

$$
\varphi_{1}^{(m)}=\frac{1}{3}(12, m-4) \varphi_{1}^{(m-3)} \varphi_{1}^{(4)}+\frac{1}{6}(12, m-7) \varphi_{1}^{(m-6)} \varphi_{1}^{(7)}-\frac{1}{2}(12, m-5) \varphi_{1}^{(m-4)} \varphi_{1}^{(5)} .
$$

Finally, for $(12, m-1)=12$ and $m>24$, we set

$$
\varphi_{1}^{(m)}=\frac{1}{6}(12, m-4) \varphi_{1}^{(m-3)} \varphi_{1}^{(4)}-\frac{1}{4}(12, m-5) \varphi_{1}^{(m-4)} \varphi_{1}^{(5)}+\frac{1}{12}(12, m-7) \varphi_{1}^{(m-6)} \varphi_{1}^{(7)},
$$


where we have set

$$
\varphi_{1}^{(25)}=\frac{1}{2} \varphi_{1}^{(21)} \varphi_{1}^{(5)}-\varphi_{1}^{(19)} \varphi_{1}^{(7)}+\frac{1}{2}\left(\varphi_{1}^{(13)}\right)^{2} .
$$

The $\varphi_{2}^{(m)}$ are defined by setting

$$
\begin{aligned}
& \varphi_{2}^{(3)}=\left(\varphi_{1}^{(2)}\right)^{2}-24 \varphi_{1}^{(3)}, \\
& \varphi_{2}^{(4)}=\varphi_{1}^{(2)} \varphi_{1}^{(3)}-18 \varphi_{1}^{(4)}, \\
& \varphi_{2}^{(5)}=\varphi_{1}^{(2)} \varphi_{1}^{(4)}-16 \varphi_{1}^{(5)},
\end{aligned}
$$

and

$$
\varphi_{2}^{(m)}=(12, m-4) \varphi_{1}^{(m-3)} \varphi_{1}^{(4)}-(12, m-5) \varphi_{1}^{(m-4)} \varphi_{1}^{(5)}-(12, m-1) \varphi_{1}^{(m)}
$$

for $m>5$, and the remaining $\varphi_{n}^{(m)}$ for $2 \leq m \leq 25$ are given by

$$
\begin{aligned}
\varphi_{n}^{(m)} & =\varphi_{n-1}^{(m-3)} \varphi_{1}^{(4)}, \\
\varphi_{m-2}^{(m)} & =\left(\varphi_{1}^{(2)}\right)^{m-3} \varphi_{1}^{(3)}, \\
\varphi_{m-1}^{(m)} & =\left(\varphi_{1}^{(2)}\right)^{m-1},
\end{aligned}
$$

where the first equation of (252) holds for $3 \leq n \leq m-3$.

\section{Appendix 2}

\section{Characters}

In 'Irreducible characters' we give character tables (with power maps and FrobeniusSchur indicators) for each group $G^{X}$ for $X$ a Niemeier root system. These were computed with the aid of the computer algebra package GAP4 [72]. We use the abbreviations $a_{n}=\sqrt{-n}$ and $b_{n}=(-1+\sqrt{-n}) / 2$ in these tables.

The tables in 'Euler characters' furnish cycle shapes and character values - the twisted Euler characters - attached to the representations of the groups $G^{X}$ described in the 'Umbral groups' section. Using this data, we can obtain explicit expressions for the shadows $S_{g}^{X}$ of the vector-valued mock modular forms $H_{g}^{X}$ according to the prescription of the 'Shadows' section.

Irreducible characters (Tables 11, 12, 13, 14, 15, 16, 17, 18, 19, 20, 21, 22 and 23)

\section{Euler characters}

The tables in this section describe the twisted Euler characters and associated cycle shapes attached to each group $G^{X}$ in the 'Umbral groups' section (Tables 24, 25, 26, 27, 28, $29,30,31,32,33,34,35,36,37,38,39,40,41,42,43$ and 44). According to the prescription of the 'Shadows' section, the character values $\bar{\chi}_{g}^{X_{A}}, \chi_{g}^{X_{A}}$, \&c., can be used to describe the shadows of the vector-valued mock modular forms $H_{g}^{X}$ attached to each $g \in G^{X}$ by umbral moonshine. We also identify symbols $n_{g} \mid h_{g}$ which are used in the 'Modularity' section to formulate conjectures about the modularity of $H_{g}^{X}$. By definition, $n_{g}$ is the order of the image of $g \in G^{X}$ in $\bar{G}^{X}$ and $h_{g}=N_{g} / n_{g}$ where $N_{g}$ denotes the product of shortest and longest cycle lengths appearing in the cycle shape $\widetilde{\Pi}_{g}^{X}$.

Note that we have $\widetilde{\Pi}_{g}^{X}=\widetilde{\Pi}_{g}^{X_{A}}=\bar{\Pi}_{g}^{X_{A}}$ in case $X=A_{1}^{24}$. More generally, we will have $\widetilde{\Pi}_{g}^{X}=\widetilde{\Pi}_{g}^{X_{A}}$ when $X=X_{A}$ (cf. 'Lattices' section), and similarly when $X=X_{D}$ or $X=X_{E}$, so we suppress the row (that would otherwise be) labelled $\widetilde{\Pi}_{g}^{X}$ in these cases. 
Table 11 Character table of $G^{X} \simeq M_{24}, X=A_{1}^{24}$

\begin{tabular}{|c|c|c|c|c|c|c|c|c|c|c|c|c|c|c|c|c|c|c|c|c|c|c|c|c|c|c|c|}
\hline$[g]$ & FS & $1 \mathrm{~A}$ & $2 A$ & $2 B$ & $3 \mathrm{~A}$ & $3 B$ & $4 \mathrm{~A}$ & 4B & $4 C$ & $5 \mathrm{~A}$ & $6 \mathrm{~A}$ & $6 \mathrm{~B}$ & $7 \mathrm{~A}$ & 7B & $8 \mathrm{~A}$ & $10 \mathrm{~A}$ & $11 \mathrm{~A}$ & $12 \mathrm{~A}$ & $12 \mathrm{~B}$ & $14 \mathrm{~A}$ & 14B & $15 \mathrm{~A}$ & 15B & $21 \mathrm{~A}$ & 21B & $23 \mathrm{~A}$ & $23 \mathrm{~B}$ \\
\hline$\left[g^{2}\right]$ & & $1 \mathrm{~A}$ & $1 \mathrm{~A}$ & $1 \mathrm{~A}$ & $3 A$ & 3B & $2 A$ & $2 A$ & $2 B$ & $5 A$ & $3 A$ & $3 B$ & $7 A$ & $7 B$ & 4B & $5 A$ & $11 \mathrm{~A}$ & $6 \mathrm{~A}$ & $6 \mathrm{~B}$ & 7A & 7B & $15 \mathrm{~A}$ & $15 B$ & $21 \mathrm{~A}$ & 21B & $23 \mathrm{~A}$ & $23 B$ \\
\hline$\left[g^{3}\right]$ & & $1 \mathrm{~A}$ & $2 A$ & $2 B$ & $1 \mathrm{~A}$ & $1 \mathrm{~A}$ & $4 A$ & 4B & $4 C$ & $5 A$ & $2 A$ & $2 B$ & 7B & $7 A$ & $8 \mathrm{~A}$ & $10 \mathrm{~A}$ & $11 \mathrm{~A}$ & $4 A$ & $4 C$ & 14B & $14 \mathrm{~A}$ & $5 A$ & $5 A$ & 7B & $7 A$ & $23 A$ & $23 B$ \\
\hline$\left[g^{5}\right]$ & & $1 \mathrm{~A}$ & $2 A$ & $2 B$ & $3 A$ & $3 B$ & $4 \mathrm{~A}$ & 4B & $4 C$ & $1 \mathrm{~A}$ & $6 \mathrm{~A}$ & $6 B$ & $7 B$ & $7 A$ & $8 \mathrm{~A}$ & $2 B$ & $11 \mathrm{~A}$ & $12 \mathrm{~A}$ & $12 B$ & $14 \mathrm{~B}$ & $14 \mathrm{~A}$ & $3 A$ & $3 A$ & $21 B$ & $21 \mathrm{~A}$ & $23 B$ & $23 \mathrm{~A}$ \\
\hline$\left[g^{7}\right]$ & & $1 \mathrm{~A}$ & $2 A$ & $2 B$ & $3 A$ & $3 B$ & $4 A$ & 4B & $4 C$ & $5 A$ & $6 \mathrm{~A}$ & $6 B$ & $1 \mathrm{~A}$ & $1 \mathrm{~A}$ & $8 \mathrm{~A}$ & $10 \mathrm{~A}$ & $11 \mathrm{~A}$ & $12 \mathrm{~A}$ & $12 \mathrm{~B}$ & $2 A$ & $2 A$ & $15 B$ & $15 \mathrm{~A}$ & $3 B$ & 3B & $23 B$ & $23 \mathrm{~A}$ \\
\hline$\left[g^{11}\right]$ & & $1 \mathrm{~A}$ & $2 A$ & $2 B$ & $3 A$ & 3B & $4 \mathrm{~A}$ & 4B & $4 C$ & $5 A$ & $6 \mathrm{~A}$ & $6 B$ & $7 A$ & 7B & $8 \mathrm{~A}$ & $10 \mathrm{~A}$ & $1 \mathrm{~A}$ & $12 \mathrm{~A}$ & $12 B$ & $14 \mathrm{~A}$ & $14 B$ & $15 B$ & $15 \mathrm{~A}$ & $21 \mathrm{~A}$ & 21B & $23 B$ & $23 A$ \\
\hline$\left[g^{23}\right]$ & & $1 \mathrm{~A}$ & $2 A$ & $2 B$ & $3 A$ & $3 B$ & $4 A$ & 4B & $4 C$ & $5 \mathrm{~A}$ & $6 \mathrm{~A}$ & $6 \mathrm{~B}$ & $7 \mathrm{~A}$ & $7 B$ & $8 \mathrm{~A}$ & $10 \mathrm{~A}$ & $11 \mathrm{~A}$ & $12 \mathrm{~A}$ & $12 \mathrm{~B}$ & $14 \mathrm{~A}$ & 14B & $15 \mathrm{~A}$ & $15 \mathrm{~B}$ & $21 \mathrm{~A}$ & 21B & $1 \mathrm{~A}$ & $1 \mathrm{~A}$ \\
\hline$\chi_{1}$ & + & 1 & 1 & 1 & 1 & 1 & 1 & 1 & 1 & 1 & 1 & 1 & 1 & 1 & 1 & 1 & 1 & 1 & 1 & 1 & 1 & 1 & 1 & 1 & 1 & 1 & 1 \\
\hline$\chi_{2}$ & + & 23 & 7 & -1 & 5 & -1 & -1 & 3 & -1 & 3 & 1 & -1 & 2 & 2 & 1 & -1 & 1 & -1 & -1 & 0 & 0 & ( & 0 & -1 & -1 & 0 & 0 \\
\hline$\chi_{3}$ & $\circ$ & 45 & -3 & 5 & 0 & 3 & -3 & 1 & 1 & 0 & 0 & -1 & $\underline{b_{7}}$ & $\overline{b_{7}}$ & -1 & 0 & 1 & 0 & 1 & $-b_{7}$ & $-\overline{b_{7}}$ & 0 & 0 & $\underline{b_{7}}$ & $\overline{b_{7}}$ & -1 & -1 \\
\hline$\chi_{4}$ & $\circ$ & 45 & -3 & 5 & 0 & 3 & -3 & 1 & 1 & 0 & 0 & -1 & $\overline{b_{7}}$ & $b_{7}$ & -1 & 0 & 1 & 0 & 1 & $-\overline{b_{7}}$ & $-b_{7}$ & 0 & 0 & $\overline{b_{7}}$ & $b_{7}$ & -1 & -1 \\
\hline$\chi_{5}$ & $\circ$ & 231 & 7 & -9 & -3 & 0 & -1 & -1 & 3 & 1 & 1 & 0 & 0 & 0 & - & 1 & 0 & -1 & 0 & 0 & 0 & $b_{15}$ & $\overline{b_{15}}$ & 0 & 0 & 1 & 1 \\
\hline$\chi_{6}$ & $\circ$ & 231 & 7 & -9 & -3 & 0 & -1 & -1 & 3 & 1 & 1 & 0 & 0 & 0 & -1 & 1 & 0 & -1 & 0 & 0 & 0 & $\overline{b_{15}}$ & $b_{15}$ & 0 & 0 & 1 & 1 \\
\hline$\chi_{7}$ & + & 252 & 28 & 12 & 9 & 0 & 4 & 4 & 0 & 2 & 1 & 0 & 0 & 0 & 0 & 2 & -1 & 1 & 0 & 0 & 0 & -1 & -1 & 0 & 0 & -1 & -1 \\
\hline$\chi_{8}$ & + & 253 & 13 & -11 & 10 & 1 & -3 & 1 & 1 & 3 & -2 & 1 & 1 & 1 & -1 & -1 & 0 & 0 & 1 & -1 & -1 & 0 & 0 & 1 & 1 & 0 & 0 \\
\hline$\chi_{9}$ & + & 483 & 35 & 3 & 6 & 0 & 3 & 3 & 3 & -2 & 2 & 0 & 0 & 0 & -1 & -2 & -1 & 0 & 0 & 0 & 0 & 1 & 1 & 0 & 0 & 0 & 0 \\
\hline$\chi_{10}$ & $\circ$ & 770 & -14 & 10 & 5 & -7 & 2 & -2 & -2 & 0 & 1 & 1 & 0 & 0 & 0 & 0 & 0 & -1 & 1 & 0 & 0 & 0 & 0 & 0 & 0 & $\underline{b_{23}}$ & $\overline{b_{23}}$ \\
\hline$\chi_{11}$ & $\circ$ & 770 & -14 & 10 & 5 & -7 & 2 & -2 & -2 & 0 & 1 & 1 & 0 & $\underline{0}$ & 0 & 0 & 0 & -1 & 1 & 0 & 0 & 0 & 0 & 0 & $\underline{0}$ & $\overline{b_{23}}$ & $b_{23}$ \\
\hline$\chi_{12}$ & $\circ$ & 990 & -18 & -10 & 0 & 3 & 6 & 2 & -2 & 0 & 0 & -1 & $b_{7}$ & $\overline{b_{7}}$ & 0 & 0 & 0 & 0 & 1 & $\underline{b_{7}}$ & $\overline{b_{7}}$ & 0 & 0 & $\underline{b_{7}}$ & $\overline{b_{7}}$ & 1 & 1 \\
\hline$\chi_{13}$ & $\circ$ & 990 & -18 & -10 & 0 & 3 & 6 & 2 & -2 & 0 & 0 & -1 & $\overline{b_{7}}$ & $b_{7}$ & 0 & 0 & 0 & 0 & 1 & $\overline{b_{7}}$ & $b_{7}$ & 0 & 0 & $\overline{b_{7}}$ & $b_{7}$ & 1 & 1 \\
\hline$\chi_{14}$ & + & 1,035 & 27 & 35 & 0 & 6 & 3 & -1 & 3 & 0 & 0 & 2 & -1 & -1 & 1 & 0 & 1 & 0 & 0 & -1 & -1 & 0 & 0 & -1 & -1 & 0 & 0 \\
\hline$\chi_{15}$ & $\circ$ & 1,035 & -21 & -5 & 0 & -3 & 3 & 3 & -1 & 0 & 0 & 1 & $2 b_{7}$ & $2 \overline{b_{7}}$ & -1 & 0 & 1 & 0 & -1 & 0 & 0 & 0 & 0 & $-\underline{b_{7}}$ & $-\overline{b_{7}}$ & 0 & 0 \\
\hline$\chi_{16}$ & $\circ$ & 1,035 & -21 & -5 & 0 & -3 & 3 & 3 & -1 & 0 & 0 & 1 & $2 \overline{b_{7}}$ & $2 b_{7}$ & -1 & 0 & 1 & 0 & -1 & 0 & 0 & 0 & 0 & $-\overline{b_{7}}$ & $-b_{7}$ & 0 & 0 \\
\hline$\chi_{17}$ & + & 1,265 & 49 & -15 & 5 & 8 & -7 & 1 & -3 & 0 & 1 & 0 & -2 & -2 & 1 & 0 & 0 & -1 & 0 & 0 & 0 & 0 & 0 & 1 & 1 & 0 & 0 \\
\hline$\chi_{18}$ & + & 1,771 & -21 & 11 & 16 & 7 & 3 & -5 & -1 & 1 & 0 & -1 & 0 & 0 & -1 & 1 & ( & 0 & -1 & c & 0 & 1 & 1 & 0 & 0 & 0 & 0 \\
\hline$\chi_{19}$ & + & 2,024 & 8 & 22 & -1 & 8 & 8 & 0 & 0 & -1 & -1 & 0 & 1 & 1 & 0 & -1 & & -1 & 0 & 1 & 1 & -1 & -1 & 1 & 1 & 0 & 0 \\
\hline$\chi_{20}$ & + & 2,277 & 21 & -19 & 0 & 6 & -3 & 1 & -3 & -3 & 0 & 2 & 2 & 2 & -1 & 1 & & 0 & 0 & 0 & 0 & 0 & 0 & -1 & -1 & 0 & 0 \\
\hline$\chi_{21}$ & + & 3,312 & 48 & 16 & 0 & -6 & 0 & 0 & 0 & -3 & 0 & -2 & 1 & 1 & 0 & 1 & & 0 & 0 & -1 & -1 & 0 & 0 & 1 & 1 & 0 & 0 \\
\hline$\chi_{22}$ & + & 3,520 & 64 & 0 & 10 & -8 & 0 & 0 & 0 & 0 & -2 & 0 & -1 & -1 & 0 & 0 & & 0 & 0 & 1 & 1 & 0 & 0 & -1 & -1 & 1 & 1 \\
\hline$\chi_{23}$ & + & 5,313 & 49 & 9 & -15 & 0 & 1 & -3 & -3 & 3 & 1 & 0 & 0 & 0 & -1 & -1 & & I & 0 & 0 & 0 & 0 & 0 & 0 & 0 & 0 & 0 \\
\hline$\chi_{24}$ & + & 5,544 & -56 & 24 & 9 & 0 & -8 & 0 & 0 & -1 & 1 & 0 & 0 & 0 & 0 & -1 & 0 & 1 & 0 & 0 & 0 & -1 & -1 & 0 & 0 & 1 & 1 \\
\hline$\chi_{25}$ & + & 5,796 & -28 & 36 & -9 & 0 & -4 & 4 & 0 & 1 & -1 & 0 & 0 & 0 & 0 & 1 & -1 & -1 & 0 & 0 & 0 & 1 & 1 & 0 & 0 & 0 & 0 \\
\hline$\chi_{26}$ & + & 10,395 & -21 & -45 & 0 & 0 & 3 & -1 & 3 & 0 & 0 & 0 & 0 & 0 & 1 & 0 & 0 & 0 & 0 & 0 & 0 & 0 & 0 & 0 & 0 & -1 & -1 \\
\hline
\end{tabular}


Table 12 Character table of $G^{X} \simeq 2 . M_{12}, X=A_{2}^{12}$

\begin{tabular}{|c|c|c|c|c|c|c|c|c|c|c|c|c|c|c|c|c|c|c|c|c|c|c|c|c|c|c|c|}
\hline$[g]$ & FS & $1 \mathrm{~A}$ & $2 A$ & $4 \mathrm{~A}$ & $2 B$ & $2 C$ & $3 A$ & $6 \mathrm{~A}$ & $3 B$ & $6 \mathrm{~B}$ & 4B & $4 C$ & $5 A$ & $10 \mathrm{~A}$ & $12 \mathrm{~A}$ & $6 C$ & 6D & $8 \mathrm{~A}$ & $8 B$ & $8 C$ & $8 D$ & $20 \mathrm{~A}$ & $20 \mathrm{~B}$ & $11 \mathrm{~A}$ & $22 \mathrm{~A}$ & 11B & $22 B$ \\
\hline$\left[g^{2}\right]$ & & $1 \mathrm{~A}$ & $1 \mathrm{~A}$ & $2 A$ & $1 \mathrm{~A}$ & $1 \mathrm{~A}$ & $3 A$ & $3 A$ & 3B & $3 B$ & $2 B$ & $2 B$ & $5 \mathrm{~A}$ & $5 A$ & $6 \mathrm{~B}$ & $3 A$ & $3 A$ & 4B & 4B & $4 C$ & $4 C$ & $10 \mathrm{~A}$ & $10 \mathrm{~A}$ & 11B & 11B & $11 \mathrm{~A}$ & $\overline{11 \mathrm{~A}}$ \\
\hline$\left[g^{3}\right]$ & & $1 \mathrm{~A}$ & $2 A$ & $4 \mathrm{~A}$ & $2 B$ & $2 C$ & $1 \mathrm{~A}$ & $2 A$ & $1 \mathrm{~A}$ & $2 A$ & 4B & $4 C$ & $5 \mathrm{~A}$ & $10 \mathrm{~A}$ & $4 \mathrm{~A}$ & $2 B$ & $2 C$ & $8 \mathrm{~A}$ & $8 B$ & $8 C$ & $8 D$ & $20 \mathrm{~A}$ & $20 B$ & $11 \mathrm{~A}$ & $22 \mathrm{~A}$ & 11B & $22 B$ \\
\hline$\left[g^{5}\right]$ & & $1 \mathrm{~A}$ & $2 A$ & $4 \mathrm{~A}$ & $2 B$ & $2 C$ & $3 A$ & $6 \mathrm{~A}$ & 3B & $6 \mathrm{~B}$ & 4B & $4 C$ & $1 \mathrm{~A}$ & $2 A$ & $12 \mathrm{~A}$ & $6 C$ & $6 \mathrm{D}$ & $8 B$ & $8 \mathrm{~A}$ & $8 D$ & $8 C$ & $4 \mathrm{~A}$ & $4 \mathrm{~A}$ & $11 \mathrm{~A}$ & $22 \mathrm{~A}$ & $11 \mathrm{~B}$ & $22 B$ \\
\hline$\left[g^{11}\right]$ & & $1 \mathrm{~A}$ & $2 A$ & $4 \mathrm{~A}$ & $2 B$ & $2 C$ & $3 A$ & $6 \mathrm{~A}$ & $3 B$ & $6 \mathrm{~B}$ & 4B & $4 C$ & $5 \mathrm{~A}$ & $10 \mathrm{~A}$ & $12 \mathrm{~A}$ & $6 C$ & $6 \mathrm{D}$ & $8 \mathrm{~A}$ & $8 B$ & $8 C$ & $8 D$ & $20 B$ & $20 \mathrm{~A}$ & $1 \mathrm{~A}$ & $2 A$ & $1 \mathrm{~A}$ & $2 A$ \\
\hline$\chi_{1}$ & + & 1 & 1 & 1 & 1 & 1 & 1 & 1 & 1 & 1 & 1 & 1 & 1 & 1 & 1 & 1 & 1 & 1 & 1 & 1 & 1 & 1 & 1 & 1 & 1 & 1 & $\overline{1}$ \\
\hline$\chi_{2}$ & + & 11 & 11 & -1 & 3 & 3 & 2 & 2 & -1 & -1 & -1 & 3 & 1 & 1 & -1 & 0 & 0 & -1 & -1 & 1 & 1 & -1 & -1 & 0 & 0 & 0 & 0 \\
\hline$\chi_{3}$ & + & 11 & 11 & -1 & 3 & 3 & 2 & 2 & -1 & -1 & 3 & -1 & 1 & 1 & -1 & 0 & 0 & 1 & 1 & -1 & -1 & -1 & -1 & 0 & 0 & 0 & 0 \\
\hline$\chi_{4}$ & 。 & 16 & 16 & 4 & 0 & 0 & -2 & -2 & 1 & 1 & 0 & 0 & 1 & 1 & 1 & 0 & 0 & 0 & 0 & 0 & 0 & -1 & -1 & $b_{11}$ & $b_{11}$ & $\overline{b_{11}}$ & $\overline{b_{11}}$ \\
\hline$\chi_{5}$ & 。 & 16 & 16 & 4 & 0 & 0 & -2 & -2 & 1 & 1 & 0 & 0 & 1 & 1 & 1 & 0 & 0 & 0 & 0 & 0 & 0 & -1 & -1 & $\overline{b_{11}}$ & $\overline{b_{11}}$ & $b_{11}$ & $b_{11}$ \\
\hline$\chi_{6}$ & + & 45 & 45 & 5 & -3 & -3 & 0 & 0 & 3 & 3 & 1 & 1 & 0 & 0 & -1 & 0 & 0 & -1 & -1 & -1 & -1 & 0 & 0 & 1 & 1 & 1 & 1 \\
\hline$\chi_{7}$ & + & 54 & 54 & 6 & 6 & 6 & 0 & 0 & 0 & 0 & 2 & 2 & -1 & -1 & 0 & 0 & 0 & 0 & 0 & 0 & 0 & 1 & 1 & -1 & -1 & -1 & -1 \\
\hline$\chi_{8}$ & + & 55 & 55 & -5 & 7 & 7 & 1 & 1 & 1 & 1 & -1 & -1 & 0 & 0 & 1 & 1 & 1 & -1 & -1 & -1 & -1 & 0 & 0 & 0 & 0 & 0 & 0 \\
\hline$\chi_{9}$ & + & 55 & 55 & -5 & -1 & -1 & 1 & 1 & 1 & 1 & 3 & -1 & 0 & 0 & 1 & -1 & -1 & -1 & -1 & 1 & 1 & 0 & 0 & 0 & 0 & 0 & 0 \\
\hline$\chi_{10}$ & + & 55 & 55 & -5 & -1 & -1 & 1 & 1 & 1 & 1 & -1 & 3 & 0 & 0 & 1 & -1 & -1 & 1 & 1 & -1 & -1 & 0 & 0 & 0 & 0 & 0 & 0 \\
\hline$\chi_{11}$ & + & 66 & 66 & 6 & 2 & 2 & 3 & 3 & 0 & 0 & -2 & -2 & 1 & 1 & 0 & -1 & -1 & 0 & 0 & 0 & 0 & 1 & 1 & 0 & 0 & 0 & 0 \\
\hline$\chi_{12}$ & + & 99 & 99 & -1 & 3 & 3 & 0 & 0 & 3 & 3 & -1 & -1 & -1 & -1 & -1 & 0 & 0 & 1 & 1 & 1 & 1 & -1 & -1 & 0 & 0 & 0 & 0 \\
\hline$\chi_{13}$ & + & 120 & 120 & 0 & -8 & -8 & 3 & 3 & 0 & 0 & 0 & 0 & 0 & 0 & 0 & 1 & 1 & 0 & 0 & 0 & 0 & 0 & 0 & -1 & -1 & -1 & -1 \\
\hline$\chi_{14}$ & + & 144 & 144 & 4 & 0 & 0 & 0 & 0 & -3 & -3 & 0 & 0 & -1 & -1 & 1 & 0 & 0 & 0 & 0 & 0 & 0 & -1 & -1 & 1 & 1 & 1 & 1 \\
\hline$\chi_{15}$ & + & 176 & 176 & -4 & 0 & 0 & -4 & -4 & -1 & -1 & 0 & 0 & 1 & 1 & -1 & 0 & 0 & 0 & 0 & 0 & 0 & 1 & 1 & 0 & 0 & 0 & 0 \\
\hline$\chi_{16}$ & $\circ$ & 10 & -10 & 0 & -2 & 2 & 1 & -1 & -2 & 2 & 0 & 0 & 0 & 0 & 0 & 1 & -1 & $a_{2}$ & $\overline{a_{2}}$ & $a_{2}$ & $\overline{a_{2}}$ & 0 & 0 & -1 & 1 & -1 & 1 \\
\hline$\chi_{17}$ & ○ & 10 & -10 & 0 & -2 & 2 & 1 & -1 & -2 & 2 & 0 & 0 & 0 & 0 & 0 & 1 & -1 & $\frac{2}{a_{2}}$ & $a_{2}$ & $\overline{a_{2}}$ & $a_{2}$ & 0 & 0 & -1 & 1 & -1 & 1 \\
\hline$\chi_{18}$ & + & 12 & -12 & 0 & 4 & -4 & 3 & -3 & 0 & 0 & 0 & 0 & 2 & -2 & 0 & 1 & -1 & 0 & 0 & 0 & 0 & 0 & 0 & 1 & -1 & 1 & -1 \\
\hline$\chi_{19}$ & - & 32 & -32 & 0 & 0 & 0 & -4 & 4 & 2 & -2 & 0 & 0 & 2 & -2 & 0 & 0 & 0 & 0 & 0 & 0 & 0 & 0 & 0 & -1 & 1 & -1 & 1 \\
\hline$\chi_{20}$ & 。 & 44 & -44 & 0 & 4 & -4 & -1 & 1 & 2 & -2 & 0 & 0 & -1 & 1 & 0 & 1 & -1 & 0 & 0 & 0 & 0 & $a_{5}$ & $\overline{a_{5}}$ & 0 & 0 & 0 & 0 \\
\hline$\chi_{21}$ & $\circ$ & 44 & -44 & 0 & 4 & -4 & -1 & 1 & 2 & -2 & 0 & 0 & -1 & 1 & 0 & 1 & -1 & 0 & 0 & 0 & 0 & $\overline{a_{5}}$ & $a_{5}$ & 0 & 0 & 0 & 0 \\
\hline$\chi_{22}$ & $\circ$ & 110 & -110 & 0 & -6 & 6 & 2 & -2 & 2 & -2 & 0 & 0 & 0 & 0 & 0 & 0 & 0 & $a_{2}$ & $\overline{a_{2}}$ & $\overline{a_{2}}$ & $a_{2}$ & 0 & 0 & 0 & 0 & 0 & 0 \\
\hline$\chi_{23}$ & 。 & 110 & -110 & 0 & -6 & 6 & 2 & -2 & 2 & -2 & 0 & 0 & 0 & 0 & 0 & 0 & 0 & $\frac{z}{a_{2}}$ & $a_{2}$ & $a_{2}$ & $\frac{2}{a_{2}}$ & 0 & 0 & 0 & 0 & 0 & 0 \\
\hline$\chi_{24}$ & + & 120 & -120 & 0 & 8 & -8 & 3 & -3 & 0 & 0 & 0 & 0 & 0 & 0 & 0 & -1 & 1 & 0 & 0 & 0 & 0 & 0 & 0 & -1 & 1 & -1 & 1 \\
\hline$\chi_{25}$ & 。 & 160 & -160 & 0 & 0 & 0 & -2 & 2 & -2 & 2 & 0 & 0 & 0 & 0 & 0 & 0 & 0 & 0 & 0 & 0 & 0 & 0 & 0 & $-b_{11}$ & $b_{11}$ & $-\overline{b_{11}}$ & $\overline{b_{11}}$ \\
\hline$\chi_{26}$ & ○ & 160 & -160 & 0 & 0 & 0 & -2 & 2 & -2 & 2 & 0 & 0 & 0 & 0 & 0 & 0 & 0 & 0 & 0 & 0 & 0 & 0 & 0 & $-\overline{b_{11}}$ & $\overline{b_{11}}$ & $-b_{11}$ & $b_{11}$ \\
\hline
\end{tabular}


Table 13 Character table of $G^{X} \simeq 2 . A G L_{3}(2), X=A_{3}^{8}$

\begin{tabular}{|c|c|c|c|c|c|c|c|c|c|c|c|c|c|c|c|c|c|}
\hline$[g]$ & FS & $1 \mathrm{~A}$ & $2 A$ & $2 B$ & $4 \mathrm{~A}$ & 4B & $2 C$ & $3 A$ & $6 \mathrm{~A}$ & $6 B$ & $6 C$ & $8 \mathrm{~A}$ & $4 C$ & $7 \mathrm{~A}$ & $14 \mathrm{~A}$ & $7 \mathrm{~B}$ & $14 B$ \\
\hline$\left[g^{2}\right]$ & & $1 \mathrm{~A}$ & $1 \mathrm{~A}$ & $1 \mathrm{~A}$ & $2 A$ & $2 B$ & $1 \mathrm{~A}$ & $3 A$ & $3 A$ & $3 A$ & $3 \mathrm{~A}$ & $4 A$ & $2 C$ & $7 A$ & $7 \mathrm{~A}$ & $7 B$ & $7 \mathrm{~B}$ \\
\hline$\left[g^{3}\right]$ & & $1 \mathrm{~A}$ & $2 A$ & $2 B$ & $4 \mathrm{~A}$ & $4 B$ & $2 C$ & $1 \mathrm{~A}$ & $2 A$ & $2 B$ & $2 B$ & $8 \mathrm{~A}$ & $4 C$ & 7B & $14 \mathrm{~B}$ & $7 \mathrm{~A}$ & $14 \mathrm{~A}$ \\
\hline$\left[g^{7}\right]$ & & $1 \mathrm{~A}$ & $2 A$ & $2 B$ & $4 \mathrm{~A}$ & 4B & $2 C$ & $3 A$ & $6 \mathrm{~A}$ & $6 B$ & $6 C$ & $8 \mathrm{~A}$ & $4 C$ & $1 \mathrm{~A}$ & $2 A$ & $1 \mathrm{~A}$ & $2 A$ \\
\hline$\chi_{1}$ & + & 1 & 1 & 1 & 1 & 1 & 1 & 1 & 1 & 1 & 1 & 1 & 1 & & 1 & 1 & \\
\hline$\chi_{2}$ & $\circ$ & 3 & 3 & 3 & -1 & -1 & -1 & 0 & 0 & 0 & 0 & 1 & 1 & $b_{7}$ & $b_{7}$ & $\overline{b_{7}}$ & $\overline{b_{7}}$ \\
\hline$\chi_{3}$ & $\circ$ & 3 & 3 & 3 & -1 & -1 & -1 & 0 & 0 & 0 & 0 & 1 & 1 & $\overline{b_{7}}$ & $\overline{b_{7}}$ & $b_{7}$ & $b_{7}$ \\
\hline$\chi_{4}$ & + & 6 & 6 & 6 & 2 & 2 & 2 & 0 & 0 & 0 & 0 & 0 & 0 & -1 & -1 & -1 & -1 \\
\hline$\chi_{5}$ & + & 7 & 7 & 7 & -1 & -1 & -1 & 1 & 1 & 1 & 1 & -1 & -1 & 0 & 0 & 0 & \\
\hline$\chi_{6}$ & + & 8 & 8 & 8 & 0 & 0 & 0 & -1 & -1 & -1 & -1 & 0 & 0 & & 1 & 1 & \\
\hline$\chi_{7}$ & + & 7 & 7 & -1 & 3 & -1 & -1 & 1 & 1 & -1 & -1 & 1 & -1 & 0 & 0 & 0 & \\
\hline$\chi_{8}$ & + & 7 & 7 & -1 & -1 & -1 & 3 & 1 & 1 & -1 & -1 & -1 & 1 & & 0 & 0 & \\
\hline$\chi_{9}$ & + & 14 & 14 & -2 & 2 & -2 & 2 & -1 & -1 & 1 & 1 & 0 & 0 & & 0 & 0 & \\
\hline$\chi_{10}$ & + & 21 & 21 & -3 & 1 & 1 & -3 & 0 & 0 & 0 & 0 & -1 & 1 & 0 & 0 & 0 & \\
\hline$\chi_{11}$ & + & 21 & 21 & -3 & -3 & 1 & 1 & 0 & 0 & 0 & 0 & 1 & -1 & & 0 & 0 & \\
\hline$\chi_{12}$ & + & 8 & -8 & 0 & 0 & 0 & 0 & 2 & -2 & 0 & 0 & 0 & 0 & 1 & -1 & 1 & -1 \\
\hline$\chi_{13}$ & $\circ$ & 8 & -8 & 0 & 0 & 0 & 0 & -1 & 1 & $a_{3}$ & $\overline{a_{3}}$ & 0 & 0 & & -1 & 1 & -1 \\
\hline$\chi_{14}$ & $\circ$ & 8 & -8 & 0 & 0 & 0 & 0 & -1 & 1 & $\overline{a_{3}}$ & $a_{3}$ & 0 & 0 & 1 & -1 & 1 & -1 \\
\hline$\chi_{15}$ & $\circ$ & 24 & -24 & 0 & 0 & 0 & 0 & 0 & 0 & 0 & 0 & 0 & 0 & $\overline{b_{7}}$ & $-\overline{b_{7}}$ & $b_{7}$ & $-b_{7}$ \\
\hline$\chi_{16}$ & $\circ$ & 24 & -24 & 0 & 0 & 0 & 0 & 0 & 0 & 0 & 0 & 0 & 0 & $b_{7}$ & $-b_{7}$ & $\overline{b_{7}}$ & $-\overline{b_{7}}$ \\
\hline
\end{tabular}

Table 14 Character table of $G^{X} \simeq G L_{2}(5) / 2, X=A_{4}^{6}$

\begin{tabular}{rrrrrrrrrrrrrrrr}
\hline$[\boldsymbol{g}]$ & $\mathbf{F S}$ & $\mathbf{1 A}$ & $\mathbf{2 A}$ & $\mathbf{2 B}$ & $\mathbf{2 C}$ & $\mathbf{3 A}$ & $\mathbf{6 A}$ & $\mathbf{5 A}$ & $\mathbf{1 0 A}$ & $\mathbf{4 A}$ & $\mathbf{4 B}$ & $\mathbf{4 C}$ & $\mathbf{4 D}$ & $\mathbf{1 2 A}$ & $\mathbf{1 2 B}$ \\
\hline$\left[\boldsymbol{g}^{\mathbf{2}}\right]$ & & $\mathbf{1 A}$ & $\mathbf{1 A}$ & $\mathbf{1 A}$ & $\mathbf{1 A}$ & $\mathbf{3 A}$ & $\mathbf{3 A}$ & $\mathbf{5 A}$ & $\mathbf{5 A}$ & $\mathbf{2 A}$ & $\mathbf{2 A}$ & $\mathbf{2 C}$ & $\mathbf{2 C}$ & $\mathbf{6 A}$ & $\mathbf{6 A}$ \\
{$\left[\boldsymbol{g}^{3}\right]$} & & $\mathbf{1 A}$ & $\mathbf{2 A}$ & $\mathbf{2 B}$ & $\mathbf{2 C}$ & $\mathbf{1 A}$ & $\mathbf{2 A}$ & $\mathbf{5 A}$ & $\mathbf{1 0 A}$ & $\mathbf{4 B}$ & $\mathbf{4 A}$ & $\mathbf{4 D}$ & $\mathbf{4 C}$ & $\mathbf{4 B}$ & $\mathbf{4 A}$ \\
{$\left[\boldsymbol{g}^{\mathbf{5}}\right]$} & & $\mathbf{1 A}$ & $\mathbf{2 A}$ & $\mathbf{2 B}$ & $\mathbf{2 C}$ & $\mathbf{3 A}$ & $\mathbf{6 A}$ & $\mathbf{1 A}$ & $\mathbf{2 A}$ & $\mathbf{4 A}$ & $\mathbf{4 B}$ & $\mathbf{4 C}$ & $\mathbf{4 D}$ & $\mathbf{1 2 A}$ & $\mathbf{1 2 B}$ \\
\hline$\chi_{1}$ & + & 1 & 1 & 1 & 1 & 1 & 1 & 1 & 1 & 1 & 1 & 1 & 1 & 1 & 1 \\
$\chi_{2}$ & + & 1 & 1 & 1 & 1 & 1 & 1 & 1 & 1 & -1 & -1 & -1 & -1 & -1 & -1 \\
$\chi_{3}$ & + & 4 & 4 & 0 & 0 & 1 & 1 & -1 & -1 & 2 & 2 & 0 & 0 & -1 & -1 \\
$\chi_{4}$ & + & 4 & 4 & 0 & 0 & 1 & 1 & -1 & -1 & -2 & -2 & 0 & 0 & 1 & 1 \\
$\chi_{5}$ & + & 5 & 5 & 1 & 1 & -1 & -1 & 0 & 0 & 1 & 1 & -1 & -1 & 1 & 1 \\
$\chi_{6}$ & + & 5 & 5 & 1 & 1 & -1 & -1 & 0 & 0 & -1 & -1 & 1 & 1 & -1 & -1 \\
$\chi_{7}$ & + & 6 & 6 & -2 & -2 & 0 & 0 & 1 & 1 & 0 & 0 & 0 & 0 & 0 & 0 \\
$\chi_{8}$ & $\circ$ & 1 & -1 & 1 & -1 & 1 & -1 & 1 & -1 & $a_{1}$ & $-a_{1}$ & $a_{1}$ & $-a_{1}$ & $a_{1}$ & $-a_{1}$ \\
$\chi_{9}$ & 0 & 1 & -1 & 1 & -1 & 1 & -1 & 1 & -1 & $-a_{1}$ & $a_{1}$ & $-a_{1}$ & $a_{1}$ & $-a_{1}$ & $a_{1}$ \\
$\chi_{10}$ & 0 & 4 & -4 & 0 & 0 & 1 & -1 & -1 & 1 & $2 a_{1}$ & $-2 a_{1}$ & 0 & 0 & $-a_{1}$ & $a_{1}$ \\
$\chi_{11}$ & $\circ$ & 4 & -4 & 0 & 0 & 1 & -1 & -1 & 1 & $-2 a_{1}$ & $2 a_{1}$ & 0 & 0 & $a_{1}$ & $-a_{1}$ \\
$\chi_{12}$ & $\circ$ & 5 & -5 & 1 & -1 & -1 & 1 & 0 & 0 & $a_{1}$ & $-a_{1}$ & $-a_{1}$ & $a_{1}$ & $a_{1}$ & $-a_{1}$ \\
$\chi_{13}$ & $\circ$ & 5 & -5 & 1 & -1 & -1 & 1 & 0 & 0 & $-a_{1}$ & $a_{1}$ & $a_{1}$ & $-a_{1}$ & $-a_{1}$ & $a_{1}$ \\
$\chi_{14}$ & + & 6 & -6 & -2 & 2 & 0 & 0 & 1 & -1 & 0 & 0 & 0 & 0 & 0 & 0 \\
\hline
\end{tabular}

Table 15 Character table of $G^{X} \simeq G L_{2}(3), X \in\left\{A_{5}^{4} D_{4}, E_{6}^{4}\right\}$

\begin{tabular}{lrrrrrrrrr}
\hline$[\boldsymbol{g}]$ & $\mathbf{F S}$ & $\mathbf{1 A}$ & $\mathbf{2 A}$ & $\mathbf{2 B}$ & $\mathbf{4 A}$ & $\mathbf{3 A}$ & $\mathbf{6 A}$ & $\mathbf{8 A}$ & $\mathbf{8 B}$ \\
\hline$\left[\boldsymbol{g}^{\mathbf{2}}\right]$ & & $\mathbf{1 A}$ & $\mathbf{1 A}$ & $\mathbf{1 A}$ & $\mathbf{2 A}$ & $\mathbf{3 A}$ & $\mathbf{3 A}$ & $\mathbf{4 A}$ & $\mathbf{4 A}$ \\
{$\left[\boldsymbol{g}^{\mathbf{3}}\right]$} & & $\mathbf{1 A}$ & $\mathbf{2 A}$ & $\mathbf{2 B}$ & $\mathbf{4 A}$ & $\mathbf{1 A}$ & $\mathbf{2 A}$ & $\mathbf{8 A}$ & $\mathbf{8 B}$ \\
\hline$\chi_{1}$ & + & 1 & 1 & 1 & 1 & 1 & 1 & 1 & 1 \\
$\chi_{2}$ & + & 1 & 1 & -1 & 1 & 1 & 1 & -1 & -1 \\
$\chi_{3}$ & + & 2 & 2 & 0 & 2 & -1 & -1 & 0 & 0 \\
$\chi_{4}$ & + & 3 & 3 & -1 & -1 & 0 & 0 & 1 & 1 \\
$\chi_{5}$ & + & 3 & 3 & 1 & -1 & 0 & 0 & -1 & -1 \\
$\chi_{6}$ & 0 & 2 & -2 & 0 & 0 & -1 & 1 & $a_{2}$ & $\overline{a_{2}}$ \\
$\chi_{7}$ & 0 & 2 & -2 & 0 & 0 & -1 & 1 & $\overline{a_{2}}$ & $a_{2}$ \\
$\chi_{8}$ & + & 4 & -4 & 0 & 0 & 1 & -1 & 0 & 0 \\
\hline
\end{tabular}


Table 16 Character table of $G^{X} \simeq 3$. Sym $_{6}, X=D_{4}^{6}$

\begin{tabular}{rrrrrrrrrrrrrrrrrr}
\hline$[\boldsymbol{g}]$ & $\mathbf{F S}$ & $\mathbf{1 A}$ & $\mathbf{3 A}$ & $\mathbf{2 A}$ & $\mathbf{6 A}$ & $\mathbf{3 B}$ & $\mathbf{3 C}$ & $\mathbf{4 A}$ & $\mathbf{1 2 A}$ & $\mathbf{5 A}$ & $\mathbf{1 5 A}$ & $\mathbf{1 5 B}$ & $\mathbf{2 B}$ & $\mathbf{2 C}$ & $\mathbf{4 B}$ & $\mathbf{6 B}$ & $\mathbf{6 C}$ \\
\hline$\left[\boldsymbol{g}^{2}\right]$ & & $\mathbf{1 A}$ & $\mathbf{3 A}$ & $\mathbf{1 A}$ & $\mathbf{3 A}$ & $\mathbf{3 B}$ & $\mathbf{3 C}$ & $\mathbf{2 A}$ & $\mathbf{6 A}$ & $\mathbf{5 A}$ & $\mathbf{1 5 A}$ & $\mathbf{1 5 B}$ & $\mathbf{1 A}$ & $\mathbf{1 A}$ & $\mathbf{2 A}$ & $\mathbf{3 B}$ & $\mathbf{3 C}$ \\
{$\left[\boldsymbol{g}^{\mathbf{3}}\right]$} & & $\mathbf{1 A}$ & $\mathbf{1 A}$ & $\mathbf{2 A}$ & $\mathbf{2 A}$ & $\mathbf{1 A}$ & $\mathbf{1 A}$ & $\mathbf{4 A}$ & $\mathbf{4 A}$ & $\mathbf{5 A}$ & $\mathbf{5 A}$ & $\mathbf{5 A}$ & $\mathbf{2 B}$ & $\mathbf{2 C}$ & $\mathbf{4 B}$ & $\mathbf{2 B}$ & $\mathbf{2 C}$ \\
{$\left[\boldsymbol{g}^{5}\right]$} & & $\mathbf{1 A}$ & $\mathbf{3 A}$ & $\mathbf{2 A}$ & $\mathbf{6 A}$ & $\mathbf{3 B}$ & $\mathbf{3 C}$ & $\mathbf{4 A}$ & $\mathbf{1 2 A}$ & $\mathbf{1 A}$ & $\mathbf{3 A}$ & $\mathbf{3 A}$ & $\mathbf{2 B}$ & $\mathbf{2 C}$ & $\mathbf{4 B}$ & $\mathbf{6 B}$ & $\mathbf{6 C}$ \\
\hline$\chi_{1}$ & + & 1 & 1 & 1 & 1 & 1 & 1 & 1 & 1 & 1 & 1 & 1 & 1 & 1 & 1 & 1 & 1 \\
$\chi_{2}$ & + & 1 & 1 & 1 & 1 & 1 & 1 & 1 & 1 & 1 & 1 & 1 & -1 & -1 & -1 & -1 & -1 \\
$\chi_{3}$ & + & 5 & 5 & 1 & 1 & 2 & -1 & -1 & -1 & 0 & 0 & 0 & 3 & -1 & 1 & 0 & -1 \\
$\chi_{4}$ & + & 5 & 5 & 1 & 1 & 2 & -1 & -1 & -1 & 0 & 0 & 0 & -3 & 1 & -1 & 0 & 1 \\
$\chi_{5}$ & + & 5 & 5 & 1 & 1 & -1 & 2 & -1 & -1 & 0 & 0 & 0 & -1 & 3 & 1 & -1 & 0 \\
$\chi_{6}$ & + & 5 & 5 & 1 & 1 & -1 & 2 & -1 & -1 & 0 & 0 & 0 & 1 & -3 & -1 & 1 & 0 \\
$\chi_{7}$ & + & 16 & 16 & 0 & 0 & -2 & -2 & 0 & 0 & 1 & 1 & 1 & 0 & 0 & 0 & 0 & 0 \\
$\chi_{8}$ & + & 9 & 9 & 1 & 1 & 0 & 0 & 1 & 1 & -1 & -1 & -1 & 3 & 3 & -1 & 0 & 0 \\
$\chi_{9}$ & + & 9 & 9 & 1 & 1 & 0 & 0 & 1 & 1 & -1 & -1 & -1 & -3 & -3 & 1 & 0 & 0 \\
$\chi_{10}$ & + & 10 & 10 & -2 & -2 & 1 & 1 & 0 & 0 & 0 & 0 & 0 & 2 & -2 & 0 & -1 & 1 \\
$\chi_{11}$ & + & 10 & 10 & -2 & -2 & 1 & 1 & 0 & 0 & 0 & 0 & 0 & -2 & 2 & 0 & 1 & -1 \\
$\chi_{12}$ & 0 & 6 & -3 & -2 & 1 & 0 & 0 & 2 & -1 & 1 & $b_{15}$ & $b_{15}$ & 0 & 0 & 0 & 0 & 0 \\
$\chi_{13}$ & 0 & 6 & -3 & -2 & 1 & 0 & 0 & 2 & -1 & 1 & $b_{15}$ & $b_{15}$ & 0 & 0 & 0 & 0 & 0 \\
$\chi_{14}$ & + & 12 & -6 & 4 & -2 & 0 & 0 & 0 & 0 & 2 & -1 & -1 & 0 & 0 & 0 & 0 & 0 \\
$\chi_{15}$ & + & 18 & -9 & 2 & -1 & 0 & 0 & 2 & -1 & -2 & 1 & 1 & 0 & 0 & 0 & 0 & 0 \\
$\chi_{16}$ & + & 30 & -15 & -2 & 1 & 0 & 0 & -2 & 1 & 0 & 0 & 0 & 0 & 0 & 0 & 0 & 0 \\
\hline
\end{tabular}

Table 17 Character table of $G^{X} \simeq S L_{2}(3), X=A_{6}^{4}$

\begin{tabular}{|c|c|c|c|c|c|c|c|c|}
\hline$[g]$ & FS & $1 \mathrm{~A}$ & $2 A$ & $4 \mathrm{~A}$ & $3 A$ & $6 A$ & $3 B$ & $6 B$ \\
\hline$\left[g^{2}\right]$ & & $1 \mathrm{~A}$ & $1 \mathrm{~A}$ & $2 A$ & $3 B$ & $3 A$ & $3 A$ & $3 B$ \\
\hline$\left[g^{3}\right]$ & & $1 \mathrm{~A}$ & $2 A$ & $4 \mathrm{~A}$ & $1 \mathrm{~A}$ & $2 A$ & $1 \mathrm{~A}$ & $2 \mathrm{~A}$ \\
\hline$\chi_{1}$ & + & 1 & 1 & 1 & 1 & 1 & 1 & 1 \\
\hline$\chi_{2}$ & $\circ$ & 1 & 1 & 1 & $b_{3}$ & $\overline{b_{3}}$ & $\overline{b_{3}}$ & $b_{3}$ \\
\hline$\chi_{3}$ & $\circ$ & 1 & 1 & 1 & $\overline{b_{3}}$ & $b_{3}$ & $b_{3}$ & $\overline{b_{3}}$ \\
\hline$\chi_{4}$ & + & 3 & 3 & -1 & 0 & 0 & 0 & 0 \\
\hline$\chi_{5}$ & - & 2 & -2 & 0 & -1 & 1 & -1 & 1 \\
\hline$\chi_{6}$ & $\circ$ & 2 & -2 & 0 & $-\overline{b_{3}}$ & $b_{3}$ & $-b_{3}$ & $\overline{b_{3}}$ \\
\hline$\chi_{7}$ & $\circ$ & 2 & -2 & 0 & $-b_{3}$ & $\overline{b_{3}}$ & $-\overline{b_{3}}$ & $b_{3}$ \\
\hline
\end{tabular}

Table 18 Character table of $G^{X} \simeq D_{i h}, X=A_{7}^{2} D_{5}^{2}$

\begin{tabular}{lrrrrrr}
\hline$[\boldsymbol{g}]$ & $\mathbf{F S}$ & $\mathbf{1 A}$ & $\mathbf{2 A}$ & $\mathbf{2 B}$ & $\mathbf{2 C}$ & $\mathbf{4 A}$ \\
\hline$\left[\boldsymbol{g}^{2}\right]$ & & $\mathbf{1 A}$ & $\mathbf{1 A}$ & $\mathbf{1 A}$ & $\mathbf{1 A}$ & $\mathbf{2 A}$ \\
\hline$\chi_{1}$ & + & 1 & 1 & 1 & 1 & 1 \\
$\chi_{2}$ & + & 1 & 1 & -1 & -1 & 1 \\
$\chi_{3}$ & + & 1 & 1 & -1 & 1 & -1 \\
$\chi_{4}$ & + & 1 & 1 & 1 & -1 & -1 \\
$\chi_{5}$ & + & 2 & -2 & 0 & 0 & 0 \\
\hline
\end{tabular}

Table 19 Character table of $G^{X} \simeq D i h_{6}, X=A_{8}^{3}$

\begin{tabular}{lrrrrrrr}
\hline$[\boldsymbol{g}]$ & FS & $\mathbf{1 A}$ & $\mathbf{2 A}$ & $\mathbf{2 B}$ & $\mathbf{2 C}$ & $\mathbf{3 A}$ & $\mathbf{6 A}$ \\
\hline$\left[\boldsymbol{g}^{\mathbf{2}}\right]$ & & $\mathbf{1 A}$ & $\mathbf{1 A}$ & $\mathbf{1 A}$ & $\mathbf{1 A}$ & $\mathbf{3 A}$ & $\mathbf{3 A}$ \\
{$\left[\boldsymbol{g}^{3}\right]$} & & $\mathbf{1 A}$ & $\mathbf{2 A}$ & $\mathbf{2 B}$ & $\mathbf{2 C}$ & $\mathbf{1 A}$ & $\mathbf{2 A}$ \\
\hline$\chi_{1}$ & + & 1 & 1 & 1 & 1 & 1 & 1 \\
$\chi_{2}$ & + & 1 & 1 & -1 & -1 & 1 & 1 \\
$\chi_{3}$ & + & 2 & 2 & 0 & 0 & -1 & -1 \\
$\chi_{4}$ & + & 1 & -1 & -1 & 1 & 1 & -1 \\
$\chi_{5}$ & + & 1 & -1 & 1 & -1 & 1 & -1 \\
$\chi_{6}$ & + & 2 & -2 & 0 & 0 & -1 & 1 \\
\hline
\end{tabular}


Table 20 Character table of $G^{X} \simeq 4$, for $X \in\left\{A_{9}^{2} D_{6}, A_{12}^{2}\right\}$

\begin{tabular}{lrrrrr}
\hline$[\boldsymbol{g}]$ & $\mathbf{F S}$ & $\mathbf{1 A}$ & $\mathbf{2 A}$ & $\mathbf{4 A}$ & $\mathbf{4 B}$ \\
\hline$\left[\boldsymbol{g}^{2}\right]$ & $\mathbf{1 A}$ & $\mathbf{1 A}$ & $\mathbf{2 A}$ & $\mathbf{2 A}$ \\
\hline$\chi_{1}$ & 1 & 1 & 1 & 1 \\
$\chi_{2}$ & + & 1 & 1 & -1 & -1 \\
$\chi_{3}$ & + & 1 & -1 & $a_{1}$ & $\overline{a_{1}}$ \\
$\chi_{4}$ & 0 & 1 & -1 & $\overline{a_{1}}$ & $a_{1}$ \\
\hline
\end{tabular}

Table 21 Character table of $G^{X} \simeq P G L_{2}(3) \simeq S_{4 m_{4}}, X=D_{6}^{4}$

\begin{tabular}{lrrrrrr}
\hline$[\boldsymbol{g}]$ & FS & $\mathbf{1 A}$ & $\mathbf{2 A}$ & $\mathbf{3 A}$ & $\mathbf{2 B}$ & $\mathbf{4 A}$ \\
\hline$\left[\boldsymbol{g}^{2}\right]$ & & $\mathbf{1 A}$ & $\mathbf{1 A}$ & $\mathbf{3 A}$ & $\mathbf{1 A}$ & $\mathbf{2 A}$ \\
{$\left[\boldsymbol{g}^{\mathbf{3}}\right]$} & $\mathbf{1 A}$ & $\mathbf{2 A}$ & $\mathbf{1 A}$ & $\mathbf{2 B}$ & $\mathbf{4 A}$ \\
\hline$\chi_{1}$ & + & 1 & 1 & 1 & 1 & 1 \\
$\chi_{2}$ & + & 1 & 1 & 1 & -1 & -1 \\
$\chi_{3}$ & + & 2 & 2 & -1 & 0 & 0 \\
$\chi_{4}$ & + & 3 & -1 & 0 & 1 & -1 \\
$\chi_{5}$ & + & 3 & -1 & 0 & -1 & 1 \\
\hline
\end{tabular}

Table 22 Character table of $G^{X} \simeq 2$, for $X \in\left\{A_{11} D_{7} E_{6}, A_{15} D_{9}, A_{17} E_{7}, A_{24}, D_{10} E_{7}^{2}, D_{12}^{2}\right\}$

\begin{tabular}{llrr}
\hline$[g]$ & FS & $\mathbf{1 A}$ & $\mathbf{2 A}$ \\
\hline$\left[g^{2}\right]$ & & $\mathbf{1 A}$ & $\mathbf{1 A}$ \\
\hline$\chi_{1}$ & + & 1 & 1 \\
$\chi_{2}$ & + & 1 & -1 \\
\hline
\end{tabular}

Table 23 Character table of $G^{X} \simeq S_{3 m_{3}}, X \in\left\{D_{8}^{3}, E_{8}^{3}\right\}$

\begin{tabular}{lrrrr}
\hline$[g]$ & FS & $\mathbf{1 A}$ & $\mathbf{2 A}$ & $\mathbf{3 A}$ \\
\hline$\left[\boldsymbol{g}^{2}\right]$ & & $\mathbf{1 A}$ & $\mathbf{1 A}$ & $\mathbf{3 A}$ \\
{$\left[\boldsymbol{g}^{3}\right]$} & $\mathbf{1 A}$ & $\mathbf{2 A}$ & $\mathbf{1 A}$ \\
\hline$\chi_{1}$ & 1 & 1 & 1 \\
$\chi_{2}$ & + & 1 & -1 & 1 \\
$\chi_{3}$ & + & 2 & 0 & -1 \\
\hline
\end{tabular}


Table 24 Twisted Euler characters and Frame shapes at $\ell=2, X=A_{1}^{24}$

\begin{tabular}{|c|c|c|c|c|c|c|c|c|c|c|c|}
\hline [g] & $1 \mathrm{~A}$ & $2 A$ & $2 B$ & $3 \mathrm{~A}$ & 3B & $4 \mathrm{~A}$ & 4B & $4 C$ & $5 A$ & $6 \mathrm{~A}$ & $6 B$ \\
\hline$n_{g} \mid h_{g}$ & $1 \mid 1$ & $2 \mid 1$ & $2 \mid 2$ & $3 \mid 1$ & $3 \mid 3$ & $4 \mid 2$ & $4 \mid 1$ & $4 \mid 4$ & $5 \mid 1$ & $6 \mid 1$ & $6 \mid 6$ \\
\hline$\overline{\bar{\chi}}_{g}^{X_{A}}$ & 24 & 8 & 0 & 6 & 0 & 0 & 4 & 0 & 4 & 2 & 0 \\
\hline$\overline{\bar{\Pi}_{g}^{X_{A}}}$ & $1^{24}$ & $1^{8} 2^{8}$ & $2^{12}$ & $1^{6} 3^{6}$ & $3^{8}$ & $2^{4} 4^{4}$ & $1^{4} 2^{2} 4^{4}$ & $4^{6}$ & $1^{4} 5^{4}$ & $1^{2} 2^{2} 3^{2} 6^{2}$ & $\frac{6^{4}}{}$ \\
\hline$[g]$ & $7 A B$ & $8 \mathrm{~A}$ & $10 \mathrm{~A}$ & $11 \mathrm{~A}$ & $12 \mathrm{~A}$ & $12 B$ & $14 A B$ & $15 A B$ & $21 A B$ & $23 A B$ & \\
\hline$n_{g} \mid h_{g}$ & $7 \mid 1$ & $8 \mid 1$ & $10 \mid 2$ & $11 \mid 1$ & $12 \mid 2$ & $12 \mid 12$ & $14 \mid 1$ & $15 \mid 1$ & $21 \mid 3$ & $23 \mid 1$ & \\
\hline$\overline{\bar{\chi}}_{g}^{X_{A}}$ & 3 & 2 & 0 & 2 & 0 & 0 & 1 & 1 & 0 & 1 & \\
\hline$\overline{\bar{\Pi}_{g}^{X_{A}}}$ & $1^{3} 7^{3}$ & $1^{2} 2^{1} 4^{1} 8^{2}$ & $2^{2} 10^{2}$ & $1^{2} 11^{2}$ & $2^{1} 4^{1} 6^{1} 12^{1}$ & $12^{2}$ & $1^{1} 2^{1} 7^{1} 14^{1}$ & $1^{1} 3^{1} 5^{1} 15^{1}$ & $3^{1} 21^{1}$ & $1^{1} 23^{1}$ & \\
\hline
\end{tabular}


Table 25 Twisted Euler characters and Frame shapes at $\ell=3, X=A_{2}^{12}$

\begin{tabular}{|c|c|c|c|c|c|c|c|c|c|c|c|c|c|c|c|c|c|c|c|c|c|}
\hline [g] & $1 \mathrm{~A}$ & $2 A$ & $4 \mathrm{~A}$ & $2 B$ & $2 C$ & $3 A$ & $6 A$ & 3B & $6 B$ & 4B & $4 C$ & $5 A$ & $10 \mathrm{~A}$ & $12 \mathrm{~A}$ & $6 C$ & $6 \mathrm{D}$ & $8 A B$ & $8 C D$ & $20 A B$ & $11 \mathrm{AB}$ & $22 A B$ \\
\hline$n_{g} \mid h_{g}$ & $1 \mid 1$ & $1 \mid 4$ & $2 \mid 8$ & $2 \mid 1$ & $2 \mid 2$ & $3 \mid 1$ & $3 \mid 4$ & $3 \mid 3$ & $3 \mid 12$ & $4 \mid 2$ & $4 \mid 1$ & $5 \mid 1$ & $5 \mid 4$ & $6 \mid 24$ & $6 \mid 1$ & $6 \mid 2$ & $8 \mid 4$ & $8 \mid 1$ & $10 \mid 8$ & $11 \mid 1$ & $11 / 4$ \\
\hline $\bar{\chi}_{g}^{X_{A}}$ & 12 & 12 & 0 & 4 & 4 & 3 & 3 & 0 & 0 & 0 & 4 & 2 & 2 & 0 & 1 & 1 & 0 & 2 & 0 & 1 & 1 \\
\hline$\chi_{g}^{X_{A}}$ & 12 & -12 & 0 & 4 & -4 & 3 & -3 & 0 & 0 & 0 & 0 & 2 & -2 & 0 & 1 & -1 & 0 & 0 & 0 & 1 & -1 \\
\hline$\overline{\bar{\Pi}_{g}^{X_{A}}}$ & $1^{12}$ & $1^{12}$ & $2^{6}$ & $1^{4} 2^{4}$ & $1^{4} 2^{4}$ & $1^{3} 3^{3}$ & $1^{3} 3^{3}$ & $3^{4}$ & $3^{4}$ & $2^{2} 4^{2}$ & $1^{4} 4^{2}$ & $1^{2} 5^{2}$ & $1^{2} 5^{2}$ & $6^{2}$ & $1^{1} 2^{1} 3^{1} 6^{1}$ & $1^{1} 2^{1} 3^{1} 6^{1}$ & $4^{1} 8^{1}$ & $1^{2} 2^{1} 8^{1}$ & $2^{1} 10^{1}$ & $1^{1} 11^{1}$ & $1^{1} 11^{1}$ \\
\hline$\widetilde{\Pi}_{g}^{X_{A}}$ & $1^{24}$ & $2^{12}$ & $4^{6}$ & $1^{8} 2^{8}$ & $2^{12}$ & $1^{6} 3^{6}$ & $2^{3} 6^{3}$ & $3^{8}$ & $6^{4}$ & $2^{4} 4^{4}$ & $1^{4} 2^{2} 4^{4}$ & $1^{4} 5^{4}$ & $2^{2} 10^{2}$ & $12^{2}$ & $1^{2} 2^{2} 3^{2} 6^{2}$ & $2^{2} 6^{2}$ & $4^{2} 8^{2}$ & $1^{2} 2^{1} 4^{1} 8^{2}$ & $4^{1} 20^{1}$ & $1^{2} 11^{2}$ & $2^{1} 22^{1}$ \\
\hline
\end{tabular}


Table 26 Twisted Euler characters and Frame shapes at $\ell=4, X=A_{3}^{8}$

\begin{tabular}{lrrrrrrrrrrrrr}
\hline$[\boldsymbol{g}]$ & $\mathbf{1 A}$ & $\mathbf{2 A}$ & $\mathbf{2 B}$ & $\mathbf{4 A}$ & $\mathbf{4 B}$ & $\mathbf{2 C}$ & $\mathbf{3 A}$ & $\mathbf{6 A}$ & $\mathbf{6 B C}$ & $\mathbf{8 A}$ & $\mathbf{4 C}$ & $\mathbf{7 A B}$ & $\mathbf{1 4 A B}$ \\
\hline $\boldsymbol{n}_{\boldsymbol{g}} \mid \boldsymbol{h}_{\boldsymbol{g}}$ & $\mathbf{1 | 1}$ & $\mathbf{1} \mid \mathbf{2}$ & $\mathbf{2 | 2}$ & $\mathbf{2} \mid \mathbf{4}$ & $\mathbf{4} \mid \mathbf{4}$ & $\mathbf{2 | 1}$ & $\mathbf{3} \mid \mathbf{1}$ & $\mathbf{3} \mid \mathbf{2}$ & $\mathbf{6 | 2}$ & $\mathbf{4} \mid \mathbf{8}$ & $\mathbf{4 | 1}$ & $\mathbf{7 | 1}$ & $\mathbf{7 | 2}$ \\
\hline $\bar{\chi}_{g}^{X_{A}}$ & 8 & 8 & 0 & 0 & 0 & 4 & 2 & 2 & 0 & 0 & 2 & 1 & 1 \\
$\chi_{g}^{X_{A}}$ & 8 & -8 & 0 & 0 & 0 & 0 & 2 & -2 & 0 & 0 & 0 & 1 & -1 \\
\hline $\bar{\Pi}_{g}^{X_{A}}$ & $1^{8}$ & $1^{8}$ & $2^{4}$ & $2^{4}$ & $4^{2}$ & $1^{4} 2^{2}$ & $1^{2} 3^{2}$ & $1^{2} 3^{2}$ & $2^{1} 6^{1}$ & $4^{2}$ & $1^{2} 2^{1} 4^{1}$ & $1^{1} 7^{1}$ & $1^{1} 7^{1}$ \\
$\widetilde{\Pi}_{g}^{X_{A}}$ & $1^{24}$ & $1^{8} 2^{8}$ & $2^{12}$ & $2^{4} 4^{4}$ & $4^{6}$ & $1^{8} 2^{8}$ & $1^{6} 3^{6}$ & $1^{2} 2^{2} 3^{2} 6^{2}$ & $2^{3} 6^{3}$ & $4^{2} 8^{2}$ & $1^{4} 2^{2} 4^{4}$ & $1^{3} 7^{3}$ & $1^{1} 2^{1} 7^{1} 14^{1}$ \\
\hline
\end{tabular}

Table 27 Twisted Euler characters and Frame shapes at $\ell=5, X=A_{4}^{6}$

\begin{tabular}{lrrrrrrrrrrr}
\hline$[\boldsymbol{g}]$ & $\mathbf{1 A}$ & $\mathbf{2 A}$ & $\mathbf{2 B}$ & $\mathbf{2 C}$ & $\mathbf{3 A}$ & $\mathbf{6 A}$ & $\mathbf{5 A}$ & $\mathbf{1 0 A}$ & $\mathbf{4 A B}$ & $\mathbf{4 C D}$ & $\mathbf{1 2 A B}$ \\
\hline $\boldsymbol{n}_{\boldsymbol{g}} \mid \boldsymbol{h}_{\boldsymbol{g}}$ & $\mathbf{1 | 1}$ & $\mathbf{1 | 4}$ & $\mathbf{2 | 2}$ & $\mathbf{2} \mid \mathbf{1}$ & $\mathbf{3 | 3}$ & $\mathbf{3 | 1 2}$ & $\mathbf{5 | 1}$ & $\mathbf{5 | 4}$ & $\mathbf{2} \mid \mathbf{8}$ & $\mathbf{4} \mid \mathbf{1}$ & $\mathbf{6 | 2 4}$ \\
\hline $\bar{\chi}_{g}^{X_{A}}$ & 6 & 6 & 2 & 2 & 0 & 0 & 1 & 1 & 0 & 2 & 0 \\
$\chi_{g}^{X_{A}}$ & 6 & -6 & -2 & 2 & 0 & 0 & 1 & -1 & 0 & 0 & 0 \\
\hline $\bar{\Pi}_{g}^{X_{A}}$ & $1^{6}$ & $1^{6}$ & $1^{2} 2^{2}$ & $1^{2} 2^{2}$ & $3^{2}$ & $3^{2}$ & $1^{1} 5^{1}$ & $1^{1} 5^{1}$ & $2^{3}$ & $1^{2} 4^{1}$ & $6^{1}$ \\
$\widetilde{\Pi}_{g}^{X_{A}}$ & $1^{24}$ & $2^{12}$ & $2^{12}$ & $1^{8} 2^{8}$ & $3^{8}$ & $6^{4}$ & $1^{4} 5^{4}$ & $2^{2} 10^{2}$ & $4^{6}$ & $1^{4} 2^{2} 4^{4}$ & $12^{2}$ \\
\hline
\end{tabular}

Table 28 Twisted Euler characters and Frame shapes at $\ell=6, X=A_{5}^{4} D_{4}$

\begin{tabular}{lrrrrrrr}
\hline$[\boldsymbol{g}]$ & $\mathbf{1 A}$ & $\mathbf{2 A}$ & $\mathbf{2 B}$ & $\mathbf{4 A}$ & $\mathbf{3 A}$ & $\mathbf{6 A}$ & $\mathbf{8 A \mathbf { B }}$ \\
\hline $\boldsymbol{n}_{\boldsymbol{g}} \mid \boldsymbol{h}_{\boldsymbol{g}}$ & $\mathbf{1} \mid \mathbf{1}$ & $\mathbf{1 | 2}$ & $\mathbf{2} \mid \mathbf{1}$ & $\mathbf{2} \mid \mathbf{2}$ & $\mathbf{3} \mid \mathbf{1}$ & $\mathbf{3} \mid \mathbf{2}$ & $\mathbf{4} \mathbf{2}$ \\
\hline $\bar{\chi}_{g}^{X_{A}}$ & 4 & 4 & 2 & 0 & 1 & 1 & 0 \\
$\chi_{g}^{X_{A}}$ & 4 & -4 & 0 & 0 & 1 & -1 & 0 \\
\hline $\bar{\Pi}_{g}^{X_{A}}$ & $1^{4}$ & $1^{4}$ & $1^{2} 2^{1}$ & $2^{2}$ & $1^{1} 3^{1}$ & $1^{1} 3^{1}$ & $4^{1}$ \\
$\widetilde{\Pi}_{g}^{X_{A}}$ & $1^{20}$ & $1^{4} 2^{8}$ & $1^{6} 2^{7}$ & $2^{2} 4^{4}$ & $1^{5} 3^{5}$ & $1^{1} 2^{2} 3^{1} 6^{2}$ & $4^{1} 8^{2}$ \\
\hline $\bar{\chi}_{g}^{X_{D}}$ & 1 & 1 & 1 & 1 & 1 & 1 & 1 \\
$\chi_{g}^{X_{D}}$ & 1 & 1 & -1 & 1 & 1 & 1 & -1 \\
$\check{\chi}_{g}^{X_{D}}$ & 2 & 2 & 0 & 2 & -1 & -1 & 0 \\
\hline $\bar{\Pi}_{g}^{X_{D}}$ & $1^{1}$ & $1^{1}$ & $1^{1}$ & $1^{1}$ & $1^{1}$ & $1^{1}$ & $1^{1}$ \\
$\widetilde{\Pi}_{g}^{X_{D}}$ & $1^{4}$ & $1^{4}$ & $1^{2} 2^{1}$ & $1^{4}$ & $1^{1} 3^{1}$ & $1^{1} 3^{1}$ & $1^{2} 2^{1}$ \\
$\widetilde{\Pi}_{g}^{X}$ & $1^{24}$ & $1^{8} 2^{8}$ & $1^{8} 2^{8}$ & $1^{4} 2^{2} 4^{4}$ & $1^{6} 3^{6}$ & $1^{2} 2^{2} 3^{2} 6^{2}$ & $1^{2} 2^{1} 4^{1} 8^{2}$ \\
\hline & & & & & & &
\end{tabular}


Table 29 Twisted Euler characters and Frame shapes at $\ell=6+3, X=D_{4}^{6}$

\begin{tabular}{|c|c|c|c|c|c|c|c|c|c|c|c|c|c|c|c|}
\hline [g] & $1 \mathrm{~A}$ & $3 A$ & $2 A$ & $6 \mathrm{~A}$ & $3 B$ & $6 C$ & $4 A$ & $12 \mathrm{~A}$ & $5 A$ & $15 \mathrm{AB}$ & $2 B$ & $2 \mathrm{C}$ & 4B & $6 B$ & $6 C$ \\
\hline$\overline{n_{g} \mid h_{g}}$ & $1 \mid 1$ & $1 \mid 3$ & $2 \mid 1$ & $2 \mid 3$ & $3 \mid 1$ & $3 \mid 3$ & $4 \mid 2$ & $4 \mid 6$ & $5 \mid 1$ & $5 \mid 3$ & $2 \mid 1$ & $2 \mid 2$ & $4 \mid 1$ & $6 \mid 1$ & $6 \mid 6$ \\
\hline $\bar{\chi}_{g}^{X_{D}}$ & 6 & 6 & 2 & 2 & 3 & 0 & 0 & 0 & 1 & 1 & 4 & 0 & 2 & 1 & 0 \\
\hline$\chi_{g}^{X_{D}}$ & 6 & 6 & 2 & 2 & 3 & 0 & 0 & 0 & 1 & 1 & -4 & 0 & -2 & -1 & 0 \\
\hline$\check{\chi}_{g}^{X_{D}}$ & 12 & -6 & 4 & -2 & 0 & 0 & 0 & 0 & 2 & -1 & 0 & 0 & 0 & 0 & 0 \\
\hline$\overline{\bar{\Pi}_{g}^{X_{D}}}$ & $1^{6}$ & $1^{6}$ & $1^{2} 2^{2}$ & $1^{2} 2^{2}$ & $1^{3} 3^{1}$ & $3^{2}$ & $2^{1} 4^{1}$ & $2^{1} 4^{1}$ & $1^{1} 5^{1}$ & $1^{1} 5^{1}$ & $1^{4} 2^{1}$ & $2^{3}$ & $1^{2} 4^{1}$ & $1^{1} 2^{1} 3^{1}$ & $\overline{6^{1}}$ \\
\hline$\widetilde{\Pi}_{g}^{x_{D}}$ & $1^{24}$ & $1^{6} 3^{6}$ & $1^{8} 2^{8}$ & $1^{2} 2^{2} 3^{2} 6^{2}$ & $1^{6} 3^{6}$ & $3^{8}$ & $2^{4} 4^{4}$ & $2^{1} 4^{1} 6^{1} 12^{1}$ & $1^{4} 5^{4}$ & $1^{1} 3^{1} 5^{1} 15^{1}$ & $1^{2} 2^{8}$ & $2^{12}$ & $1^{4} 2^{2} 4^{4}$ & $1^{2} 2^{2} 3^{2} 6^{2}$ & $6^{4}$ \\
\hline
\end{tabular}


Table 30 Twisted Euler characters and Frame shapes at $\ell=7, X=A_{6}^{4}$

\begin{tabular}{lrrrrr}
\hline$[\boldsymbol{g}]$ & $\mathbf{1 A}$ & $\mathbf{2 A}$ & $\mathbf{4 A}$ & $\mathbf{3 A B}$ & $\mathbf{6 A B}$ \\
\hline $\boldsymbol{n}_{\boldsymbol{g}} \mid \boldsymbol{h}_{\boldsymbol{g}}$ & $\mathbf{1 | 1}$ & $\mathbf{1 | 4}$ & $\mathbf{2 | 8}$ & $\mathbf{3} \mid \mathbf{1}$ & $\mathbf{3 | \mathbf { 4 }}$ \\
\hline $\bar{\chi}_{g}^{X_{A}}$ & 4 & 4 & 0 & 1 & 1 \\
$\chi_{g}^{X_{A}}$ & 4 & -4 & 0 & 1 & -1 \\
\hline $\bar{\Pi}_{g}^{X_{A}}$ & $1^{4}$ & $1^{4}$ & $2^{2}$ & $1^{1} 3^{1}$ & $1^{1} 3^{1}$ \\
$\widetilde{\Pi}_{g}^{X_{A}}$ & $1^{24}$ & $2^{12}$ & $4^{6}$ & $1^{6} 3^{6}$ & $2^{3} 6^{3}$ \\
\hline
\end{tabular}

Table 31 Twisted Euler characters and Frame shapes at $\ell=8, X=A_{7}^{2} D_{5}^{2}$

\begin{tabular}{lrrrrr}
\hline$[\boldsymbol{g}]$ & $\mathbf{1 A}$ & $\mathbf{2 A}$ & $\mathbf{2 B}$ & $\mathbf{2 C}$ & $\mathbf{4 A}$ \\
\hline $\boldsymbol{n}_{\boldsymbol{g}} \mid \boldsymbol{h}_{\boldsymbol{g}}$ & $\mathbf{1 | 1}$ & $\mathbf{1 | 2}$ & $\mathbf{2} \mid \mathbf{1}$ & $\mathbf{2 | 1}$ & $\mathbf{2 | \mathbf { 4 }}$ \\
\hline $\bar{\chi}_{g}^{X_{A}}$ & 2 & 2 & 0 & 2 & 0 \\
$\chi_{g}^{X_{A}}$ & 2 & -2 & 0 & 0 & 0 \\
\hline $\bar{\Pi}_{g}^{X_{A}}$ & $1^{2}$ & $1^{2}$ & $2^{1}$ & $2^{2}$ & $2^{1}$ \\
$\widetilde{\Pi}_{g}^{X_{A}}$ & $1^{14}$ & $1^{2} 2^{6}$ & 2 & $1^{8} 2^{3}$ & $2^{1} 4^{3}$ \\
\hline $\bar{\chi}_{g}^{X_{D}}$ & 2 & 2 & 0 & 0 & 0 \\
$\chi_{g}^{X_{D}}$ & 2 & -2 & $2^{2}$ & 0 & 0 \\
\hline $\bar{\Pi}_{g}^{X_{D}}$ & $1^{2}$ & $1^{2}$ & $1^{8} 2^{1}$ & $2^{1}$ & $2^{1}$ \\
$\widetilde{\Pi}_{g}^{X_{D}}$ & $1^{10}$ & $1^{6} 2^{2}$ & $1^{8} 2^{8}$ & $2^{5}$ & $2^{3} 4^{1}$ \\
$\widetilde{\Pi}_{g}^{X}$ & $1^{24}$ & $1^{8} 2^{8}$ & & & $2^{4} 4^{4}$ \\
\hline
\end{tabular}

Table 32 Twisted Euler characters and Frame shapes at $\ell=9, X=A_{8}^{3}$

\begin{tabular}{lrrrrrr}
\hline$[\boldsymbol{g}]$ & $\mathbf{1 A}$ & $\mathbf{2 A}$ & $\mathbf{2 B}$ & $\mathbf{2 C}$ & $\mathbf{3 A}$ & $\mathbf{6 A}$ \\
\hline $\boldsymbol{n}_{\boldsymbol{g}} \mid \boldsymbol{h}_{\boldsymbol{g}}$ & $\mathbf{1 | 1}$ & $\mathbf{1 | 4}$ & $\mathbf{2} \mid \mathbf{1}$ & $\mathbf{2 | 2}$ & $\mathbf{3} \mid \mathbf{3}$ & $\mathbf{3} \mid \mathbf{1 2}$ \\
\hline $\bar{\chi}_{g}^{X_{A}}$ & 3 & 3 & 1 & 1 & 0 & 0 \\
$\chi_{g}^{X_{A}}$ & 3 & -3 & 1 & -1 & 0 & 0 \\
\hline $\bar{\Pi}_{g}^{X_{A}}$ & $1^{3}$ & $1^{3}$ & $1^{1} 2^{1}$ & $1^{1} 2^{1}$ & $3^{1}$ & $3^{1}$ \\
$\widetilde{\Pi}_{g}^{X_{A}}$ & $1^{24}$ & $2^{12}$ & $1^{8} 2^{8}$ & $2^{12}$ & $3^{8}$ & $6^{4}$ \\
\hline
\end{tabular}

Table 33 Twisted Euler characters and Frame shapes at $\ell=10, X=A_{9}^{2} D_{6}$

\begin{tabular}{lrrr}
\hline$[\boldsymbol{g}]$ & $\mathbf{1 A}$ & $\mathbf{2 A}$ & $\mathbf{4 A B}$ \\
\hline $\boldsymbol{n}_{\boldsymbol{g}} \mid \boldsymbol{h}_{\boldsymbol{g}}$ & $\mathbf{1 | 1}$ & $\mathbf{1 | 2}$ & $\mathbf{2 | \mathbf { 2 }}$ \\
\hline $\bar{\chi}_{g}^{X_{A}}$ & 2 & 2 & 0 \\
$\chi_{g}^{X_{A}}$ & 2 & -2 & 0 \\
\hline $\bar{\Pi}_{g}^{X_{A}}$ & $1^{2}$ & $1^{2}$ & $2^{1}$ \\
$\widetilde{\Pi}_{g}^{X_{A}}$ & $1^{18}$ & $1^{2} 2^{8}$ & $2^{1} 4^{4}$ \\
\hline $\bar{\chi}_{g}^{X_{D}}$ & 1 & 1 & 1 \\
$\chi_{g}^{X_{D}}$ & 1 & 1 & -1 \\
\hline $\bar{\Pi}_{g}^{X_{D}}$ & $1^{1}$ & $1^{1}$ & $1^{1}$ \\
$\widetilde{\Pi}_{g}^{X_{D}}$ & $1^{6}$ & $1^{6}$ & $1^{4} 2^{1}$ \\
$\widetilde{\Pi}_{g}^{X}$ & $1^{24}$ & $1^{8} 2^{8}$ & $1^{4} 2^{2} 4^{4}$ \\
\hline & & &
\end{tabular}

Table 34 Twisted Euler characters and Frame shapes at $\ell=10+5, X=D_{6}^{4}$

\begin{tabular}{lrrrrr}
\hline$[\boldsymbol{g}]$ & $\mathbf{1 A}$ & $\mathbf{2 A}$ & $\mathbf{3 A}$ & $\mathbf{2 B}$ & $\mathbf{4 A}$ \\
\hline $\boldsymbol{n}_{\boldsymbol{g}} \boldsymbol{h}_{\boldsymbol{g}}$ & $\mathbf{1 | 1}$ & $\mathbf{2} \mid \mathbf{2}$ & $\mathbf{3} \mid \mathbf{1}$ & $\mathbf{2} \mid \mathbf{1}$ & $\mathbf{4 | \mathbf { 4 }}$ \\
\hline $\bar{\chi}_{g}^{X_{D}}$ & 4 & 0 & 1 & 2 & 0 \\
$\chi_{g}^{X_{D}}$ & 4 & 0 & 1 & -2 & 0 \\
\hline $\bar{\Pi}_{g}^{X_{D}}$ & $1^{4}$ & $2^{2}$ & $1^{1} 3^{1}$ & $1^{2} 2^{1}$ & $4^{1}$ \\
$\widetilde{\Pi}_{g}^{X_{D}}$ & $1^{24}$ & $2^{12}$ & $1^{6} 3^{6}$ & $1^{8} 2^{8}$ & $4^{6}$ \\
\hline
\end{tabular}


Table 35 Twisted Euler characters and Frame shapes at $\ell=12, X=A_{11} D_{7} E_{6}$

\begin{tabular}{lrr}
\hline$[\boldsymbol{g}]$ & $\mathbf{1 A}$ & $\mathbf{2 A}$ \\
\hline $\boldsymbol{n}_{\boldsymbol{g}} \mid \boldsymbol{h}_{\boldsymbol{g}}$ & $\mathbf{1 | 1}$ & $\mathbf{1 | \mathbf { 2 }}$ \\
\hline $\bar{\chi}_{g}^{X_{A}}$ & 1 & 1 \\
$\chi_{g}^{X_{A}}$ & 1 & -1 \\
\hline $\bar{\Pi}_{g}^{X_{A}}$ & $1^{1}$ & $1^{1}$ \\
$\widetilde{\Pi}_{g}^{X_{A}}$ & $1^{11}$ & $1^{1} 2^{5}$ \\
\hline $\bar{\chi}_{g}^{X_{D}}$ & 1 & 1 \\
$\chi_{g}^{X_{D}}$ & 1 & -1 \\
\hline $\bar{\Pi}_{g}^{X_{D}}$ & $1^{1}$ & $1^{1}$ \\
$\widetilde{\Pi}_{g}^{X_{D}}$ & $1^{7}$ & $1^{5} 2^{1}$ \\
\hline $\bar{\chi}_{g}^{X_{E}}$ & 1 & 1 \\
$\chi_{g}^{X_{E}}$ & 1 & -1 \\
\hline $\bar{\Pi}_{g}^{X_{E}}$ & 1 & $1^{1}$ \\
$\widetilde{\Pi}_{g}^{X_{E}}$ & $1^{1}$ & $1^{2} 2^{2}$ \\
$\widetilde{\Pi}_{g}^{X}$ & $1^{6}$ & $1^{8} 2^{8}$ \\
\hline & $1^{24}$ & \\
\hline
\end{tabular}

Table 36 Twisted Euler characters and Frame shapes at $\ell=12+4, X=E_{6}^{4}$

\begin{tabular}{lrrrrrrr}
\hline$[\boldsymbol{g}]$ & $\mathbf{1 A}$ & $\mathbf{2 A}$ & $\mathbf{2 B}$ & $\mathbf{4 A}$ & $\mathbf{3 A}$ & $\mathbf{6 A}$ & $\mathbf{8 A B}$ \\
\hline $\boldsymbol{n}_{\boldsymbol{g}} \mid \boldsymbol{h}_{\boldsymbol{g}}$ & $\mathbf{1 | 1}$ & $\mathbf{1 | 2}$ & $\mathbf{2} \mid \mathbf{1}$ & $\mathbf{2 | 4}$ & $\mathbf{3} \mid \mathbf{1}$ & $\mathbf{3 | 2}$ & $\mathbf{4} \mid \mathbf{8}$ \\
\hline $\bar{\chi}_{g}^{X_{E}}$ & 4 & 4 & 2 & 0 & 1 & 1 & 0 \\
$\chi_{g}^{X_{E}}$ & 4 & -4 & 0 & 0 & 1 & -1 & 0 \\
\hline $\bar{\Pi}_{g}^{X_{E}}$ & $1^{4}$ & $1^{4}$ & $1^{2} 2^{1}$ & $2^{2}$ & $1^{1} 3^{1}$ & $1^{1} 3^{1}$ & $4^{1}$ \\
$\widetilde{\Pi}_{g}^{X_{E}}$ & $1^{24}$ & $1^{8} 2^{8}$ & $1^{8} 2^{8}$ & $2^{4} 4^{4}$ & $1^{6} 3^{6}$ & $1^{2} 2^{2} 3^{2} 6^{2}$ & $4^{2} 8^{2}$ \\
\hline
\end{tabular}

Table 37 Twisted Euler characters and Frame shapes at $\ell=13, X=A_{12}^{2}$

\begin{tabular}{lrrr}
\hline$[\boldsymbol{g}]$ & $\mathbf{1 A}$ & $\mathbf{2 A}$ & $\mathbf{4 A B}$ \\
\hline $\boldsymbol{n}_{\boldsymbol{g}} \mid \boldsymbol{h}_{\boldsymbol{g}}$ & $\mathbf{1 | 1}$ & $\mathbf{1 | 4}$ & $\mathbf{2 | \boldsymbol { 8 }}$ \\
\hline $\bar{\chi}_{g}^{X_{A}}$ & 2 & 2 & 0 \\
$\chi_{g}^{X_{A}}$ & 2 & -2 & 0 \\
\hline $\bar{\Pi}_{g}^{X_{A}}$ & $1^{2}$ & $1^{2}$ & $2^{1}$ \\
$\widetilde{\Pi}_{g}^{X_{A}}$ & $1^{24}$ & $2^{12}$ & $4^{6}$ \\
\hline
\end{tabular}


Table 38 Twisted Euler characters and Frame shapes at $\ell=14+7, X=D_{8}^{3}$

\begin{tabular}{lrrr}
\hline$[\boldsymbol{g}]$ & $\mathbf{1 A}$ & $\mathbf{2 A}$ & $\mathbf{3 A}$ \\
\hline $\boldsymbol{n}_{\boldsymbol{g}} \mid \boldsymbol{h}_{\boldsymbol{g}}$ & $\mathbf{1 | 1}$ & $\mathbf{2} \mid \mathbf{1}$ & $\mathbf{3 | 3}$ \\
\hline $\bar{\chi}_{g}^{X_{D}}$ & 3 & 1 & 0 \\
$\chi_{g}^{X_{D}}$ & 3 & 1 & 0 \\
\hline $\bar{\Pi}_{g}^{X_{D}}$ & $1^{3}$ & $1^{1} 2^{1}$ & $3^{1}$ \\
$\widetilde{\Pi}_{g}^{X_{D}}$ & $1^{24}$ & $1^{8} 2^{8}$ & $3^{8}$ \\
\hline
\end{tabular}

Table 39 Twisted Euler characters and Frame shapes at $\ell=16, X=A_{15} D_{9}$

\begin{tabular}{lrr}
\hline $\boldsymbol{g}]$ & $\mathbf{1 A}$ & $\mathbf{2 A}$ \\
\hline $\boldsymbol{n}_{\boldsymbol{g}} \mid \boldsymbol{h}_{\boldsymbol{g}}$ & $\mathbf{1 | 1}$ & $\mathbf{1 | \mathbf { 2 }}$ \\
\hline $\bar{\chi}_{g}^{X_{A}}$ & 1 & 1 \\
$\chi_{g}^{X_{A}}$ & 1 & -1 \\
\hline $\bar{\Pi}_{g}^{X_{A}}$ & $1^{1}$ & $1^{1}$ \\
$\widetilde{\Pi}_{g}^{X_{A}}$ & $1^{15}$ & $1^{1} 2^{7}$ \\
\hline $\bar{\chi}_{g}^{X_{D}}$ & 1 & 1 \\
$\chi_{g}^{X_{D}}$ & 1 & -1 \\
\hline $\bar{\Pi}_{g}^{X_{D}}$ & $1^{1}$ & $1^{1}$ \\
$\widetilde{\Pi}_{g}^{X_{D}}$ & $1^{9}$ & $1^{7} 2^{1}$ \\
\hline$\widetilde{\Pi}_{g}^{X}$ & $1^{24}$ & $1^{8} 2^{8}$ \\
\hline
\end{tabular}

Table 40 Twisted Euler characters and Frame shapes at $\ll=18, X=A_{17} E_{7}$

\begin{tabular}{lrr}
\hline $\boldsymbol{g}]$ & $\mathbf{1 A}$ & $\mathbf{2 A}$ \\
\hline $\boldsymbol{n}_{\boldsymbol{g}} \mid \boldsymbol{h}_{\boldsymbol{g}}$ & $\mathbf{1 | 1}$ & $\mathbf{1 | 2}$ \\
\hline $\bar{\chi}_{g}^{X_{A}}$ & 1 & 1 \\
$\chi_{g}^{X_{A}}$ & 1 & -1 \\
\hline $\bar{\Pi}_{g}^{X_{A}}$ & $1^{1}$ & $1^{1}$ \\
$\widetilde{\Pi}_{g}^{X_{A}}$ & $1^{17}$ & $1^{1} 2^{8}$ \\
\hline $\bar{\chi}_{g}^{X_{E}}$ & 1 & 1 \\
\hline $\bar{\Pi}_{g}^{X_{E}}$ & $1^{1}$ & $1^{1}$ \\
$\widetilde{\Pi}_{g}^{X_{E}}$ & $1^{7}$ & $1^{7}$ \\
$\widetilde{\Pi}_{g}^{X}$ & $1^{24}$ & $1^{8} 2^{8}$ \\
\hline
\end{tabular}

Table 41 Twisted Euler characters and Frame shapes at $\ll=18+9, X=D_{10} E_{7}^{2}$

\begin{tabular}{lrr}
\hline$[\boldsymbol{g}]$ & $\mathbf{1 A}$ & $\mathbf{2 A}$ \\
\hline $\boldsymbol{n}_{\boldsymbol{g}} \mid \boldsymbol{h}_{\boldsymbol{g}}$ & $\mathbf{1 | 1}$ & $\mathbf{2 | 1}$ \\
\hline $\bar{\chi}_{g}^{X_{D}}$ & 1 & 1 \\
$\chi_{g}^{X_{D}}$ & 1 & -1 \\
\hline $\bar{\Pi}_{g}^{X_{D}}$ & $1^{1}$ & $1^{1}$ \\
$\widetilde{\Pi}_{g}^{X_{D}}$ & $1^{10}$ & $1^{8} 2^{1}$ \\
\hline $\bar{\chi}_{g}^{X_{E}}$ & 2 & 0 \\
\hline $\bar{\Pi}_{g}^{X_{E}}$ & $1^{2}$ & $2^{1}$ \\
$\widetilde{\Pi}_{g}^{X_{E}}$ & $1^{14}$ & $2^{7}$ \\
$\widetilde{\Pi}_{g}^{X}$ & $1^{24}$ & $1^{8} 2^{8}$ \\
\hline
\end{tabular}


Table 42 Twisted Euler characters and Frame shapes at $\ll=22+11, X=D_{12}^{2}$

\begin{tabular}{lrr}
\hline $\boldsymbol{g}]$ & $\mathbf{1 A}$ & $\mathbf{2 A}$ \\
\hline $\boldsymbol{n}_{\boldsymbol{g}} \mid \boldsymbol{h}_{\boldsymbol{g}}$ & $\mathbf{1 | 1}$ & $\mathbf{2 | 2}$ \\
\hline $\bar{\chi}_{g}^{X_{D}}$ & 2 & 0 \\
$\chi_{g}^{X_{D}}$ & 2 & 0 \\
\hline $\bar{\Pi}_{g}^{X_{D}}$ & $1^{2}$ & $2^{1}$ \\
$\widetilde{\Pi}_{g}^{X_{D}}$ & $1^{24}$ & $2^{12}$ \\
\hline
\end{tabular}

Table 43 Twisted Euler characters and Frame shapes at $\ll=25, X=A_{24}$

\begin{tabular}{lrr}
\hline$[\boldsymbol{g}]$ & $\mathbf{1 A}$ & $\mathbf{2 A}$ \\
\hline $\boldsymbol{n}_{\boldsymbol{g}} \mid \boldsymbol{h}_{\boldsymbol{g}}$ & $\mathbf{1 | 1}$ & $\mathbf{1 | \mathbf { 4 }}$ \\
\hline $\bar{\chi}_{g}^{X_{A}}$ & 1 & 1 \\
$\chi_{g}^{X_{A}}$ & 1 & -1 \\
\hline $\bar{\Pi}_{g}^{X_{A}}$ & $1^{1}$ & $1^{1}$ \\
$\widetilde{\Pi}_{g}^{X_{A}}$ & $1^{24}$ & $2^{12}$ \\
\hline
\end{tabular}

Table 44 Twisted Euler characters and Frame shapes at $\ll=30+6,10,15, X=E_{8}^{3}$

\begin{tabular}{lrrr}
\hline$[\boldsymbol{g}]$ & $\mathbf{1 A}$ & $\mathbf{2 A}$ & $\mathbf{3 A}$ \\
\hline $\boldsymbol{n}_{\boldsymbol{g}} \mid \boldsymbol{h}_{\boldsymbol{g}}$ & $\mathbf{1 | 1}$ & $\mathbf{2} \mid \mathbf{1}$ & $\mathbf{3 | 3}$ \\
\hline $\bar{\chi}_{g}^{X_{E}}$ & 3 & 1 & 0 \\
\hline $\bar{\Pi}_{g}^{X_{E}}$ & $1^{3}$ & $1^{1} 2^{1}$ & $3^{1}$ \\
$\widetilde{\Pi}_{g}^{X_{E}}$ & $1^{24}$ & $1^{8} 2^{8}$ & $3^{8}$ \\
\hline
\end{tabular}




\section{Additional files}

\section{Additional file 1: Coefficient tables.}

Additional file 2: Decompositions.

\section{Acknowledgements}

We owe particular thanks to George Glauberman for alerting us to the connection between the umbral groups of our previous paper and the stabilisers of deep holes in the Leech lattice. This observation was a crucial catalyst for the present work. We also thank Daniel Allcock, Kathrin Bringmann, Scott Carnahan, Noam Elkies, Igor Frenkel, Terry Gannon, Ching Hung Lam, Geoffrey Mason, Atsushi Matsuo, Sameer Murthy, Ken Ono, Erik Verlinde and Don Zagier for helpful comments and discussions. We thank the referees for numerous helpful suggestions and comments. MC would like to thank the Simons Center for Geometry and Physics, Stanford University and the Haussdorff Center for Mathematics for hospitality. The work of JH was supported by NSF grant 1214409.

\section{Author details}

${ }^{1}$ Korteweg-de Vries Institute of Mathematics, University of Amsterdam, the Netherlands. ${ }^{2}$ Department of Mathematics, Applied Mathematics and Statistics, Case Western Reserve University, Cleveland, OH 44106, USA. ${ }^{3}$ Enrico Fermi Institute and Department of Physics, University of Chicago, Chicago, IL 60637, USA.

Received: 21 January 2014 Accepted: 3 February 2014

Published: 17 June 2014

\section{References}

1. Cheng, MCN, Duncan, JFR, Harvey, JA: Umbral moonshine (2012). 1204.2779

2. Niemeier, H-V: Definite quadratische Formen der Dimension 24 und Diskriminante 1. J. Number Theory. 5, 142-178 (1973)

3. Leech, J: Notes on sphere packings. Canad. J. Math. 19, 251-267 (1967)

4. Thompson, JG: Finite groups and modular functions. Bull. London Math. Soc. 11(3), 347-351 (1979)

5. Conway, JH, Norton, SP: Monstrous moonshine. Bull. London Math. Soc. 11, 308-339 (1979)

6. Gannon, T: Moonshine beyond the monster. In: The bridge connecting algebra, modular forms and physics. Cambridge University Press, Cambridge, (2006)

7. Eguchi, T, Taormina, A: Unitary representations of the $N=4$ superconformal algebra. Phys. Lett. B. 196(1), 75-81 (1987). doi:10.1016/0370-2693(87)91679-0

8. Eguchi, T, Taormina, A: Character formulas for the $N=4$ superconformal algebra. Phys. Lett. B. 200(3), 315-322 (1988). doi:10.1016/0370-2693(88)90778-2

9. Eguchi, T, Ooguri, H, Taormina, A, Yang, S-K: Superconformal algebras and string compactification on manifolds with SU(N) holonomy. Nucl. Phys. B315, 193 (1989). doi:10.1016/0550-3213(89)90454-9

10. Eguchi, T, Hikami, K: Superconformal algebras and mock theta functions. J. Phys. A. 42, 3040102009 (2008). 0812.1151

11. Eguchi, T, Ooguri, H, Tachikawa, Y: Notes on the K3 surface and the Mathieu group M24. Exper. Math. 20, 91-96 (2011). 1004.0956

12. Cheng, MCN: $K 3$ surfaces, $N=4$ Dyons, and the Mathieu group $M_{24}$ (2010). 1005.5415

13. Gaberdiel, MR, Hohenegger, S, Volpato, R: Mathieu twining characters for K3. JHEP. 1009, 058 (2010). doi:10.1007/JHEP09(2010)058, 1006.0221

14. Gaberdiel, MR, Hohenegger, S, Volpato, R: Mathieu moonshine in the elliptic genus of K3. JHEP. 1010, 062 (2010). doi:10.1007/JHEP10(2010)062. 1008.3778

15. Eguchi, T, Hikami, K: Note on twisted elliptic genus of K3 surface. Phys. Lett. B694, 446-455 (2011). doi:10.1016/j.physletb.2010.10.017. 1008.4924

16. Gannon, T. Much ado about Mathieu (2012). 1211.5531

17. Cheng, MCN: The largest Mathieu group and (mock) automorphic forms (2012). 1201.4140

18. Gaberdiel, MR: Volpato, R (2012). 1206.5143

19. Creutzig, T, Hoehn, G: The McKay-Thompson series of Mathieu moonshine modulo two (2013). 1211.3703

20. Gaberdiel, MR, Persson, D, Ronellenfitsch, H, Volpato, R: Generalised Mathieu moonshine (2012). 1211.7074

21. Gaberdiel, MR, Persson, D, Volpato, R: Generalised moonshine and holomorphic orbifolds (2013). 1302.5425

22. Taormina, A, Wendland, K: Symmetry-surfing the moduli space of Kummer K3's (2013). 1303.2931

23. Taormina, A, Wendland, $\mathrm{K}$ : A twist in the $M_{24}$ moonshine story (2013). 1303.3221

24. Cappelli, A, Itzykson, C, Zuber, JB: The ADE classification of minimal and $A_{1}^{(1)}$ conformal invariant theories. Commun. Math. Phys. 113, 1 (1987). doi:10.1007/BF01221394

25. Bourbaki, N: Lie groups and lie algebras. Chapters 4-6. Elements of Mathematics (Berlin), p. 300. Springer, Berlin (2002). Translated from the 1968 French original by Andrew Pressley

26. Humphreys, JE: Introduction to Lie Algebras and Representation Theory. Graduate Texts in Mathematics, vol. 9, p. 169. Springer, New York (1972)

27. Conway, JH: A characterisation of Leech's lattice. Invent. Math. 7 (1969)

28. Leech, J: Some sphere packings in higher space. Canad. J. Math. 16, 657-682 (1964)

29. Conway, JH, Parker, RA, Sloane, NJA: The covering radius of the Leech lattice. Proc. Roy. Soc. London Ser. A. 380(1779), 261-290 (1982). doi:10.1098/rspa.1982.0042

30. Conway, JH, Sloane, NJA: Twenty-three constructions for the Leech lattice. Proc. Roy. Soc. London Ser. A. 381(1781), 275-283 (1982). doi:10.1098/rspa.1982.007 
31. Shimura, G: Introduction to the Arithmetic Theory of Automorphic Functions, p. 267. Publications of the Mathematical Society of Japan, No. 11. Iwanami Shoten, Publishers, Tokyo (1971). Kanô Memorial Lectures, No. 1

32. Ferenbaugh, CR: The genus-zero problem for $n \mid h$-type groups. Duke Math. J. 72(1), 31-63 (1993)

33. Conway, JH, Curtis, RT, Norton, SP, Parker, RA, Wilson, RA: Atlas of finite groups. Maximal subgroups and ordinary characters for simple groups. With Comput. Assist. from J. G. Thackray. Clarendon Press, Oxford (1985)

34. McKay, J: Graphs, singularities, and finite groups. In: The Santa Cruz Conference on Finite Groups (Univ. California, Santa Cruz, Calif., 1979). Proc. Sympos. Pure Math. vol. 37, pp. 183-186. Amer. Math. Soc. Providence (1980)

35. Slodowy, P: Simple Singularities and Simple Algebraic Groups. Lecture Notes in Mathematics, vol. 815, p. 175. Springer, Berlin (1980)

36. Gonzalez-Sprinberg, G, Verdier, J-L: Construction géométrique de la correspondance de McKay. Ann. Sci. École Norm. Sup. (4). 16(3), 409-4491984 (1983)

37. Bruinier, JH, Funke, J: On two geometric theta lifts. Duke Math. J. 125(1), 45-90 (2004). doi:10.1215/S0012-7094-04-12513-8

38. Zwegers, S: Mock theta functions. PhD thesis, Utrecht University (2002)

39. Zagier, D: Ramanujan's mock theta functions and their applications (after Zwegers and Ono-Bringmann). Astérisque. 326, 986-1431642010 (2009). Séminaire Bourbaki. Vol. 2007/2008

40. Eichler, M, Zagier, D: The theory of Jacobi forms. Birkhäuser, Cambridge (1985)

41. Dabholkar, A, Murthy, S, Zagier, D: Quantum black holes, wall crossing and mock modular forms (2012). 1208.4074

42. Shimura, G: On modular forms of half integral weight. Ann. Math. (2). 97, 440-481 (1973)

43. Gepner, D, Qiu, Z-a: Modular invariant partition functions for parafermionic field theories. Nucl. Phys. B285, 423 (1987). doi:10.1016/0550-3213(87)90348-8

44. Skoruppa, N-P, Zagier, D: Jacobi forms and a certain space of modular forms. Invent. Math. 94(1), 113-146 (1988). doi:10.1007/BF01394347

45. Skoruppa, N-P: Explicit formulas for the Fourier coefficients of Jacobi and elliptic modular forms. Invent. Math. 102(3), 501-520 (1990). doi:10.1007/BF01233438

46. Skoruppa, N-P: Heegner cycles, modular forms and Jacobi forms. Sém. Théor. Nombres Bordeaux (2). 3(1), 93-116 (1991)

47. Hoehn, G: Selbstduale Vertexoperatorsuperalgebren und das Babymonster (Self-dual Vertex Operator Super Algebras and the Baby Monster). Bonner Mathematische Schriften. 286, 1-851996 (2007). 0706.0236

48. Witten, E: Three-dimensional gravity revisited (2007). 0706.3359

49. Skoruppa, N-P: Über Den Zusammenhang Zwischen Jacobiformen und Modulformen Halbganzen Gewichts. Bonner Mathematische Schriften [Bonn Mathematical Publications], 159, p. 163. Universität Bonn Mathematisches Institut, Bonn (1985). Dissertation, Rheinische Friedrich-Wilhelms-Universität, Bonn, 1984

50. Ramanujan, S: The Lost Notebook and Other Unpublished Papers, p. 419. Springer, Berlin (1988). With an introduction by George E. Andrews

51. Gordon, B, Mclntosh, RJ: Some eighth order mock theta functions. J. London Math. Soc. (2). 62(2), 321-335 (2000). doi:10.1112/S0024610700008735

52. McIntosh, RJ: Second order mock theta functions. Canad. Math. Bull. 50(2), 284-290 (2007). doi:10.4153/CMB-2007-028-9

53. Cheng, MCN, Duncan, JFR: On Rademacher sums, the largest Mathieu group and the holographic modularity of moonshine (2011). 1110.3859

54. Cheng, MCN, Duncan, JFR: On the discrete groups of Mathieu moonshine. ArXiv e-prints (2012). 1212.0906

55. Benard, M: Schur indexes of sporadic simple groups. J. Algebra. 58(2), 508-522 (1979). doi:10.1016/0021-8693(79)90177-7

56. Margolin, RS: Representations of M12. J. Algebra. 156(2), 362-369 (1993). doi:10.1006/jabr.1993.1078

57. Creutzig, T, Hoehn, G, Miezaki, T: The McKay-Thompson series of Mathieu moonshine modulo two. ArXiv e-prints (2012). 1211.3703

58. Dijkgraaf, R, Verlinde, E, Verlinde, H: Counting Dyons in $\mathrm{N}=4$ string theory. Nucl. Phys. B. 484, 543-561 (1997). (Nucl.Phys.B484:543-561, 1997). hep-th/9607026

59. Kawai, T: $N=2$ heterotic string threshold correction, K3 surface and generalized Kac-Moody superalgebra. Phys. Lett. B372, 59-64 (1996). doi:10.1016/0370-2693(96)00052-4. hep-th/9512046

60. Lopes Cardoso, G, Curio, G, Lust, D: Perturbative couplings and modular forms in $\mathrm{N}=2$ string models with a Wilson line. Nucl.Phys. B491, 147-183. doi:10.1016/S0550-3213(97)00047-3. hep-th/9608154

61. Raum, M: $\mathbf{M}_{24}$-twisted product expansions are Siegel modular forms (2012). 1208.3453

62. Gritsenko, VA, Nikulin, W: K3 surfaces, Lorentzian Kac-Moody algebras and mirror symmetry. Math. Res. Lett. 3(2), 211-229 (1996)

63. Gritsenko, VA, Nikulin, W: The arithmetic mirror symmetry and Calabi-Yau manifolds. Comm. Math. Phys. 210(1), 1-11 (2000). doi:10.1007/s002200050769

64. Gritsenko, VA, Nikulin, W: Automorphic forms and Lorentzian Kac-Moody algebras. I. Internat. J. Math. 9(2), 153-199 (1998). doi:10.1142/S0129167X98000105

65. Gritsenko, VA, Nikulin, W: Automorphic forms and Lorentzian Kac-Moody algebras. II. Internat. J. Math. 9(2), 201-275 (1998). doi:10.1142/S0129167X98000117

66. Gritsenko, VA, Nikulin, W: Siegel automorphic form corrections of some Lorentzian Kac-Moody Lie algebras. Amer. J. Math. 119(1), 181-224 (1997)

67. Kondo, S: Niemeier lattices, Mathieu groups, and finite groups of symplectic automorphisms of $K 3$ surfaces. With an appendix by Shigeru Mukai. Duke Math. J. 92(3), 593-603 (1998)

68. Nikulin, W: Kählerian K3 surfaces and Niemeier lattices, I (2011). 1109.2879

69. Cheng, MCN, Dong, X, Duncan, J, Harvey, J, Kachru, S, et al.: Mathieu moonshine and N = 2 string compactifications (2013). 1306.4981 
70. Aoki, $\mathrm{H}$, Ibukiyama, $\mathrm{T}$ : Simple graded rings of Siegel modular forms, differential operators and Borcherds products. Int. J. Math. 16 No. 3, 249-279 (2005)

71. Gritsenko, V: Elliptic genus of Calabi-Yau manifolds and Jacobi and Siegel modular forms. Algebra i Analiz. 11(5), 100-125 (1999)

72. The GAP Group: GAP - Groups, Algorithms, and Programming, Version 4.4. (2005). The GAP Group http://www.gap-system.org

doi:10.1186/2197-9847-1-3

Cite this article as: Cheng et al:: Umbral moonshine and the Niemeier lattices. Research in the Mathematical Sciences 2014 1:3.

Submit your manuscript to a SpringerOpen ${ }^{\circ}$ journal and benefit from:

- Convenient online submission

- Rigorous peer review

- Immediate publication on acceptance

- Open access: articles freely available online

- High visibility within the field

- Retaining the copyright to your article

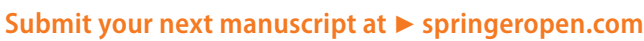

